\title{
Mid-IR emission of galaxies in the Virgo cluster III. The data ${ }^{\star}$
}

\author{
A. Boselli ${ }^{1}$, M. Sauvage ${ }^{2}$, J. Lequeux ${ }^{3}$, A. Donati ${ }^{4}$, and G. Gavazzi ${ }^{4}$ \\ ${ }^{1}$ Laboratoire d'Astrophysique de Marseille, BP 8, Traverse du Siphon, 13376 Marseille Cedex 12, France \\ e-mail: Alessandro.Boselli@oamp.fr \\ 2 DSM/DAPNIA/Service d'Astrophysique, CEA/Saclay, 91191 Gif-sur-Yvette, France \\ e-mail: msauvage@cea.fr \\ 3 DEMIRM and URA 336 du CNRS, Observatoire de Paris, 61 Av. de l'Observatoire, 75014 Paris, France \\ e-mail: James.Lequeux@obspm. fr \\ ${ }^{4}$ Università degli Studi di Milano-Bicocca, Dipartimento di Fisica, Piazza dell'Ateneo Nuovo 1, 20126 Milano, Italy \\ e-mail: Alessandro.Donati@mib.infn.it;Giuseppe.Gavazzi@mib.infn.it
}

Received 1 October 2002 / Accepted 16 May 2003

\begin{abstract}
We present ISOCAM imaging data at 6.75 and $15 \mu \mathrm{m}$ for 145 galaxies in the Virgo cluster and in the Coma/A1367 supercluster. Of these, 99 form a complete, optically-selected, volume-limited sample including galaxies in the core and in the periphery of the Virgo cluster, suitable for statistical analysis. 34 of the Virgo galaxies were resolved by ISOCAM: for these objects we present mid-IR images, radial light and colour profiles, as well as effective and isophotal radii, surface brightness and light concentration indices.

The mid-IR colours of the target galaxies show a weak trend with the $H$ band luminosity, with values of $F(6.75 \mu \mathrm{m}) / F(15 \mu \mathrm{m}) \geq$ 1 found generally in massive objects $\left(L_{H} \geq 10^{10} L_{H \odot}\right)$, and $F(6.75 \mu \mathrm{m}) / F(15 \mu \mathrm{m}) \leq 1$ in low-mass $\left(L_{H} \leq 10^{10} L_{H \odot}\right)$ dwarf galaxies. All early-type galaxies (type $\leq \mathrm{S} 0$ a) have $F(6.75 \mu \mathrm{m}) / F(15 \mu \mathrm{m}) \geq 1$, as expected when the mid-IR emission is dominated by the photosphere of the cold stellar population. The mid-IR, near-IR and visible light concentration indices of bright galaxies are tightly correlated with one another, indicating that the spatial distribution of the mid-IR emitting sources is, to the first order, similar to that of the stars.
\end{abstract}

Key words. galaxies: general - galaxies: spiral - galaxies: ISM - stars: formation

\section{Introduction}

Interstellar dust plays an important role in the energy balance of galaxies. Dust grains efficiently absorb the stellar light and re-emit the absorbed energy in the infrared. A large fraction of the energy emitted by a galaxy therefore lies in the infrared, ranging from $\sim 30 \%$ in normal galaxies such as the Milky Way up to more than $90 \%$ in ultra-luminous infrared galaxies (Cox \& Mezger 1989).

Observations with the IRAS, COBE and ISO satellites have demonstrated the existence of dust grains with a large range of sizes, from big grains (with a radius $a \gtrsim 200 \AA$ ) to very small grains $(10 \AA \lesssim a \lesssim 200 \AA$ ) and even smaller particles responsible for the Aromatic Infrared Bands (AIBs). Depending on the radiation field, these grains are or are not in thermal equilibrium. For example, in the radiation field near the Sun which is typical for an ordinary spiral galaxy, the big grains which contain most of the dust mass are in thermal equilibrium with a

Send offprint requests to: A. Boselli, e-mail: Alessandro.Boselli@oamp.fr

* Figures 1 and 2, and Tables 1, 2, 4, 5 are only available in electronic form at http://www. edpsciences.org typical temperature of $17.7 \mathrm{~K}$ (Lagache et al. 1999). However, there are also colder grains with temperatures as low as $\approx 12 \mathrm{~K}$ in regions shielded from visible and UV radiation (Ristorcelli et al. 1999). At the other extreme of the grain size range, the carriers of the AIBs are always heated stochastically by absorption of single photons and temporarily reach very high temperatures, at which most of the emission occurs (Draine \& Li 2001). The situation with the intermediate very small grains is more complex, because the size of grains that exhibit stochastic heating and of those that emit at equilibrium temperature depends on the radiation field.

The intensity emitted at frequency $v$ by grains at temperature $T$ is $I_{v}=\tau B_{v}(T)$, where $B_{v}(T)$ is the specific intensity of the blackbody at temperature $T$ and the optical depth $\tau$ is roughly proportional to $v^{-2}$. Consequently, the peak of emission for cold grains $(12-20 \mathrm{~K})$ is at wavelengths longer than $100 \mu \mathrm{m}$. Stochastically heated grains reach temporarily high temperatures, at which most of the energy is radiated, so that the peak of their emission is at much shorter wavelengths. Models for the optical properties of grains consistent with the local dust extinction and emission properties of the Milky Way have been presented in Li \& Draine (2001). 
Such dust models can be used as the basis for self-consistent calculations of grain heating, incorporating realistic geometries, in well-studied late-type galaxies. Such calculations show for example that at $60 \mu \mathrm{m}$, most of the integrated emission of a normal galaxy is not dominated by very small grains, as is the case for the emission from grains immersed in the interstellar radiation field near the Sun (Dsert et al. 1990; Dwek et al. 1997), but mainly (about 60\%) by bigger grains in equilibrium with the high radiation fields in the star-forming regions: see e.g. Fig. 8 and Table 3 of Popescu et al. (2000) for the case of NGC 891. At this wavelength, only about $20 \%$ of the emission comes from stochastically heated grains in the outer disks, with approximately another $20 \%$ arising from big grains in equilibrium with the diffuse optical photons in the central regions of galaxies.

There is another difficulty, of observational nature, in the interpretation of far-infrared observations of galaxies. The cold grains, which contain most of the mass of the dust, could barely be seen in the $100 \mu \mathrm{m}$ filter of IRAS, which therefore offered a biased view of dust emission in galaxies. ISO extended the observing wavelength range to $200 \mu \mathrm{m}$, providing a better insight on this emission and consequently enabling a more complete knowledge of dust emission and energy balance in galaxies (Popescu et al. 2002; Popescu \& Tuffs 2002).

To which extent the mid- and far-IR emission can be used as a tracer of star formation in galaxies is still unclear. The farinfrared wavelength range is more favorable a priori, since it carries the bulk of the luminosity re-radiated by grains, most of which arise from the young stellar population. Whether the mid-IR can also be of use is an interesting and still open question, which could resolved by statistical comparisons of mid-IR data with star formation rates corrected for extinction and derived from a combination of UV/optical and farIR/submillimeter data. It should be reminded at this stage that the spectral energy distribution of the mid-IR emission in the UIBs and associated continuum does not depend on the intensity of the radiation field, but only on its hardness. This might ease the interpretation of the corresponding observations.

With this aim in mind amongst others, the ISOCAM and ISOPHOT consortia defined a coordinated program of observations of an optically selected, complete sample of late type galaxies, which was executed in the guaranteed time of the ISOCAM and ISOPHOT instrument teams. The sample of Virgo cluster galaxies was chosen because, at that time, it was the deepest complete sample available of normal star-forming galaxies. Most of these galaxies are thought to be freshly falling in from the field, before being affected by the cluster environment. However the sample contains galaxies in the central region that are clearly affected. Roussel et al. (2001b) have presented ISOCAM data for 23 of the brightest Virgo galaxies which add to the present sample. The Virgo sample can be compared to the Coma/A1367 supercluster ISO sample (see later), which is more affected by the environment, and to the samples of nearby field galaxy of the ISO surveys of Dale et al. (2000) and of Bendo et al. (2002).

In this paper we report on the ISOCAM consortium observations at 6.75 and $15 \mu \mathrm{m}$ and present the results of new reductions made using updated CIA data reduction tools, i.e. $\mathrm{v}$ 4.0. We discuss and analyze the mid-IR properties of late-type galaxies. The PHOT and LWS data are presented in Tuffs et al. (2002) and Leech et al. (1999) respectively, and discussed respectively by Popescu et al. (2002), Pierini et al. $(1999,2001)$ and Boselli et al. $(2002 ; 2003)$. The origin of the dust emission of late-type galaxies and its relation with star formation, already presented in Boselli et al. (1997a, 1998), will be discussed further in future papers. We also present homogeneously reduced mid-IR data for other galaxies in the Virgo cluster and in the Coma/A1367 supercluster. The total number of galaxies we discuss is 145 . It represents, up to date, the largest available sample in the literature with ISOCAM data.

The samples are presented in Sect. 2, the observations, the data reduction procedures and the image analysis in Sects. 3 and 4 . The Mid-IR properties of 34 spatially resolved galaxies are given in Sect. 4. The data are compared to those available in the literature in Sect. 5, while a simple statistical analysis of the mid-IR properties of late-type galaxies is given in Sect. 6.

\section{The sample}

The present paper reports ISOCAM observations of 123 galaxies in the Virgo cluster region $\left(12^{\mathrm{h}} \leq \mathrm{RA} \leq 13^{\mathrm{h}}, 0^{\circ} \leq \mathrm{Dec} \leq\right.$ $18^{\circ}$ ) and 22 objects in the Coma/A1367 supercluster region $\left(11^{\mathrm{h}} 30^{\mathrm{m}} \leq \mathrm{RA} \leq 13^{\mathrm{h}} 30^{\mathrm{m}}, 18^{\circ} \leq \operatorname{Dec} \leq 32^{\circ}\right)$.

The present sample can be subdivided in 5 different subsamples, defined as:

- The optically-selected, volume-limited, complete ISO Virgo sample, defined as described in Boselli et al. (1997b, 1998), observed in guaranteed time as part of the Virgo central program with CAM, PHOT ${ }^{1}$ (Tuffs et al. 2002) and partly with LWS $^{2}$ (Leech et al. 1999). This sample has been extracted from the Virgo Cluster Catalogue (VCC) of Binggeli et al. (1985), which is complete to $B_{\mathrm{T}}=18$, to include all late-type galaxies (type $\geq \mathrm{S} 0$ a) with $B_{\mathrm{T}}<18$, classified as cluster member by Binggeli et al. $(1985,1993)$. For telescope time limitation, out of the 207 VCC galaxies matching these criteria, only 100 were selected. These are objects with projected separation $<2^{\circ}$ from M 87 (cluster core) or at the periphery of the cluster, with an angular separations greater than $4^{\circ}$ from the position of maximum projected galaxy density given by Sandage et al. (1985), but excluding galaxies within $1.5^{\circ}$ of the M 49 sub-cluster. To limit the spread of distances within the sample, galaxies belonging to the $\mathrm{M}$ and $\mathrm{W}$ clouds (as defined by Binggeli et al. 1985, 1993), and in the Southern extension $\left(\delta<5^{\circ}\right)$ were also excluded.

The total sample includes 118 objects (see Boselli et al. 2003). Because of the low visibility of the Virgo cluster during the ISO mission and because of the low detection rate

\footnotetext{
1 Sixty-one out of the 100 ISO Virgo sample galaxies (see Sect. 2) were observed by ISOPHOT. These comprise all galaxies west from RA(1950) $12.596 \mathrm{~h}$ with $B_{\mathrm{T}}$ brighter than $16.8 \mathrm{mag}$ in the cluster periphery and $B_{\mathrm{T}}$ brighter than $14.5 \mathrm{mag}$ in the cluster core areas.

2 Eighteen out of the 100 ISO Virgo sample galaxies were observed by ISOLWS. These comprise all objects with type $\leq \operatorname{Scd}$ and $B_{\mathrm{T}} \leq$ 12.3, with the exception of the interacting system VCC 1673-1676 and VCC 836.
} 
of small galaxies with ISOCAM, the $18 \mathrm{Im}$ and BCDs in the cluster core could not be observed. The resulting sample is still unbiased but includes only 100 objects, with 28 spirals $(\mathrm{S} 0 / \mathrm{Sa}-\mathrm{Sm})$ in the cluster core, and 72 spirals, irregulars and BCDs in the cluster periphery. It thus constitutes an optically-selected, volume-limited complete sample, ideal for statistical analyses. 99 out of the 100 galaxies were observed by ISOCAM ${ }^{3}$. The sample includes galaxies spanning the whole range in morphological type for late-type systems, from S0/a to Im and BCDs, luminosity $\left(-21 \leq M_{B} \leq-13\right)$, and galaxy density (cluster core vs. cluster periphery).

- The Virgo serendipitous sample is composed of 18 galaxies with optical counterparts in the VCC, serendipitously observed in the field of the ISO Virgo galaxies. This sample is dominated by early-type systems, mostly dwarf ellipticals (10 objects). Despite its small dimension, this subsample can be taken as representative of a mid-IR selected sample of nearby galaxies. Two of the 18 objects (VCC 899 and VCC 1018) are background galaxies.

- Six galaxies belonging to the Virgo cluster were observed with ISOCAM as part of other guaranteed or open time proposals. Out of the 6 objects, only one is not included in the VCC. The ISOCAM data, now available at the ISO data centre, are here reduced, analyzed and presented as homogeneously as possible with those of the ISO Virgo sample providing to the community the largest available dataset of galaxies in the Virgo cluster region. Since the observations of these objects were taken for different purposes, the photometric quality of the present data is not always as good as that of the ISO Virgo sample.

- The A1367 and Coma clusters sample, extensively described in Contursi et al. (2001), includes 19 late-type galaxies. Briefly, the sample is composed of high star forming galaxies with peculiar morphologies, blue colour indices $(B-H \leq 2.75)$, characterized by asymmetric HI and/or $\mathrm{H} \alpha$ distributions or indicating any possible sign of an ongoing interaction with the intracluster medium. The present sample is thus not complete in any sense. The CAM data, already presented in Contursi et al. (2001), are here reprocessed using the new data reduction tools and are thus directly comparable to those of the Virgo samples. Data for the serendipitously detected CGCG97-087N are added.

- We present CAM data of other 3 galaxies in the Coma/A1367 supercluster region; two of them (CGCG159072) form an interacting system, known as "The mices" (Arp 242), the third one (CGCG130-023) is a galaxy in the small group of UGC 8248 (Gavazzi et al. 1999b).

Table 1 gathers the principal information on the target galaxies, arranged as follows:

- Column 1: VCC denomination for the Virgo cluster galaxies (Binggeli et al. 1985, hereafter VCC), CGCG for the objects in the Coma/A1367 supercluster (Zwicky et al. 19611968, hereafter CGCG).

3 The galaxy VCC 1869, unfortunately not observed by ISOCAM, should be added to these 99 objects to form the exact complete sample.
- Column 2: UGC name (Nilson 1973).

- Column 3: NGC or IC name.

- Columns 4 and 5: (J2000.0) celestial coordinates with few arcsec accuracy, from the VCC, or measured by us on the DSS.

- Column 6: morphological type, from the VCC or from Binggeli et al. (1993) for Virgo galaxies, from Gavazzi \& Boselli (1996) for the other objects.

- Column 7: photographic magnitude from the VCC for the Virgo galaxies, from the CGCG for the other objects.

- Columns 8 and 9: major $(a)$ and minor $(b)$ optical diameters (arcmin) determined at the surface brightness of 25 th $\mathrm{mag} \operatorname{arcsec}^{-2}$. From the UGC (when available) or consistently determined by us. For VCC galaxies the diameter is computed from the "last visible" isophotal diameter given in the VCC using: $\log a_{\text {ext }}\left(b_{\text {ext }}\right)=0.99 \log a(b)+0.1$.

- Column 10: distance, in Mpc. Distances to the various substructures of Virgo are as given in Gavazzi et al. (1999a). A distance of 96 and $91.3 \mathrm{Mpc}$ is assumed for galaxies in Coma and A1367 respectively. For galaxies not belonging to the clusters, the distance is determined from the redshift assuming $H_{0}=75 \mathrm{~km} \mathrm{~s}^{-1} \mathrm{Mpc}^{-1}$.

- Column 11: cluster membership as defined in Gavazzi et al. (1999a) for Virgo and in Gavazzi et al. (1999b) for the Coma/A1367 supercluster ${ }^{4}$. Codes A, B, M, W, S, N and $\mathrm{E}$ are for clusters $\mathrm{A}$ and $\mathrm{B}, \mathrm{M}$ and $\mathrm{W}$ clouds, southern extension, $\mathrm{N}$ and $\mathrm{E}$ substructures respectively.

- Column 12: projected angular distance from the cluster centre (M 87), in degrees. Among the ISO Virgo galaxies, the core subsample is composed by all objects with $\theta<2$ degrees.

- Columns 13 and 14: the $K$ and $B$ band $C_{31}(K)$ and $C_{31}(B)$ concentration index, defined as the model independent ratio between the radii that enclose $75 \%$ and $25 \%$ of the total light, from Gavazzi et al. (2000) and Gavazzi et al., in preparation.

- Column 15: notes on morphological type, from Boselli et al. (2003) for the ISO Virgo sample, from NED for other objects, unless specified.

\section{Observations and data reduction}

All galaxies were observed in 2 filters, $\operatorname{LW} 2(\lambda=6.75 \mu \mathrm{m}$, $\Delta \lambda=5.00-8.50 \mu \mathrm{m})$ and LW3 $(\lambda=15.0 \mu \mathrm{m}, \Delta \lambda=$ $12.0-18.0 \mu \mathrm{m})$.

Observations of the ISO Virgo sample were part of the ISOCAM Central Program: all galaxies were observed in one of the few visibility windows of the Virgo cluster during the ISO mission, in the summer of 1996 . The $32 \times 32$ pixel longwavelength camera was used to make a raster map covering the whole pointed galaxy and its nearby background, with a shift (then overlap) of 16 pixels between adjacent positions.

${ }^{4}$ The Gavazzi et al. (1999a) cluster membership criterion, based on the analysis of the 3-D distribution of galaxies in the Virgo cluster, is slightly different from that used by Binggeli et al. $(1985,1993)$. It is thus not surprising that several objects in Table 1 appear as members of cluster B or of the M and W clouds. 
The pixel size was $6^{\prime \prime} \times 6^{\prime \prime}$, with a diffraction limit of $6 \operatorname{arcsec}$ at $6.75 \mu \mathrm{m}$ and of $8 \operatorname{arcsec}$ at $15 \mu \mathrm{m} .3 \times 3$ rasters were made for galaxies with the smallest angular dimensions, while larger rasters, up to $8 \times 8$, were adopted for giant or extended objects. The extension of the combined map was selected to exceed by a factor of $\sim 2-3$ the optical angular dimension of the galaxy in order to allow an accurate determination of the background contribution. All rasters were performed along the satellite axes, which are aligned with the camera axes. This implies that the orientation of the North axis with respect to the raster axes is in principle different from one map to the other. This orientation is listed in Table 4, where the quoted angle is from the North axis to the main scanning axis of the raster (i.e. that on which ghosts due to transients appear). As can be seen there is in fact little dispersion because the observations were performed in a small group of nearby orbits. Table 5 also lists the ISO TDT number which can be useful to locate our observations in the ISO archive ${ }^{5}$. The elementary integration time was $2.1 \mathrm{~s}$, with 16 to 20 integrations per position. To minimize the effects of transients of the detector on the determination of the source flux, 40 to 70 integrations per position were used in the first raster position every time the background was expected to change significantly from the previous target, i.e. for significantly different pointings and for different filters.

The large raster maps allowed us to observe other Virgo galaxies: only those fully covered by the CAM image were included in the Virgo serendipitous sample.

An accurate description of the observations of the A1367 and Coma clusters galaxies is given in Contursi et al. (2001). This program was prepared to follow most of the procedures used in the Virgo galaxy observations. Given the small angular size of the targets, images were obtained by mosaics of $2 \times 2$ raster positions with shifts of 10 pixels $\left(60^{\prime \prime}\right)$ between successive positions. The final images have sizes of $42 \times 42$ pixels, or $4.2^{\prime} \times 4.2^{\prime}$. The central $22 \times 22$ pixels, containing the galaxy, were sampled 4 times. All observations were done with an elementary integration time of $5.04 \mathrm{~s}$, with $\sim 10$ integrations per position, 20 for the first raster position.

In order to minimize the overhead time for galaxy pointing and for the stabilization of the detector, galaxies in the ISO Virgo sample as well as in the clusters A1367 and Coma were observed in a concatenated mode, with a complete sequence of observations of 5-10 nearby galaxies in a given (and fixed) filter.

The remaining 6 galaxies in the Virgo cluster as well as the 3 objects in the Coma/A1367 supercluster were part of different and thus heterogeneous observing programs. In particular, only the central region of the three early-type objects in Virgo was mapped, making the determination of the sky emission, and thus of the total flux density, highly uncertain.

Images were reduced following the standard $\mathrm{CIA}^{6}$ procedures available at the ISO center at CEA/Saclay: images

\footnotetext{
${ }^{5}$ http://www.iso.vilspa.esa.es/

${ }^{6}$ CAM Interactive Analysis, CIA, is a joint development by the ESA Astrophysics Division, and the ISOCAM Consortium led by the ISOCAM P.I., C. Cesarsky, Direction des Sciences de la Matière, CEA, France.
}

were dark-subtracted using a time-dependent model of the dark current (Siebenmorgen et al. 2000), then deglitched using the multi-resolution method described in Starck et al. (1999). Data cubes were then transient-corrected using the algorithm developed by Coulais \& Abergel (2000). It is well known that even with this transient correction, there remains in the ISOCAM data a long term drift, called "long-term transient" by Miville-Deschênes et al. (2000) which manifests itself as an increase of the background level as the raster progresses. We have used the methods developed by Miville-Deschênes et al. (2000) to correct for this long-term transient, as well as to flatfield the data, the two corrections being intimately linked. The routines necessary for these corrections are available at CIA, through the SLICE package. Even with these, we often observed a 2-D gradient in the background of the final, reduced images. This gradient was removed using the task IMSURFIT in IRAF/IMAGES ${ }^{7}$. In Sect. 4.1 we come back to the uncertainties introduced by the data reduction process.

The absolute astrometric accuracy of the reconstructed rasters is dominated by the lens wheel jitter which introduces an absolute uncertainty of 1.5-2 pixels, i.e. 9-12". The relative pointing uncertainty, i.e. that which relates to successive pointings inside a raster, is much smaller, at $0 .{ }^{\prime \prime} 5$. Given that the observations we made in rather long concatenated chains of rasters, the same absolute astrometric error will apply to the rasters obtained in the same concatenation chain. For the same reason, since the LW2 and LW3 observations were generally made in different chains, a different pointing error occur in the two maps of the same galaxy.

\section{Image analysis}

The total flux densities were extracted using the QPHOT routine in IRAF/NOAO after subtraction of the sky background estimated in a concentric annulus outside the galaxy, of radius $\sim 20 \%$ larger than the mid-IR extension of the galaxy. Total counts have been obtained by integrating the pixel counts over the area covered by each galaxy, as derived by its mid-IR image, i.e. within a circle of radius $r_{\lambda}, \sim 10 \%$ larger than the isophotal radii $r_{5}(6.75)$ and $r_{5}(15)$ as given in Eqs. (5) and (6). Counts were transformed into flux densities using the conversion factors given in the ISOCAM handbook (Siebenmorgen et al. 2000), i.e. by dividing by $2.32 \mathrm{ADU}$ gain ${ }^{-1} \mathrm{~s}^{-1} \mathrm{mJy}^{-1}$ for LW2 and 1.96 ADU gain ${ }^{-1} \mathrm{~s}^{-1} \mathrm{mJy}^{-1}$ for LW3.

For undetected galaxies, an upper limit has been determined as:

$F_{\text {limit }(\lambda)}=2 \sqrt{\left[\sigma(s)_{\lambda}\left(\pi r_{\lambda}^{\prime 2}\right)\right]^{2}+\left[\sigma(p)_{\lambda} \sqrt{\left(\pi r_{\lambda}^{\prime 2}\right)}\right]^{2}}(\mathrm{mJy})$

where $\sigma(p)_{\lambda}$ is the background noise per pixel at a given $\lambda$, $\sigma(s)_{\lambda}$ is the uncertainty on the sky determination and $r_{\lambda}^{\prime}$ is the expected mid-IR radius (in pixel units, 6 arcsec/pixel) of the observed galaxy. $r_{\lambda}^{\prime}$ has been estimated using Eqs. (5) and (6),

\footnotetext{
${ }^{7}$ IRAF is the Image Analysis and Reduction Facility made available to the astronomical community by the National Optical Astronomy Observatories, which are operated by AURA, Inc., under contract with the U.S. National Science Foundation.
} 
determined from the relationship between optical and mid-IR isophotal radii of resolved galaxies (see Sect. 4.2). The upper limit to the flux density corresponds to $2 \times \operatorname{err}_{\lambda}$, where $\operatorname{err}_{\lambda}$ (see Eq. (4)) would be the total error for the flux density if the galaxy was detected (see Sect. 4.1).

The results of the observations are given in Table 2, organized as follows:

- Column 1: galaxy name.

- Column 2: raster map.

- Column 3: total field of view, in arcminutes.

- Column 4: elementary integration time.

- Columns 5, 6, 7, 8 and 9: mid-IR flux density (in mJy) with error, background surface brightness, uncertainty on the sky determination $\sigma(s)_{\lambda}$, pixel noise $\sigma(p)_{\lambda}$ and photometric quality of the data at $6.75 \mu \mathrm{m}$. For galaxies with two independent data reductions (see Sect. 4.1), Col. 5 (and 10) gives the average value. The error is determined using Eq. (4) given in Sect. 4.1. The $\mathrm{rms} \sigma(s)_{\lambda}$ used in the error determination has been computed as the standard deviation on the estimate of the sky background, whose accuracy is limited by the quality of the flat-fielding. This is, on average, a factor of 2 lower than the rms on the single pixel counts $\sigma(p)_{\lambda}$. The background surface brightness, $\sigma(s)_{\lambda}$ and $\sigma(p)_{\lambda}$ are given in units of $\mu \mathrm{Jy} \operatorname{arcsec}^{-2}$. A photometric quality of 1 is for high signal to noise images with a flat sky background. A value of 2 is for low signal to noise data, for images with strong residual gradients in the sky background, for objects where the transient memory of the bright nucleus is not perfectly corrected and partly contaminates the disc emission in the reduced image, or for those objects whose photometric parameters differ by more than $\sim 30 \%$ when images are reduced using different parameters (see Sect. 4.1). In all these objects the photometric uncertainty is expected to be larger than on the average. A photometric quality of 3 is given to tentative detections. These data should be used with caution in any statistical analysis.

- Columns 10, 11, 12, 13 and 14: mid-IR flux density (in $\mathrm{mJy}$ ) with error, background surface brightness, uncertainty on the sky determination $\sigma(s)_{\lambda}$, pixel noise $\sigma(p)_{\lambda}$ and photometric quality of the data at at $15 \mu \mathrm{m}$.

- Column 15: an asterisk indicates notes to individual objects, reported at the end of the table. For the serendipitous Virgo sample, Col. 15 lists the target name of the frame where the galaxy was detected.

The detection rate is $77 \%$ and $67 \%$ at 6.75 and $15 \mu \mathrm{m}$ respectively for the ISO Virgo sample, $28 \%$ and $22 \%$ for the Virgo serendipitous sample, and $100 \%$ at both frequencies for the other samples. The 6.75 and $15 \mu \mathrm{m}$ gray-level images of the fully resolved galaxies (see Sect. 4.2) are given in Fig. 1, while the contour plot of all the other detected galaxies (with a surface brightness brighter than $2 \mu \mathrm{Jy} \operatorname{arcsec}^{-2}$ ) are shown in Fig. 2.

\subsection{Photometric uncertainty}

All the possible origins of photometric errors in ISOCAM images are extensively discussed in Roussel et al. (2001b). Those which are going to affect the present data are mostly related to (1) remnants of transient on the galaxies' nuclei that either decrease the responsivity of the array, or leave ghosts in the map, (2) the use of an incorrect flat-field, (3) an incomplete correction of the long-term transient leading to a wrong assessment of the background. It is not possible to perform statistical tests on point (1) since it would mean changing the parameters of the Coulais \& Abergel (2000) model, thus abandoning the physical basis that is its strength. One can only identify on the images the presence of these ghosts and label the fluxes accordingly (see Table 2). For the other effects, it is possible to investigate their amplitude by adjusting the correction parameters. These parameters are in fact mostly those dealing with long-term transient and flat-field corrections.

Inaccurate corrections for the long-term transient and/or for flat-fielding lead to large uncertainties in the sky background determination. These uncertainties are larger than those induced by the statistical noise of the image on extended sources, where are the major sources of error for the flux determination. Inaccurate corrections for long-term transient and/or flatfielding can introduce 2-D structures on the image background on scale comparable or larger than the angular size of the target galaxies, leading to systematic errors on the sky background. Using the uncertainty on the sky determination $\left(\sigma(s)_{\lambda}\right)$ (Cols. 7 and 12 in Table 2), we can compute the error on the flux density determination due to long-term transient and/or for flatfielding, $\operatorname{err}(s)_{\lambda}$, using the relation:

$\operatorname{err}(s)_{\lambda}=\sigma(s)_{\lambda}\left(\pi r_{\lambda}^{2}\right) \quad(\mathrm{mJy})$

where $r_{\lambda}$ is the radius (in pixels) of the circular aperture inside which the galaxy counts were determined ${ }^{8}$.

For point-like, faint sources near the detection limit the random error due to photon noise from the dominant foreground zodiacal light, $\operatorname{err}(p)_{\lambda}$, can be dominant. Random uncertainties can be estimated using the relation:

$\operatorname{err}(p)_{\lambda}=\sigma(p)_{\lambda} \sqrt{\left(\pi r_{\lambda}^{2}\right)} \quad$ (mJy)

where $\sigma(p)_{\lambda}$ (from Cols. 8 and 13 in Table 2) is the pixel per pixel rms. The total uncertainty in the flux determination (given in Table 2) is thus:

$\operatorname{err}_{\lambda}=\sqrt{\operatorname{err}(s)_{\lambda}^{2}+\operatorname{err}(p)_{\lambda}^{2}} \quad(\mathrm{mJy})$.

Since we did not included the uncertainties induced by transient remnants (see previous point 1), the total error given in Eq. (4) is underestimated.

Indeed, this relation generally leads to photometric errors smaller than those given by Roussel et al. (2001b) for the galaxies in common. The reason for that is that the errors quoted here do not include those due to incomplete short-term transient correction. These were estimated on a case by case basis by Roussel et al. (2001b) using a method that is impractical to implement on a larger sample such as this one. This comparison clearly shows the dominant impact of short-term transient on ISOCAM photometry.

8 As explained in the previous section, $r_{\lambda}$ is on average $\sim 20 \%$ larger than the mid-IR major axis radius of the galaxy. 
We performed an internal check on the accuracy of the photometry by comparing total flux densities extracted from images independently reduced with different parameters. This test has been done on both LW2 and LW3 images of 20 galaxies. The consistency on the flux densities extracted on images reduced using different parameters for the long-term transient and flat-field corrections (see Miville-Deschênes et al. 2000 for details) is generally within $\sim 15 \%$. Only a few objects where the gradient in the sky background has not been succesfully removed by the task IMSURFIT (see previous section) have larger inconsistencies.

These tests, however, do not give an estimate of the systematic error since they are based on the analysis of nonindependent data. The galaxy VCC 1686 has two independent datasets since it has been observed twice during the ISO Virgo observations: once with a dedicated raster, and once as a serendipitous observation in the raster field of VCC 1690. The results of the different observations are given in Table 3.

Even if the discrepancy between the two observations are high, the two sets of data are consistent within the errors. We do not know whether this case is representative of weak sources (we however detect sources $\sim 10$ times weaker than VCC 1686), or holds also for brighter sources, with higher signal to noise. For general purposes we can assume that the photometric error on the absolute flux densities of these ISOCAM images is $\sim 30 \%$. We warn however of conceiving the ISOCAM errors as a constant fraction of the object's flux. This is generally wrong: for most objects observed with ISOCAM, the major source of noise is the zodiacal background, which is often much brighter than the object itself. Errors quite routinely reach a few $\%$ to less than $1 \%$ of the background level (as is clearly the case for the ISO-Virgo sample observations). Depending on the source brightness however, this can lead to much larger errors, when expressed as a function of the source flux.

\subsection{Resolved galaxies}

Thirty-four galaxies have been fully resolved by ISOCAM at $6.75 \mu \mathrm{m}$. Their images (obtained after a rebinning to $1 \mathrm{arc}-$ sec/pixel using a linear interpolation) are shown in Fig. 1 as grey levels. All images of late-type galaxies are characterized by a strong nuclear emission, a diffuse and extended emission, and by the emission of the brightest spiral patterns (see for instance VCC 1555 or VCC 1727). Early-type galaxies such as VCC 1978 show only a nuclear and a diffuse emission.

Radial profiles and colours of the near-IR images (Fig. 1) have been obtained using the same procedure adopted in Gavazzi et al. (2000). For each rebinned frame the sky background was determined in regions of "empty" sky, and it was subtracted from the frame. Sky-subtracted frames were inspected individually and the light of unwanted superposed or nearby stars and galaxies was masked.

The 2-dimensional light distribution of each galaxy was fitted with elliptical isophotes, using a modified version of the STSDAS ISOPHOTE package. Starting from a set of initial parameters given manually, the fit maintains as free parameters the ellipse center, ellipticity and position angle. The ellipse semi-major axis is incremented by a fixed fraction of its value at each step of the fitting procedure. The routine halts when the surface brightness found in a given corona equals the sky rms. The fit fails to converge for some galaxies with very irregular light distributions. In these cases we keep fixed one or more of the initial parameters. The resulting radial light profiles are fitted with models of the galaxy light distribution: a de Vaucouleurs $r^{1 / 4}$ law (de Vaucouleurs 1948) or an exponential disc law, or a combination of the two. Total flux densities are then obtained by adding to the flux density measured within the outermost significant isophote the value extrapolated to infinity along either the $r^{1 / 4}$ ( $\mathrm{dV}$ galaxies), or the exponential law that fitted the outer parts of the galaxy (pure discs and $\mathrm{B}+\mathrm{D}$ galaxies $)^{9}$. This routine gives also the concentration index $\left(C_{31}\right)$, defined in de Vaucouleurs (1977) as the model-independent ratio between the radii that enclose $75 \%$ and $25 \%$ of the total flux. Values of $C_{31} \leq 3$ are for pure exponential discs, $>3$ for galaxies with prominent bulges and/or nuclei.

Results of the fitting procedures are given in Table 4, arranged as follows:

- Column 1: VCC galaxy name.

- Column 2: $C_{31}(6.75)$ concentration index at $6.75 \mu \mathrm{m}$.

- Column 3: $6.75 \mu \mathrm{m}$ isophotal major axis radius $r_{5}(6.75)$ at $5 \mu \mathrm{Jy} \operatorname{arcsec}^{-2}$, in arcsec.

- Column 4: $6.75 \mu \mathrm{m}$ effective radius $R_{\mathrm{e}}(6.75)$, the radius containing half of the total light, in arcsec.

- Column 5: $6.75 \mu_{\mathrm{e}}(6.75)$ effective surface brightness, the mean surface brightness within $R_{\mathrm{e}}(6.75)$, in $\mu \mathrm{Jy} \operatorname{arcsec}^{-2}$.

- Columns 6, 7, 8 and 9: $15 \mu \mathrm{m} C_{31}(15)$ concentration index, isophotal major axis radius $r_{5}(15)$, effective radius $R_{\mathrm{e}}(15)$ and surface brightness $\mu_{\mathrm{e}}(15)$.

Light profiles are reliable only outside the diffraction limit of each filter, the colour profile outside 8 arcseconds, which represents the diffraction limit at $15 \mu \mathrm{m}$, as indicated by the vertical dashed line in the $F(6.75) / F(15)$ colour profile given in Fig. 1. The error in the light profile is dominated by the presence of large scale gradients in the sky background, left over by the flat-fielding procedure. These errors affects mostly the large scale, low surface brightness extended regions.

Late-type galaxies have a variety of mid-IR light profiles, from pure exponential discs such as VCC 66 to bulge plus disc profile (VCC 1110), or irregular profiles such as VCC 92.

Mid-IR and optical isophotal radii are strongly related one another (see Fig. 3).

The best fit gives the following relationships:

$$
r_{5}(6.75)=0.375 \times(a / 2)+8.321 \quad R^{2}=0.52
$$

and

$r_{5}(15)=0.408 \times(a / 2)+2.428 \quad R^{2}=0.46$.

These relationships are used to estimate the mid-IR extension of undetected sources (see Sect. 4).

\footnotetext{
${ }^{9}$ For homogeneity, the flux densities of the 34 fully resolved galaxies given in Table 2 are not the extrapolated values obtained by this fitting procedure but are those obtained with the QPHOT task described in Sect. 4.
} 
Table 3. Flux density estimates for the two independent observations of VCC 1686.

\begin{tabular}{|c|c|c|c|c|c|c|c|c|}
\hline $\begin{array}{l}\text { Frame } \\
\text { (1) }\end{array}$ & $\begin{array}{c}F(6.75) \\
\text { mJy } \\
(2)\end{array}$ & $\begin{array}{c}\sigma(s)_{6.75} \\
\mu \mathrm{Jy} \operatorname{arcsec}^{-2} \\
(3)\end{array}$ & $\begin{array}{c}\sigma(p)_{6.75} \\
\mu \mathrm{Jy} \operatorname{arcsec}^{-2} \\
\quad(4)\end{array}$ & $\begin{array}{c}B g(6.75) \\
\mu \mathrm{Jy} \operatorname{arcsec}^{-2} \\
(5)\end{array}$ & $\begin{array}{c}F(15) \\
\mathrm{mJy} \\
(6)\end{array}$ & $\begin{array}{c}\sigma(s)_{15} \\
\mu \mathrm{Jy} \operatorname{arcsec}^{-2} \\
(7)\end{array}$ & $\begin{array}{c}\sigma(p)_{15} \\
\mu \mathrm{Jy} \operatorname{arcsec}^{-2} \\
(8)\end{array}$ & $\begin{array}{c}B g(15) \\
\mu \mathrm{Jy} \operatorname{arcsec}^{-2} \\
(9)\end{array}$ \\
\hline 1686 & $20.39 \pm 1.73$ & 0.2 & 0.5 & 144.0 & $10.63 \pm 3.93$ & 0.6 & 1.0 & 1085.0 \\
\hline 1690 & $27.60 \pm 4.29$ & 0.5 & 0.7 & 143.9 & $20.94 \pm 10.7$ & 1.3 & 1.8 & 872.0 \\
\hline
\end{tabular}

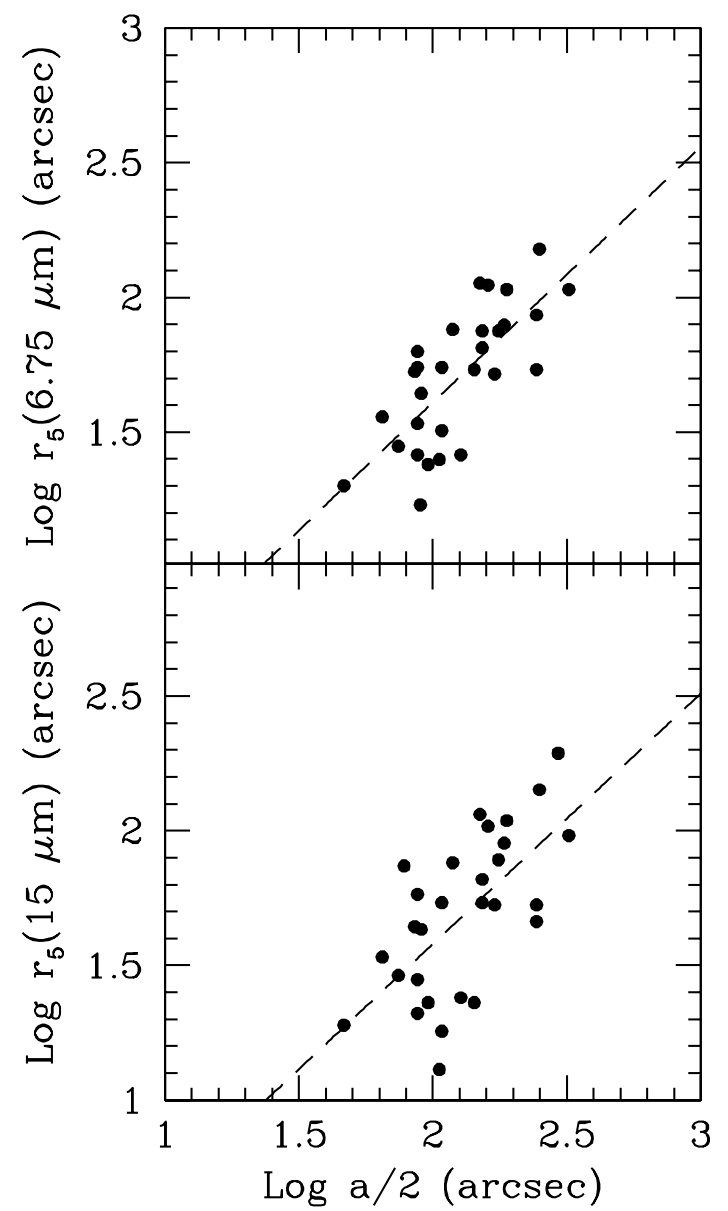

Fig. 3. The relationship between mid-IR and optical isophotal radii. The dashed line is the best fit given in Eqs. (5) and (6).

\section{Comparison with the literature}

The mid-IR data of the ISO Virgo sample and of the A1367 and Coma clusters sample were previously processed by Boselli et al. (1997a, 1998) and Contursi et al. (2001) using CIA v1.0 and v2.0. Compared to these versions, the current processing incorporates, in increasing order of impact of the quality and accuracy of the photometry: (1) a slight variation of the calibration factors $(\sim 1-2 \%)(2)$ an accurate modeling of the time dependency of the dark current, (3) time variable flat-fielding, (4) removal of the long-term transient, and (5) a better modeling of the short-term transients that in particular takes into account the fact that downward transients are different from upward ones.
Roussel et al. (2001b) published 6.75 and $15 \mu \mathrm{m}$ flux densities for 23 of the brightest Virgo galaxies, obtained reducing the data using CIA v3.0, i.e. with time-dependent dark correction, physical modeling of the short-term transient, but with a rather simplistic implementation of Miville-Deschênes et al. (2000) methods. Comparison between the mid-IR flux densities obtained in this work with those available in the literature is shown in Figs. 4a,b.

The different reduction processes produce consistent sets of data. The new data reduction process used in this work significantly reduces the noise in the ISOCAM images with respect to the early pipeline, allowing a higher detection rate at low luminosity and low surface brightness Virgo cluster galaxies. In the present work we have 13 and 11 more detections than in Boselli et al. (1998) at $6.75 \mu \mathrm{m}$ and $15 \mu \mathrm{m}$ respectively.

The ratio of the mid-IR flux densities obtained in this work to those given in the literature is however constant only for the brightest two thirds of the sample $(F(6.75 \mu \mathrm{m}) \geq 6 \mathrm{mJy}$, $F(15 \mu \mathrm{m}) \geq 10 \mathrm{mJy})$. For the weaker sources Boselli et al. (1998) and Contursi et al. (2001) overestimated their fluxes.

The impact of the new photometry on the scientific results presented in Boselli et al. (1997a, 1998) are presented in Boselli et al. (2003) and will be further discussed in future communications.

\section{Analysis}

The ratio of the observed flux densities at 6.75 and $15 \mu \mathrm{m}$ can be used to study the origin of the emission at these wavelengths. Figure 5 shows the relationships between the 6.75 to $15 \mu \mathrm{m}$ flux density ratio and various structural parameters of the sample galaxies, i.e. the $H$ band luminosity (Fig. 5a), which is a direct tracer of the total dynamical mass (Gavazzi et al. 1996), the morphological type (Fig. 5b), the $K$ concentration index (Fig. 5c), which is indicative of the spatial distribution of the old stellar population within galaxies, and the $C_{31}(6.75)$ index (Fig. 5d), available only for the resolved galaxies.

Figure 5a shows that the observed mid-IR colour index shows a weak trend with the total mass: massive galaxies have $F(6.75 \mu \mathrm{m}) / F(15 \mu \mathrm{m})$ higher than low mass objects. All early-type galaxies have $F(6.75 \mu \mathrm{m}) / F(15 \mu \mathrm{m})>1$, while in late-types the $F(6.75 \mu \mathrm{m}) / F(15 \mu \mathrm{m})$ ratio ranges between $\sim 0.3$ and 5. No relation is observed between the mid-IR colour index and the underlying stellar distribution. Five out of six galaxies with a mid-IR colour $F(6.75 \mu \mathrm{m}) / F(15 \mu \mathrm{m})>3$ have a peaked emission at $6.75 \mu \mathrm{m}\left(C_{31}(6.75)>5\right)$, while $\sim 95 \%$ of the objects with an exponential light profile at $6.75 \mu \mathrm{m}$ have $F(6.75 \mu \mathrm{m}) / F(15 \mu \mathrm{m}) \leq 2$. 

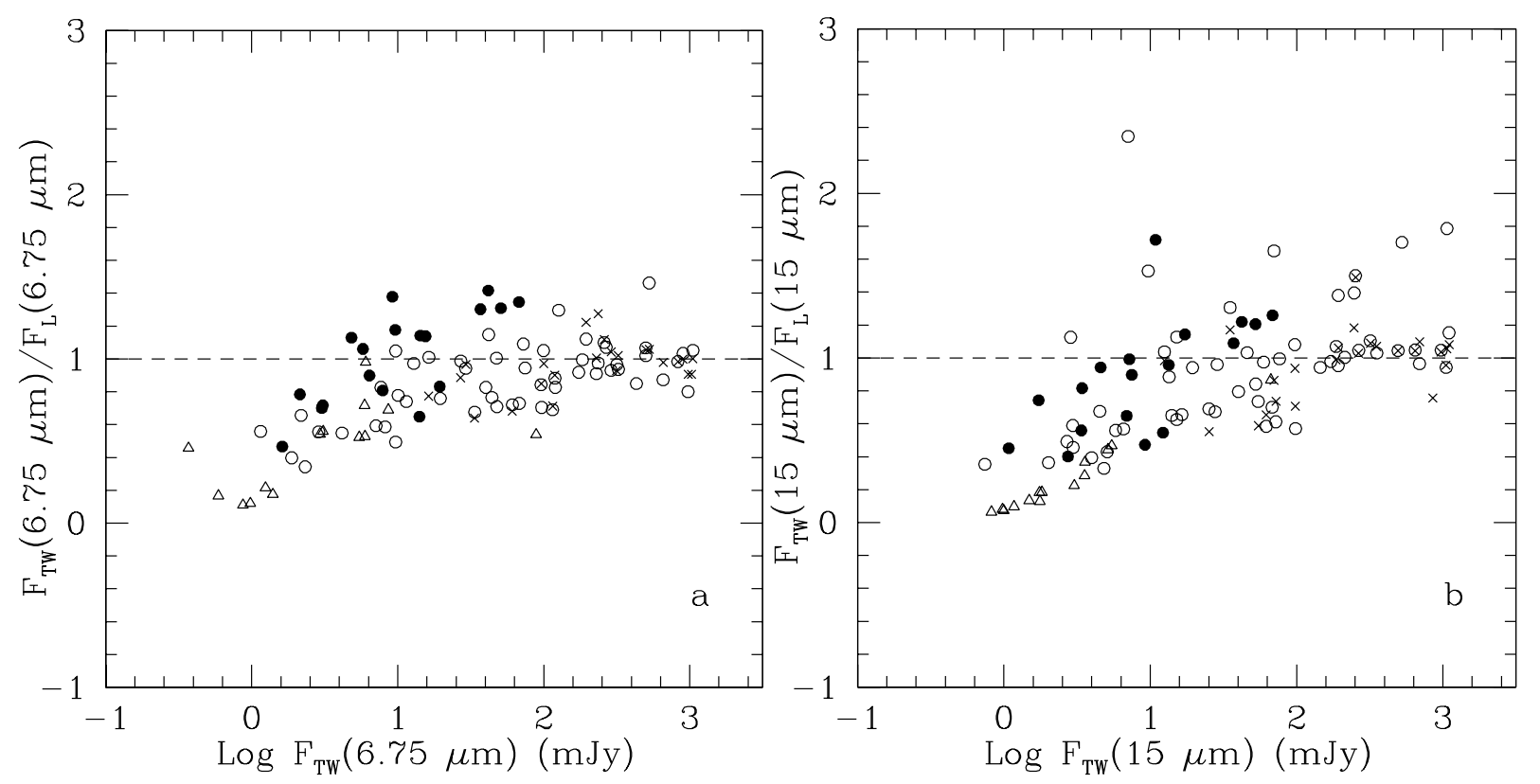

Fig. 4. The ratio of the flux densities obtained in this work with those available in the literature versus our flux densities at a) $6.75 \mu \mathrm{m}$ and b) $15 \mu \mathrm{m}$. The dashed line indicates a linear relation between the two sets of data. Crosses are for Roussel et al. (2001b), filled dots for Contursi et al. (2001), while open dots and open triangles are for detected and undetected (upper limits) galaxies in Boselli et al. (1998), respectively.
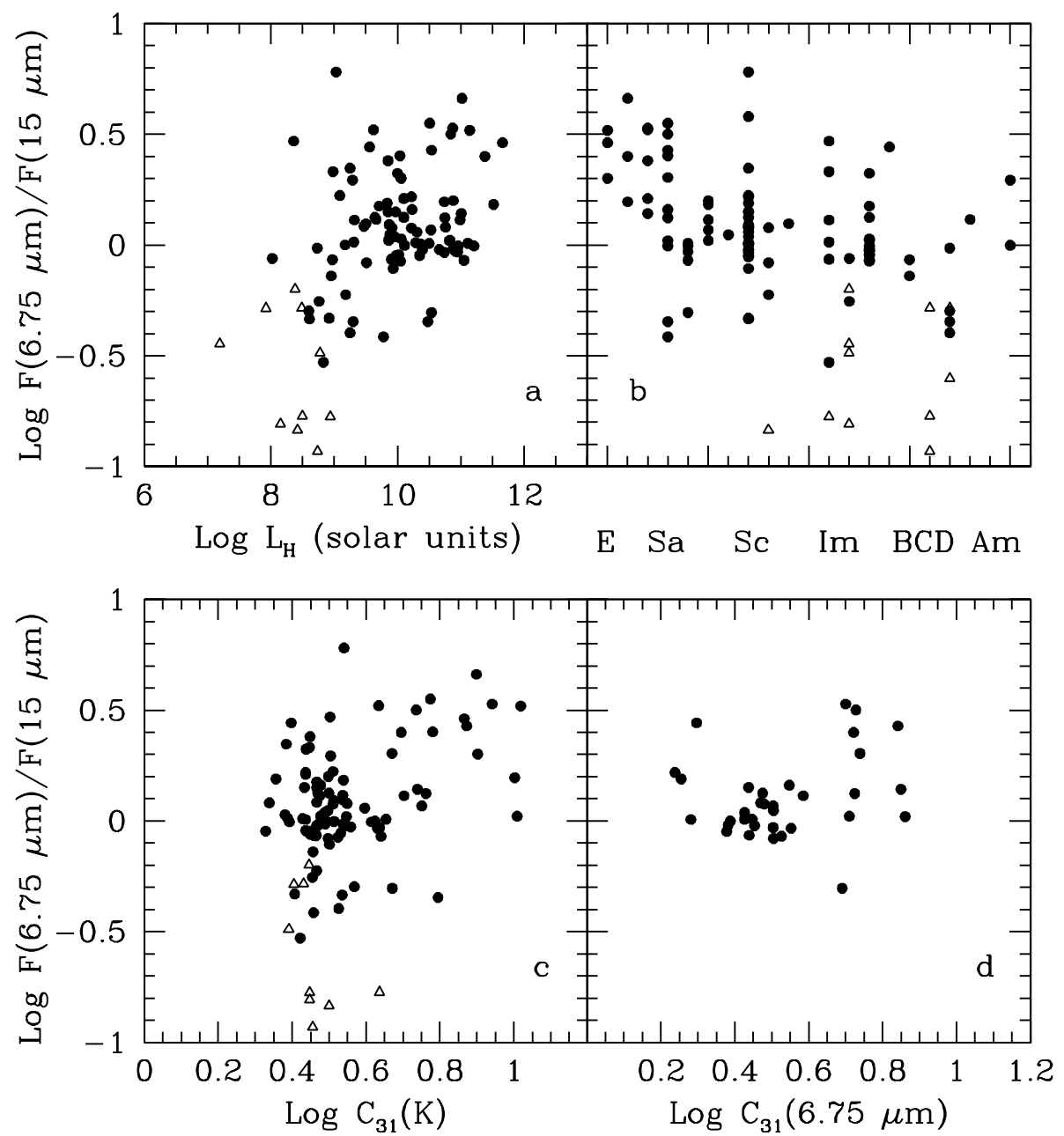

Fig. 5. The relationship between the mid-IR colour index $F(6.75 \mu \mathrm{m}) / F(15 \mu \mathrm{m})$ and a) the total mass (as traced by the $H$ band luminosity), b) the morphological type, $\mathbf{c}$ ) the $K$ band $C_{31}(K)$ concentration index and d) the mid-IR $C_{31}(6.75)$ concentration index. Filled dots are for galaxies detected at 6.75 and $15 \mu \mathrm{m}$, open triangles for galaxies undetected at $15 \mu \mathrm{m}$. 


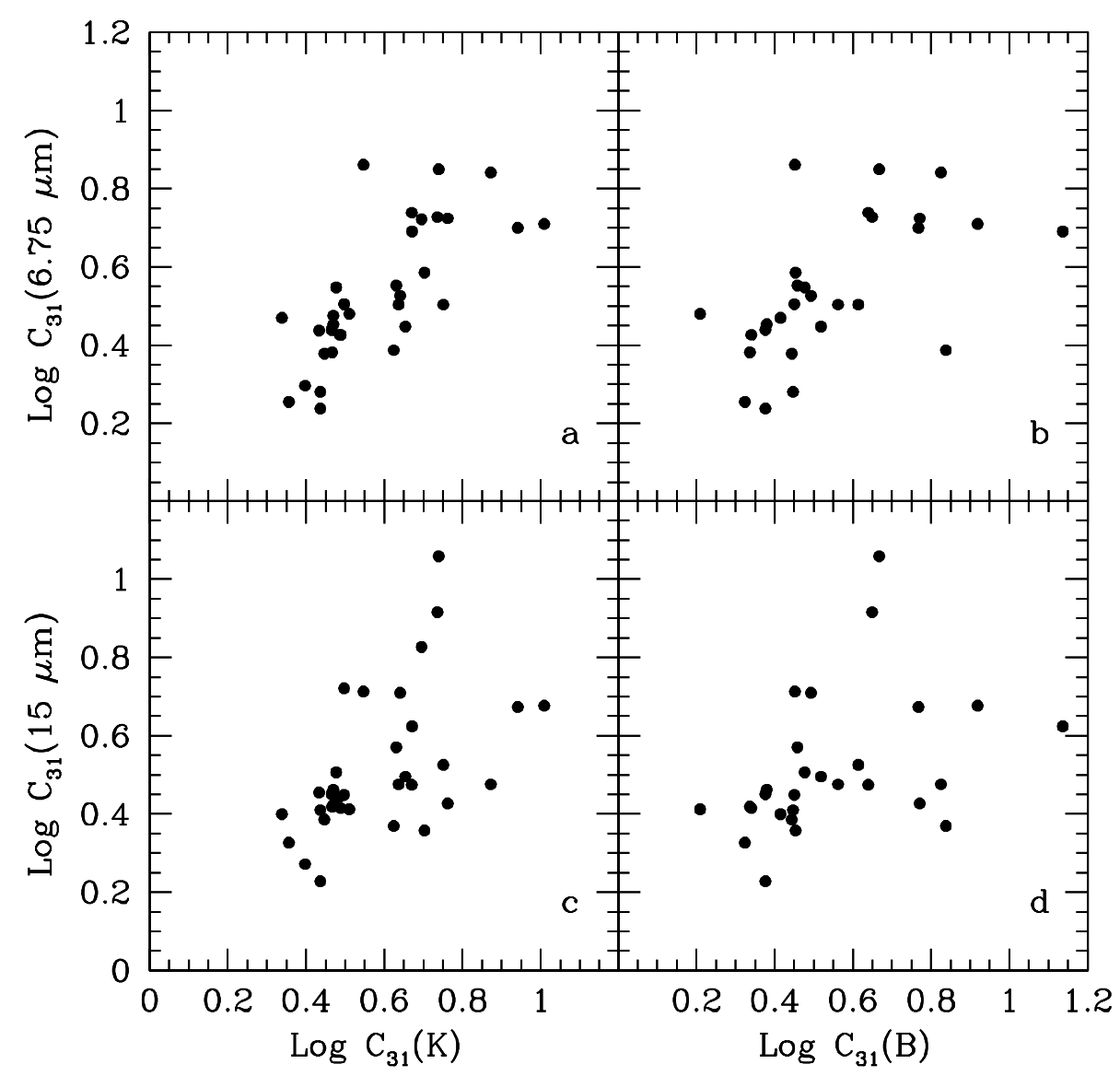

Fig. 6. The relationship between the mid-IR and the near-IR and visible concentration indices.

The relationships between the mid-IR and the near-IR and visible concentration indices (this last being weak), shown in Fig. 6, indicate that bulge dominated galaxies $\left(C_{31}(K) \geq 3\right)$ have peaked mid-IR distributions.

High values of $F(6.75 \mu \mathrm{m}) / F(15 \mu \mathrm{m})$ are expected when the contribution of the black-body tail of the cold stellar population to the total mid-IR emission becomes important: our observational evidences indicate that the contribution of the old stellar population to the mid-IR emission is important in earlytype and/or massive galaxies, with prominent bulges, with a centrally peaked mid-IR light distribution. Dust emission is dominant in low-mass objects characterized by an exponential distribution of the visible, near- and mid-IR light. This picture is coherent with our previous results given in Boselli et al. (1998), and confirmed by our recent analysis of the spectral energy distribution (SED) presented in Boselli et al. (2003).

No strong relationships are observed between the ratio of the mid-IR effective radii and surface brightnesses and other structural parameters, such as the $H$ band luminosity, the morphological type and the $C_{31}(K)$ concentration index. A weak trend is however observed between the ratio of the mid-IR $6.75 \mu \mathrm{m}$ to visible isophotal radii and the morphological type (if limited to spirals) and $C_{31}(K)$ concentration index (Fig. 7), indicating that the mid-IR disc is more extended in Sc galaxies characterized by exponentially declining light distributions (both near- and mid-IR) than in bulge-dominated Sa.
Colour profiles are generally flat, with an average value of $F(6.75 \mu \mathrm{m}) / F(15 \mu \mathrm{m})$ flux density ratio of $\sim 1$. Only a few objects present peculiar colour profiles: some galaxies have 6.75 to $15 \mu \mathrm{m}$ flux densities ratios lower than one in the central region (VCC 836, and to a lower extent VCC 460, VCC 1003, VCC 1690, VCC 1727 and VCC 1813). All but one (VCC 1813) are active galaxies (Syfert, LINER). Laurent et al. (2000) have shown that in active galaxies such as AGN the $F(6.75 \mu \mathrm{m}) / F(15 \mu \mathrm{m})$ ratio is expected to be lower than one. On the contrary, the Sa VCC 1158 and VCC 2070 have 6.75 to $15 \mu \mathrm{m}$ flux densities ratios increasing towards the centre probably due to an higher contribution at $6.75 \mu \mathrm{m}$ than at $15 \mu \mathrm{m}$ of the old stellar component. The two resolved S0a galaxies VCC 1253 and VCC 1978 have flat colours, with a 6.75 to $15 \mu \mathrm{m}$ flux density ratio $\geq 2$. The emission is diffuse without any evident morphological structure. 6.75 to $15 \mu \mathrm{m}$ flux density ratio $\geq 2$ in early-type galaxies are easely obtained if their mid-IR emission is dominated by the cold stellar population. Morphological considerations are thus in total agreement with those obtained by the analysis of the SED (Boselli et al. 2003).

\section{Conclusion}

We present new ISOCAM imaging data taken at 6.75 and $15 \mu \mathrm{m}$ for a complete, optically-selected, volume-limited sample of 99 late-type ( $\geq$ S0a) galaxies in the Virgo cluster. This sample is composed of 71 objects in the cluster periphery, with 

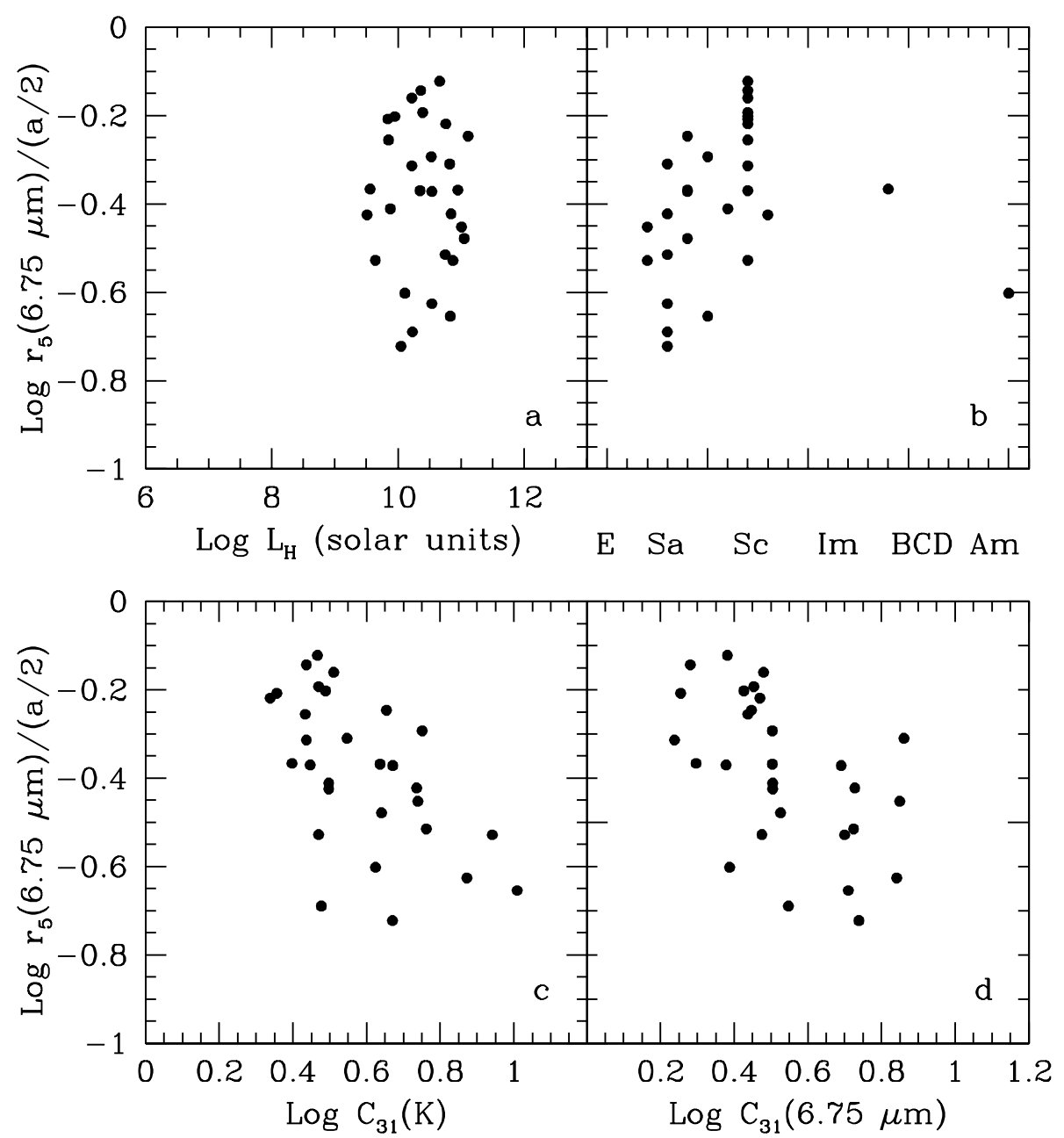

Fig. 7. The relationship between the mid-IR $6.75 \mu \mathrm{m}$ to visible isophotal radii and a) the total mass (as traced by the $H$ band luminosity), b) the morphological type, c) the $K$ band $C_{31}(K)$ concentration index and d) the mid-IR $C_{31}(6.75)$ concentration index.

a projected angular distance $\theta \geq 4^{\circ}$ from $\mathrm{M} 87$, and 28 in the cluster core $\left(\theta \leq 2^{\circ}\right)$. ISOCAM data for another 24 galaxies in the Virgo cluster serendipitously observed in the same frame of other objects (18 galaxies), or found in the ISOCAM data archive (6 galaxies), are also presented. To these we add 22 galaxies in the Coma/A1367 supercluster, out of which 13 are Coma members and 6 are in A1367.

All the data here presented are reduced and analysed in a homogeneous way. Out of the 145 observed galaxies, 34 objects, all in the Virgo cluster, were fully resolved by ISOCAM: for these objects we present images, light profiles and colour profiles, as well as effective and isophotal radii, surface brightnesses and light concentration indices.

Our analysis shows that the mid-IR colours of the target galaxies are weakely correlated with the total mass of galaxies, as traced by the $H$ band luminosity, with high values of $F(6.75 \mu \mathrm{m}) / F(15 \mu \mathrm{m})(\geq 1)$ found generally in massive objects $\left(L_{H} \geq 10^{10} L_{H \odot}\right)$, and $F(6.75 \mu \mathrm{m}) / F(15 \mu \mathrm{m}) \leq 1$ in low-mass $\left(L_{H} \leq 10^{10} L_{H \odot}\right)$ dwarf galaxies. All early-type galaxies (type $\leq$ S0a) have $F(6.75 \mu \mathrm{m}) / F(15 \mu \mathrm{m}) \geq 1$, as expected if the midIR emission is dominated by the photosphere of the cold stellar population.
The mid-IR, near-IR and visible light concentration indices are tightly correlated in bright, resolved galaxies, indicating that the spatial distribution of the mid-IR emitting sources is, to the first order, similar to that of the stars.

The analysis on the origin of the mid-IR emission of latetype galaxies is postponed to a forthcoming communication.

Acknowledgements. A.B. thanks the CEA team for the support during his staying in Saclay. We thank G. Sanvito and J. Iglesias for assistence in the preparation of the figures. We wish to thank R. Tuffs, the referee, for his useful comments and suggestions which helped improving the quality of the manuscript. This work is partly financed by the French Programe National Physique et Chimie du Milieu Interstellaire.

\section{References}

Bendo, G. J., Joseph, R. D., Wells, M., et al. 2002, AJ, 123, 3067

Binggeli, B., Sandage, A., \& Tammann, G. A. 1985, AJ, 90, 1681 (VCC)

Binggeli, B., Popescu, C. C., \& Tammann, G. A. 1993, A\&AS, 98, 275

Boselli, A., Lequeux, J., Contursi, A., et al. 1997a, A\&A, 324, L13

Boselli, A., Tuffs, R. J., Gavazzi, G., Hippelein, H., \& Pierini, D. 1997b, A\&A, 121, 507 
Boselli, A., Lequeux, J., \& Sauvage, M. 1998, et al. A\&A, 335, 53

Boselli, A., Gavazzi, G., Lequeux, J., \& Pierini, D. 2002, A\&A, 385, 454

Boselli, A., Gavazzi, G., \& Sanvito, G. 2003, A\&A, 402, 37

Contursi, A., Boselli, A., Gavazzi, G., et al. 2001, A\&A, 365, 11

Coulais, A., \& Abergel, A. 2000, A\&AS, 141, 533

Cox, P., \& Mezger, P. 1989, A\&ARv, 1, 49

Dale, D. A., Silbermann, N. A., Helou, G., et al. 2000, AJ, 120, 583

Désert, F.-X., Boulanger, F., \& Puget, J.-L. 1990, A\&A, 237, 215

de Vaucouleurs, G. 1948, Ann. Astrophys., 11, 247

de Vaucouleurs, G. 1977, in Evolution of Galaxies and Stellar Populations, ed. R. Larson, \& B. Tinsley (New Haven: Yale University Observatory), 43

Draine, B. T., \& Li, A. 2001, ApJ, 551, 807

Dwek, E., Arendt, R., Fixsen, D., et al. 1997, ApJ, 475, 565

Elbaz, D., Cesarsky, C. J., Chanial, P., et al. 2002, A\&A, 384, 848

Gavazzi, G., \& Boselli, A. 1996, Astro. Lett. Commun., 35, 1

Gavazzi, G., Pierini, D., \& Boselli, A. 1996, A\&A, 312, 397

Gavazzi, G., Boselli, A., Scodeggio, M., Pierini, D., \& Belsole, E. 1999a, MNRAS, 304, 595

Gavazzi, G., Carrasco, L., \& Galli, R. 1999b, A\&AS, 136, 227

Gavazzi, G., Franzetti, P., Scodeggio, M., Boselli, A., \& Pierini, D. 2000, A\&A, 361, 863

Gavazzi, G., Marcelin, M., Boselli, A., et al. 2001, A\&A, 377, 745

Laurent, O., Mirabel, L., Charmandaris, V., et al. 2000, A\&A, 359, 887

Lagache, G., Abergel, A., Boulanger, F., Désert, F. X., \& Puget, J.-L. 1999, A\&A, 344, 322

Leech, K., Völk, H., Heinrichsen, I., et al. 1999, MNRAS, 310, 317
Li, A., \& Draine, B. T. 2001, ApJ, 554, 778

Miville-Deschenes, M. A., Boulanger, F., Abergel, A., \& Bernard, J. P. 2000, A\&AS, 146, 519

Nilson, P. 1973, Uppsala General Catalogue of Galaxies, Uppsala Obser. Annu., Vol. 6 (UGC)

Pierini, D., Leech, K. J., Tuffs, R. J., \& Völk, H. J. 1999, MNRAS, 303, L29

Pierini, D., Lequeux, J., Boselli, A., Leech, K. J., \& Völk, H. J. 2001, A\&A, 373, 827

Popescu, C. C., Misiriotis, A., Kylafis, N. D., Tuffs, R. J., \& Fishera, J. 2000, A\&A, 362, 138

Popescu, C. C., Tuffs, R. J., Völk, H. J., Pierini, D., \& Madore, B. F. 2002, ApJ, 567, 221

Popescu, C. C., \& Tuffs, R. J. 2002, MNRAS, 335, L41

Ristorcelli, I., Serra, G., Lamarre, J. M., et al. 1999, in Solid Interstellar Matter: The ISO Revolution, ed. L. d'Hendecourt, C. Joblin, \& A. Jones (EDP Sciences and Springer-Verlag), 49

Roussel, H., Sauvage, M., Vigroux, L., \& Bosma, A. 2001a, A\&A, 372, 427

Roussel, H., Vigroux, L., Bosma, A., et al. 2001b, A\&A, 369, 473

Sandage, A., Binggeli, B., \& Tammann, G. A. 1985, AJ, 90, 1759

Siebenmorgen, R., Blommaert, J., Sauvage, M., \& Starck, J. L. 2000, The ISO handbook. III. CAM - the ISO camera, ESA, SAI-99057/Dc, Version 1.1

Starck, J. L., Abergel, A., Aussel, A., et al. 1999, A\&AS, 134, 135

Tuffs, R. J., Popescu, C. C., Pierini, D., et al. 2002, ApJS, 139, 37

Zwicky, F., Herzog, E., Karpowicz, M., Kowal, C., \& Wild, P. 19611968, Catalogue of Galaxies and of Cluster of Galaxies (Pasadena, California Institute of Technology: CGCG) 
A. Boselli et al.: Mid-IR emission of galaxies in the Virgo cluster. III., Online Material p 1

\section{Online Material}


Table 1. The target galaxies.

\begin{tabular}{|c|c|c|c|c|c|c|c|c|c|c|c|c|c|c|}
\hline \multicolumn{15}{|c|}{ The ISO Virgo sample } \\
\hline $\mathrm{VCC}$ & UGC & NGC/IC & RA(2000) & dec & Type & $m_{p g}$ & $a$ & $b$ & Dist & Memb. & $\theta$ & $C_{31}(K)$ & $C_{31}(B)$ & notes \\
\hline (1) & & (3) & h $\mathrm{m} \mathrm{s}$ & $\begin{aligned} o & , \quad 1 \prime \\
& (5)\end{aligned}$ & (6) & & (8) & $\begin{array}{l}\text { ' } \\
\text { (9) }\end{array}$ & $\begin{array}{r}\text { Mpc } \\
(10)\end{array}$ & & $\begin{array}{l}\text { deg } \\
(12)\end{array}$ & (13) & (14) & (15) \\
\hline 1 & - & - & 120820.02 & 134100.2 & BCD? & 14.78 & 0.80 & 0.18 & 32 & $\mathrm{M}$ & 5.63 & 3.36 & 2.68 & \\
\hline 4 & - & - & 120830.75 & 150548.2 & $\mathrm{Im}$ & 17.50 & 0.50 & 0.43 & 32 & M & 6.06 & - & - & \\
\hline 17 & 7150 & 3023 & 121001.86 & 142142.4 & Im & 15.20 & 0.91 & 0.45 & 32 & M & 5.43 & 2.85 & 2.37 & \\
\hline 24 & - & - & 121035.65 & 114538.5 & $\mathrm{BCD}$ & 14.95 & 1.00 & 0.37 & 32 & M & 4.99 & 6.23 & 2.78 & \\
\hline 26 & - & - & 121040.20 & 143848.5 & $\mathrm{Im}$ & 17.50 & 0.43 & 0.27 & 32 & $\mathrm{M}$ & 5.39 & 2.35 & - & \\
\hline 66 & 7215 & 4178 & 121246.27 & 105156.0 & $\mathrm{SBc}(\mathrm{s})$ & 11.89 & 5.35 & 1.87 & 17 & $\mathrm{~N}$ & 4.68 & 3.24 & 1.62 & $*$ \\
\hline 81 & 7223 & 4186 & 121326.18 & 144620.1 & $\mathrm{~d}: \mathrm{Sc}$ & 15.60 & 0.95 & 0.81 & 17 & $\mathrm{~N}$ & 4.85 & 3.22 & 2.64 & \\
\hline 87 & - & - & 121340.91 & 152713.2 & $\mathrm{Sm}$ & 15.00 & 1.45 & 0.72 & 17 & $\mathrm{~N}$ & 5.17 & 3.18 & - & \\
\hline 92 & 7231 & 4192 & 121348.24 & 145401.2 & $\mathrm{Sb}$ : & 10.92 & 9.78 & 2.60 & 17 & $\mathrm{~N}$ & 4.84 & 5.04 & 2.84 & $*$ \\
\hline 130 & - & - & 121504.22 & 94513.5 & BCD & 16.50 & 0.63 & 0.25 & 17 & $\mathrm{~N}$ & 4.68 & 2.71 & - & \\
\hline 152 & 7268 & 4207 & 121530.31 & 93508.6 & Scd(on edge) & 13.48 & 1.96 & 0.89 & 17 & $\mathrm{~N}$ & 4.69 & 3.54 & 2.21 & \\
\hline 159 & - & - & 121541.50 & 81707.7 & $\mathrm{Im}$ & 15.08 & 1.04 & 0.52 & 32 & W & 5.54 & 2.73 & - & \\
\hline 169 & - & - & 121556.39 & 93855.7 & $\mathrm{Im}$ & 16.50 & 0.85 & 0.43 & 17 & $\mathrm{~N}$ & 4.57 & - & - & \\
\hline 171 & - & - & 121558.88 & 82225.8 & Im & 17.40 & 0.57 & 0.36 & 32 & $\mathrm{~W}$ & 5.43 & - & - & \\
\hline 207 & - & - & 121648.07 & 80302.0 & $\mathrm{BCD}$ & 17.20 & 0.36 & 0.13 & 32 & W & 5.55 & 2.63 & - & \\
\hline 318 & 7352 & 776 & 121903.40 & 85122.7 & SBcd & 14.01 & 1.71 & 1.00 & 32 & W & 4.57 & 2.93 & - & \\
\hline 425 & - & - & 122035.90 & 81209.3 & Im: & 17.30 & 0.43 & 0.38 & 23 & B & 4.89 & - & - & \\
\hline 459 & - & - & 122111.46 & 173818.5 & $\mathrm{BCD}$ & 14.95 & 0.84 & 0.36 & 17 & A & 5.74 & 3.09 & - & \\
\hline 460 & 7405 & 4293 & 122112.68 & 182256.5 & Sa pec & 11.20 & 5.10 & 2.92 & 17 & A & 6.42 & 3.52 & 2.83 & $*$ \\
\hline 655 & 7468 & 4344 & 122337.45 & 173228.5 & S pec,N:/BCD & 13.21 & 1.55 & 1.55 & 17 & A & 5.44 & 2.50 & - & \\
\hline 664 & 7470 & 3258 & 122344.36 & 122842.5 & $\mathrm{Sc}$ & 13.50 & 2.60 & 1.87 & 17 & A & 1.73 & 2.55 & - & \\
\hline 666 & - & - & 122346.13 & 164728.5 & Im: & 16.80 & 1.00 & 0.57 & 17 & A & 4.72 & 2.80 & - & \\
\hline 692 & 7476 & 4351 & 122401.37 & 121216.6 & $\mathrm{Sc}(\mathrm{s})$ & 12.93 & 2.92 & 1.87 & 17 & A & 1.67 & 2.95 & - & \\
\hline 809 & 7510 & 3311 & 122533.17 & 121536.3 & Sc (on edge) & 14.55 & 1.45 & 0.36 & 17 & A & 1.30 & 3.24 & - & \\
\hline 836 & 7520 & 4388 & 122546.60 & 123940.4 & Sab & 11.83 & 5.10 & 1.24 & 17 & A & 1.26 & 4.69 & 13.70 & $*$ \\
\hline 848 & - & - & 122552.78 & 54829.5 & Im pec/BCD & 14.72 & 1.16 & 0.98 & 23 & B & 6.70 & 2.86 & - & \\
\hline 857 & 7523 & 4394 & 122555.64 & 181249.5 & $\mathrm{SBb}(\mathrm{sr})$ & 11.76 & 3.60 & 3.60 & 17 & A & 5.94 & 5.64 & 4.11 & * \\
\hline 873 & 7528 & 4402 & 122607.32 & 130643.6 & Sc (on edge) & 12.56 & 3.95 & 1.16 & 17 & A & 1.36 & 2.95 & 2.40 & \\
\hline 890 & - & - & 122620.85 & 64005.7 & BCD & 16.00 & 0.21 & 0.21 & 23 & B & 5.83 & 2.64 & - & \\
\hline 912 & 7538 & 4413 & 122632.16 & 123639.8 & $\mathrm{SBbc}(\mathrm{rs})$ & 12.97 & 2.92 & 1.75 & 17 & A & 1.07 & 3.14 & - & \\
\hline 971 & 7556 & 4423 & 122708.93 & 55248.1 & Sd (on edge) & 14.28 & 3.06 & 0.43 & 23 & B & 6.57 & 3.44 & - & \\
\hline 984 & 7562 & 4425 & 122713.30 & 124405.1 & $\mathrm{SBa}$ & 12.82 & 2.99 & 1.00 & 17 & A & 0.94 & 4.68 & 4.36 & \\
\hline 995 & 7565 & 3371 & 122721.55 & 105155.2 & Sc (on edge) & 15.32 & 1.53 & 0.11 & 17 & A & 1.75 & 3.43 & 2.69 & \\
\hline 1002 & 7566 & 4430 & 122726.37 & 61544.2 & $\mathrm{SBc}(\mathrm{r})$ & 12.48 & 3.02 & 2.69 & 23 & B & 6.19 & 2.73 & 2.38 & \\
\hline 1003 & 7568 & 4429 & 122726.31 & 110629.2 & S0/Sa pec & 11.15 & 8.12 & 3.52 & 17 & A & 1.53 & 5.48 & 4.65 & $*$ \\
\hline 1043 & 7574 & 4438 & 122745.52 & 130031.4 & $\mathrm{Sb}$ (tides) & 10.91 & 8.12 & 3.68 & 17 & A & 0.97 & 10.21 & 8.30 & * \\
\hline 1047 & 7581 & 4440 & 122753.52 & 121735.5 & $\mathrm{SBa}(\mathrm{sr})$ & 12.48 & 2.01 & 1.71 & 17 & A & 0.72 & 7.42 & - & \\
\hline 1110 & 7594 & 4450 & 122829.27 & 170506.8 & Sab pec & 10.93 & 6.15 & 4.04 & 17 & A & 4.73 & 4.33 & 3.65 & $*$ \\
\hline 1158 & 7613 & 4461 & 122903.01 & 131101.1 & $\mathrm{Sa}$ & 12.09 & 3.52 & 1.29 & 17 & A & 0.90 & 7.45 & 6.69 & \\
\hline 1189 & 7621 & 3414 & 122928.83 & 64612.3 & $\mathrm{Sc}(\mathrm{s})$ & 13.70 & 1.84 & 1.07 & 17 & S & 5.63 & 2.42 & - & \\
\hline 1196 & 7628 & 4468 & 122931.25 & 140258.3 & $\mathrm{~S} 0 / \mathrm{Sa}$ & 13.80 & 1.76 & 1.06 & 17 & A & 1.69 & 4.31 & - & \\
\hline 1217 & 7630 & 3418 & 122942.54 & 112404.4 & $\mathrm{SBm}$ & 14.59 & 1.87 & 1.29 & 17 & A & 1.03 & 2.80 & - & \\
\hline 1253 & 7638 & 4477 & 123002.37 & 133810.6 & $\mathrm{SB} 0 / \mathrm{SBa}$ & 11.31 & 3.60 & 3.60 & 17 & A & 1.26 & 8.73 & 5.86 & * \\
\hline 1257 & - & - & 123004.68 & 172401.6 & Im pec & 16.50 & 1.36 & 0.32 & 17 & A & 5.01 & 2.55 & - & \\
\hline 1326 & 7657 & 4491 & 123057.15 & 112859.1 & $\mathrm{SBa}(\mathrm{s})$ & 13.41 & 1.89 & 0.94 & 17 & A & 0.91 & 2.87 & - & \\
\hline 1368 & 7665 & 4497 & 123132.79 & 113736.4 & $\mathrm{SB} 0 / \mathrm{SBa}$ & 13.12 & 2.01 & 0.85 & 17 & A & 0.78 & 2.81 & - & \\
\hline 1379 & 7669 & 4498 & 123139.62 & 165107.5 & $\mathrm{SBc}(\mathrm{s})$ & 12.62 & 2.85 & 1.53 & 17 & A & 4.46 & 2.27 & 2.11 & \\
\hline 1410 & 7677 & 4502 & 123203.22 & 164114.7 & $\mathrm{Sm}$ & 14.57 & 1.48 & 0.78 & 17 & A & 4.31 & 2.8 & 2.91 & \\
\hline 1412 & 7680 & 4503 & 123206.13 & 111034.8 & $\mathrm{Sa}$ & 12.12 & 4.33 & 1.71 & 17 & A & 1.25 & 5.95 & 5.45 & \\
\hline 1419 & 7682 & 4506 & 123210.46 & 132509.8 & Spec(dust) & 13.64 & 2.16 & 1.29 & 17 & A & 1.08 & 3.44 & - & \\
\hline 1450 & 7695 & 3476 & 123241.91 & 140256.1 & $\mathrm{Sc}(\mathrm{s})$ & 13.29 & 2.60 & 2.01 & 17 & A & 1.72 & 2.93 & - & \\
\hline 1552 & 7729 & 4531 & 123415.77 & 130429.1 & Sa pec & 12.58 & 4.24 & 2.42 & 17 & A & 1.08 & 3 & 3.00 & \\
\hline 1554 & 7726 & 4532 & 123419.31 & 62807.1 & $\mathrm{Sm}$ & 12.30 & 2.60 & 1.00 & 17 & $\mathrm{~S}$ & 5.99 & 2.92 & 2.38 & \\
\hline 1569 & - & 3520 & 123431.68 & 133013.2 & Scd: & 15.00 & 1.07 & 0.71 & 17 & A & 1.43 & 3.16 & - & \\
\hline 1575 & 7736 & 3521 & 123439.28 & 70938.3 & SBm pec & 13.98 & 2.00 & 1.41 & 17 & $\mathrm{~S}$ & 5.32 & 2.44 & 1.99 & \\
\hline 1581 & 7739 & - & 123444.93 & 61807.4 & $\mathrm{Sm}$ & 14.55 & 1.46 & 1.16 & 17 & $\mathrm{~S}$ & 6.17 & 2.77 & - & \\
\hline 1596 & - & - & 123500.91 & 91116.5 & Im: & 17.24 & 0.35 & 0.16 & 17 & $\mathrm{~S}$ & 3.36 & - & - & \\
\hline 1673 & 7777 & 4567 & 123632.66 & 111528.6 & $\mathrm{Sc}(\mathrm{s})$ & 12.08 & 2.92 & 1.87 & 17 & A & 1.80 & 2.73 & 2.80 & * \\
\hline 1675 & - & - & 123634.65 & 80317.6 & Pec & 14.47 & 1.26 & 0.74 & 17 & $\mathrm{~S}$ & 4.56 & 2.87 & - & \\
\hline 1676 & 7776 & 4568 & 123634.16 & 111419.6 & $\mathrm{Sc}(\mathrm{s})$ & 11.70 & 5.10 & 1.75 & 17 & A & 1.82 & 4.27 & 2.87 & * \\
\hline 1678 & 7781 & 3576 & 123637.61 & 63716.6 & SBd & 13.70 & 2.16 & 1.87 & 17 & $\mathrm{~S}$ & 5.94 & 2.91 & - & $*$ \\
\hline 1686 & 7784 & 3583 & 123643.57 & 131531.7 & $\mathrm{Sm}$ & 13.95 & 2.79 & 1.71 & 17 & A & 1.68 & 2.98 & 2.41 & \\
\hline 1690 & 7786 & 4569 & 123649.78 & 130945.7 & $\mathrm{Sab}(\mathrm{s})$ & 10.25 & 10.73 & 5.35 & 17 & A & 1.65 & 4.37 & 3.11 & $*$ \\
\hline 1699 & 7790 & 3589 & 123702.24 & 65530.9 & $\mathrm{SBm}$ & 14.11 & 1.55 & 0.83 & 17 & $\mathrm{~S}$ & 5.68 & 2.64 & - & \\
\hline 1725 & - & - & 123741.51 & 83331.3 & $\mathrm{Sm} / \mathrm{BCD}$ & 14.51 & 1.55 & 0.97 & 17 & $\mathrm{~S}$ & 4.19 & 2.92 & - & \\
\hline 1726 & 7795 & - & 123745.08 & 70622.4 & Sdm & 14.54 & 1.29 & 1.00 & 17 & $\mathrm{~S}$ & 5.55 & 2.73 & - & \\
\hline 1727 & 7796 & 4579 & 123743.48 & 114904.4 & $\mathrm{Sab}(\mathrm{s})$ & 10.56 & 6.29 & 4.87 & 17 & A & 1.78 & 4.51 & 3.30 & $*$ \\
\hline 1730 & 7794 & 4580 & 123748.60 & 52206.4 & $\mathrm{Sc} / \mathrm{Sa}$ & 12.61 & 2.16 & 1.60 & 17 & $\mathrm{~S}$ & 7.23 & 2.68 & - & \\
\hline 1750 & - & - & 123815.48 & 65938.7 & $\mathrm{BCD}$ ? & 16.50 & 0.31 & 0.16 & 17 & $\mathrm{~S}$ & 5.70 & 2.54 & - & \\
\hline 1757 & 7803 & 4584 & 123817.79 & 130635.8 & $\mathrm{Sa}(\mathrm{s}) \mathrm{pec}$ & 13.60 & 1.87 & 1.00 & 17 & A & 1.96 & 3.53 & - & \\
\hline 1758 & 7802 & - & 123820.81 & 75328.8 & Sc (on edge) & 14.99 & 1.71 & 0.27 & 17 & $\mathrm{~S}$ & 4.87 & 3.47 & - & \\
\hline 1784 & - & - & 123913.81 & 153749.4 & $\mathrm{Im}$ & 15.84 & 0.79 & 0.63 & 17 & E & 3.83 & 2.80 & - & \\
\hline 1789 & - & - & 123921.34 & 45619.5 & $\operatorname{Im}$ & 15.07 & 1.10 & 0.62 & 17 & S & 7.74 & 2.46 & - & \\
\hline 1791 & 7822 & 3617 & 123924.55 & 75752.5 & $\mathrm{SBm} / \mathrm{BCD}$ & 14.67 & 1.29 & 0.64 & 17 & $\mathrm{~S}$ & 4.90 & 2.86 & - & \\
\hline 1804 & - & - & 123940.25 & 92355.7 & $\operatorname{Im} / \mathrm{BCD}$ & 15.63 & 0.75 & 0.30 & 17 & E & 3.70 & 4.33 & 3.75 & \\
\hline 1811 & 7826 & 4595 & 123951.63 & 151753.9 & $\mathrm{Sc}(\mathrm{s})$ & 12.92 & 2.16 & 1.42 & 17 & E & 3.64 & 2.71 & - & \\
\hline 1813 & 7828 & 4596 & 123955.88 & 101034.9 & SBa & 11.51 & 4.76 & 4.04 & 17 & E & 3.14 & 5.44 & 4.46 & \\
\hline 1822 & - & - & 124010.14 & 65050.1 & $\operatorname{Im}$ & 15.60 & 0.63 & 0.25 & 17 & S & 6.00 & 2.79 & 2.83 & \\
\hline
\end{tabular}


Table 1. continued.

\begin{tabular}{|c|c|c|c|c|c|c|c|c|c|c|c|c|c|c|}
\hline VCC & UGC & $\overline{\mathrm{NGC} / \mathrm{IC}}$ & $\begin{array}{c}\mathrm{RA}(2000) \\
\mathrm{h} \mathrm{m} \mathrm{s} \\
(4)\end{array}$ & $\begin{aligned} & \mathrm{dec} \\
& 0, \quad 1 \\
&(5)\end{aligned}$ & Type & $\begin{array}{l}m_{p g} \\
(7)\end{array}$ & $\begin{array}{c}a \\
, \\
(8)\end{array}$ & $\begin{array}{l}b \\
, \\
(9)\end{array}$ & $\begin{array}{l}\text { Dist } \\
\text { Mpc } \\
(10)\end{array}$ & Memb. & $\begin{array}{r}\theta \\
\operatorname{deg} \\
(12)\end{array}$ & $C_{31}(K)$ & $C_{31}(B)$ & notes \\
\hline 1885 & - & - & 124137.57 & 154933.2 & $\mathrm{Im}$ & 16.41 & 1.16 & 0.57 & 17 & $E$ & 4.32 & 2.80 & - & \\
\hline 1918 & - & - & 124218.10 & 54421.7 & $\mathrm{Im}$ & 15.80 & 1.03 & 0.36 & 17 & $\mathrm{~S}$ & 7.23 & 2.81 & - & \\
\hline 1929 & 7874 & 4633 & 124237.12 & 142122.0 & $\operatorname{Scd}(\mathrm{s})$ & 13.77 & 2.48 & 1.07 & 17 & E & 3.48 & 3.14 & 2.82 & \\
\hline 1932 & 7875 & 4634 & 124240.83 & 141746.0 & Sc (on edge) & 13.19 & 2.92 & 0.87 & 17 & E & 3.45 & 3.08 & 2.19 & \\
\hline 1952 & - & - & 124306.86 & 73858.4 & Im & 16.00 & 0.71 & 0.35 & 17 & E & 5.62 & 2.93 & - & \\
\hline 1970 & - & - & 124329.11 & 100534.7 & $\mathrm{Im}, \mathrm{N} ?$ & 15.80 & 0.71 & 0.50 & 17 & E & 3.86 & 2.94 & - & \\
\hline 1972 & 7896 & 4647 & 124332.28 & 113454.7 & $\mathrm{Sc}(\mathrm{rs})$ & 12.03 & 2.60 & 2.16 & 17 & E & 3.21 & 3.06 & - & * \\
\hline 1987 & 7902 & 4654 & 124356.71 & 130734.0 & $\mathrm{SBc}(\mathrm{rs})$ & 11.14 & 4.99 & 2.60 & 17 & E & 3.28 & 2.93 & 2.17 & \\
\hline 1992 & 7906 & - & 124410.02 & 120659.2 & $\mathrm{Im}$ & 15.50 & 0.81 & 0.51 & 17 & E & 3.27 & 2.80 & 3.16 & \\
\hline 1999 & 7915 & 4659 & 124429.38 & 132953.5 & $\mathrm{Sa}$ & 13.08 & 1.99 & 1.25 & 17 & E & 3.51 & 6.03 & - & \\
\hline 2006 & 7920 & 3718 & 124445.93 & 122111.7 & Amorphous & 13.68 & 2.60 & 0.71 & 17 & E & 3.40 & 3.19 & - & \\
\hline 2007 & - & 3716 & 124447.50 & 80629.7 & $\mathrm{Im} / \mathrm{BCD}$ : & 15.20 & 0.78 & 0.41 & 17 & E & 5.49 & 2.70 & - & \\
\hline 2023 & 7932 & 3742 & 124531.55 & 131951.3 & $\mathrm{SBc}(\mathrm{s})$ & 13.86 & 2.01 & 1.00 & 17 & E & 3.70 & 3.09 & - & \\
\hline 2033 & - & - & 124604.76 & 82830.8 & BCD & 14.65 & 0.73 & 0.73 & 17 & $\mathrm{E}$ & 5.42 & 3.70 & - & \\
\hline 2034 & - & - & 124607.96 & 100948.8 & Im & 15.82 & 0.78 & 0.52 & 17 & E & 4.36 & 2.46 & 9.72 & \\
\hline 2037 & - & - & 124615.15 & 101224.9 & $\operatorname{Im} / \mathrm{BCD}$ & 15.92 & 0.88 & 0.38 & 17 & E & 4.37 & 2.92 & 3.60 & \\
\hline 2058 & 7965 & 4689 & 124745.39 & 134548.3 & $\mathrm{Sc}(\mathrm{s})$ & 11.55 & 5.86 & 4.44 & 17 & E & 4.34 & 2.80 & 2.78 & \\
\hline 2066 & 7969 & 4694 & 124815.05 & 105906.7 & Amorphous & 12.19 & 3.20 & 1.16 & 17 & E & 4.49 & 4.21 & 6.88 & $*$ \\
\hline 2070 & 7970 & 4698 & 124822.96 & 82913.8 & $\mathrm{Sa}$ & 11.53 & 5.67 & 2.84 & 17 & E & 5.82 & 5.78 & 5.90 & $*$ \\
\hline 2087 & 7997 & 4733 & 125106.81 & 105444.3 & SB0/a & 12.63 & 1.96 & 1.96 & 17 & E & 5.18 & 2.73 & - & \\
\hline 2094 & - & - & 125235.75 & 102648.7 & Im: & 17.80 & 0.37 & 0.37 & 17 & E & 5.68 & - & - & \\
\hline
\end{tabular}

The Virgo serendipitous sample

\begin{tabular}{|c|c|c|c|c|c|c|c|c|c|c|c|c|c|c|}
\hline $\mathrm{VCC}$ & UGC & NGC/IC & $\begin{array}{c}\mathrm{RA}(2000) \\
\mathrm{h} \mathrm{m} \mathrm{s} \\
(4)\end{array}$ & $\begin{array}{ll} & \mathrm{dec} \\
o, & \prime \prime \\
& (5)\end{array}$ & Type & $\begin{array}{l}m_{p g} \\
\text { (7) }\end{array}$ & $\begin{array}{c}a \\
, \\
(8)\end{array}$ & $\begin{array}{l}b \\
, \\
(9)\end{array}$ & $\begin{array}{l}\text { Dist } \\
\text { Mpc } \\
\text { (10) }\end{array}$ & Memb. & $\begin{array}{r}\theta \\
\text { deg } \\
(12)\end{array}$ & $C_{31}(K)$ & $C_{31}(B)$ & notes \\
\hline 5 & - & - & 120833.15 & 150718.2 & $\mathrm{dE}$ & 18.90 & 0.39 & 0.25 & 32 & $\mathrm{M}$ & 6.06 & - & - & \\
\hline 79 & - & - & 121320.19 & 144819.1 & $\operatorname{Im} ?$ & 17.20 & 0.57 & 0.28 & 17 & $\mathrm{~N}$ & 4.89 & - & 1.75 & \\
\hline 90 & - & - & 121347.15 & 145013.2 & $\mathrm{dE}$ & 18.50 & 0.33 & 0.33 & 17 & $\mathrm{~N}$ & 4.81 & - & - & \\
\hline 147 & - & - & 121526.42 & 93543.6 & $\mathrm{dE}, \mathrm{N}$ & 19.00 & 0.25 & 0.25 & 17 & $\mathrm{~N}$ & 4.70 & - & - & \\
\hline 644 & - & - & 122329.26 & 173228.4 & $\mathrm{dE}$ & 20.00 & 0.28 & 0.28 & 17 & A & 5.45 & - & - & \\
\hline 823 & - & - & 122539.36 & 121853.4 & $\mathrm{dE}, \mathrm{N}$ & 16.06 & 0.84 & 0.84 & 17 & A & 1.27 & - & - & \\
\hline 832 & - & - & 122544.10 & 124029.4 & $\mathrm{dE}$ ? & 19.00 & 0.28 & 0.28 & 17 & A & 1.28 & - & 2.75 & \\
\hline 884 & - & - & 122615.21 & 130829.6 & $\mathrm{dE}:$ & 18.50 & 0.43 & 0.43 & 17 & A & 1.34 & - & - & \\
\hline 899 & - & - & 122626.24 & 64229.7 & Sm: & 16.60 & 0.57 & 0.28 & 56 & Bckg & 5.79 & - & - & \\
\hline 1018 & 7570 & 4432 & 122732.87 & 61354.3 & $\mathrm{Sc}(\mathrm{s})$ & 14.81 & 1.10 & 1.10 & 86.2 & Bckg & 6.21 & - & 2.87 & \\
\hline 1030 & 7575 & 4435 & 122740.42 & 130444.3 & SB0 & 11.84 & 2.92 & 2.48 & 17 & A & 1.03 & 10.06 & 7.07 & * \\
\hline 1146 & 7610 & 4458 & 122857.51 & 131431.0 & E & 12.93 & 1.80 & 1.52 & 17 & A & 0.96 & 7.99 & 7.65 & \\
\hline 1366 & - & - & 123131.30 & 113544.4 & $\mathrm{dE}, \mathrm{N}$ & 17.60 & 0.35 & 0.35 & 17 & A & 0.81 & - & - & \\
\hline 1385 & - & - & 123147.21 & 165014.6 & $\mathrm{dE} ?, \mathrm{~N}$ & 19.00 & 0.20 & 0.20 & 17 & A & 4.45 & - & - & \\
\hline 1821 & - & - & 124008.93 & 65302.1 & ? & 17.20 & 0.41 & 0.20 & 17 & $\mathrm{~S}$ & 5.97 & - & 1.90 & \\
\hline 1944 & - & - & 124251.62 & 141722.2 & BCD? & 18.00 & 0.46 & 0.22 & 17 & E & 3.49 & - & - & \\
\hline 1978 & 7898 & 4649 & 124339.58 & 113309.8 & So & 9.81 & 5.10 & 5.10 & 17 & E & 3.25 & 4.96 & - & * \\
\hline 2028 & - & - & 124537.25 & 131942.4 & dE: & 16.60 & 0.85 & 0.27 & 17 & E & 3.72 & - & - & \\
\hline
\end{tabular}

Other galaxies in the Virgo cluster

\begin{tabular}{|c|c|c|c|c|c|c|c|c|c|c|c|c|c|c|}
\hline VCC & $\begin{array}{l}\text { UGC } \\
\text { (2) }\end{array}$ & $\begin{array}{c}\text { NGC/IC } \\
\text { (3) }\end{array}$ & $\begin{array}{c}\mathrm{RA}(2000) \\
\mathrm{h} \mathrm{m} \mathrm{s} \\
(4)\end{array}$ & $\begin{array}{r}\mathrm{dec} \\
o^{\prime}, 11 \\
(5)\end{array}$ & $\begin{array}{l}\text { Type } \\
\text { (6) }\end{array}$ & $\begin{array}{l}m_{p g} \\
(7)\end{array}$ & $\begin{array}{l}a \\
, \\
(8)\end{array}$ & $\begin{array}{l}b \\
, \\
(9)\end{array}$ & $\begin{array}{l}\text { Dist } \\
\text { Mpc } \\
(10)\end{array}$ & Memb. & $\begin{array}{r}\theta \\
\text { deg } \\
(12)\end{array}$ & $C_{31}(K)$ & $C_{31}(B)$ & notes \\
\hline 1226 & 7629 & 4472 & 122946.74 & 80001.4 & $\mathrm{E} / \mathrm{S} 0$ & 9.31 & 10.25 & 8.11 & 17 & $\mathrm{~S}$ & 4.40 & 7.34 & 6.56 & * \\
\hline 1508 & 7709 & 4519 & 123330.18 & 83916.6 & $\mathrm{SBc}(\mathrm{rs})$ & 12.34 & 3.60 & 2.60 & 17 & $\mathrm{~S}$ & 3.79 & 3.17 & - & \\
\hline 1555 & 7727 & 4535 & 123420.32 & 81154.1 & $\mathrm{SBc}(\mathrm{s})$ & 10.51 & 8.33 & 7.43 & 17 & S & 4.28 & 2.18 & 2.60 & \\
\hline 1632 & 7760 & 4552 & 123539.87 & 123325.0 & So & 10.78 & 7.23 & 7.23 & 17 & A & 1.19 & 7.92 & - & $*$ \\
\hline 1939 & 7878 & 4636 & 124249.63 & 24118.1 & $\mathrm{E} / \mathrm{S} 0$ & 10.48 & 9.63 & 8.79 & 17 & $\mathrm{~S}$ & 10.15 & 10.44 & 5.77 & $*$ \\
\hline CGCG43041 & 7985 & 4713 & 124957.78 & 51839.2 & Sc & 12.3 & 3.20 & 2.16 & 17 & S & 8.51 & 2.93 & - & $*$ \\
\hline
\end{tabular}

Galaxies in the clusters A1367 and Coma

\begin{tabular}{|c|c|c|c|c|c|c|c|c|c|c|c|c|c|c|}
\hline CGCG & UGC & $\begin{array}{c}\text { NGC/IC } \\
\text { (3) }\end{array}$ & $\begin{array}{c}\mathrm{RA}(2000) \\
\text { h m s } \\
(4)\end{array}$ & $\begin{aligned} & \mathrm{dec} \\
o & , \quad 1 \\
& (5)\end{aligned}$ & $\begin{array}{l}\text { Type } \\
\text { (6) }\end{array}$ & $\begin{array}{l}m_{p g} \\
\text { (7) }\end{array}$ & $\begin{array}{c}a \\
, \\
(8)\end{array}$ & $\begin{array}{c}b \\
\text { (9) }\end{array}$ & $\begin{array}{l}\text { Dist } \\
\text { Mpc } \\
(10)\end{array}$ & Memb. & $\begin{array}{r}\theta \\
\text { deg } \\
(12)\end{array}$ & $C_{31}(K)$ & $C_{31}(B)$ & notes \\
\hline 97062 & - & - & 114214.64 & 195832.5 & $\mathrm{Pec}$ & 15.5 & 1.01 & 0.40 & 91.3 & A1367 & 0.53 & 3.16 & 2.93 & \\
\hline 97073 & - & - & 114256.43 & 195759.0 & Pec & 15.6 & 0.76 & 0.74 & 91.3 & A1367 & 0.37 & 2.74 & 2.13 & \\
\hline 97079 & - & - & 114313.35 & 200016.4 & $\mathrm{Pec}$ & 15.7 & 0.75 & 0.45 & 91.3 & A1367 & 0.33 & 2.88 & 2.11 & \\
\hline 97087 & 6697 & - & 114348.78 & 195809.7 & $\mathrm{Pec}$ & 14.3 & 2.00 & 0.50 & 91.3 & A1367 & 0.20 & 3.45 & 1.82 & * \\
\hline $97087 \mathrm{~N}$ & - & - & 114349.80 & 195834.0 & $\mathrm{Pec}$ & 17.2 & 0.20 & 0.15 & 91.3 & A1367 & 0.20 & - & - & \\
\hline 97138 & - & - & 114544.77 & 200151.1 & $\mathrm{Pec}$ & 15.5 & 0.75 & 0.64 & 91.3 & A1367 & 0.38 & 2.93 & - & \\
\hline 160020 & - & - & 125606.05 & 274039.9 & $\mathrm{Pec}$ & 15.5 & 0.45 & 0.22 & 96 & Coma & 0.90 & 2.13 & 2.97 & * \\
\hline 160026 & - & 3913 & 125628.56 & 271729.2 & $\mathrm{Sc}$ & 15.5 & 0.85 & 0.55 & 96 & Coma & 1.03 & 3.95 & 2.9 & \\
\hline 160055 & 8082 & 4848 & 125805.55 & 281432.8 & Sab & 14.2 & 1.51 & 0.58 & 96 & Coma & 0.48 & 4.12 & 2.96 & \\
\hline 160067 & - & - & 125837.17 & 271036.0 & $\mathrm{Pec}$ & 15.4 & 0.56 & 0.52 & 96 & Coma & 0.85 & 2.40 & - & \\
\hline 160076 & - & - & 125940.23 & 283751.2 & $\mathrm{Sc}$ & 15.6 & 0.64 & 0.60 & 96 & Coma & 0.65 & 3.25 & - & \\
\hline 160086 & - & - & 130033.58 & 273814.2 & $\mathrm{Pec}$ & 15.4 & 0.75 & 0.54 & 96 & Coma & 0.37 & 3.34 & - & $*$ \\
\hline 160088 & 8118 & 842 & 130039.63 & 290111.1 & $\mathrm{Sb}$ & 14.6 & 1.12 & 0.64 & 96 & Coma & 1.05 & 3.15 & 2.25 & \\
\hline 160095 & 8134 & 4921 & 130126.09 & 275308.1 & $\mathrm{Sb}$ & 13.7 & 2.28 & 2.23 & 96 & Coma & 0.35 & 3.46 & 2.83 & \\
\hline 160127 & - & - & 130426.46 & 271816.7 & $\mathrm{Sc}$ & 15.5 & 0.95 & 0.64 & 96 & Coma & 1.21 & 3.40 & - & \\
\hline 160128 & - & - & 130422.57 & 284838.5 & $\mathrm{Pec}$ & 15.3 & 0.63 & 0.62 & 96 & Coma & 1.29 & 3.00 & 2.38 & * \\
\hline 160139 & - & - & 130637.92 & 285059.1 & $\mathrm{Pec}$ & 15.0 & 1.22 & 0.64 & 96 & Coma & 1.71 & 2.73 & - & \\
\hline 160252 & - & 4040 & 130037.74 & 280328.2 & Pec & 15.1 & 0.85 & 0.36 & 96 & Coma & 0.18 & 3.26 & 2.22 & \\
\hline 160260 & 8128 & 4911 & 130056.10 & 274727.5 & $\mathrm{Sa}$ & 13.7 & 1.89 & 1.50 & 96 & Coma & 0.30 & 2.47 & 2.28 & \\
\hline
\end{tabular}


Table 1. continued.

\begin{tabular}{|c|c|c|c|c|c|c|c|c|c|c|c|c|c|c|}
\hline Dther gala & in th & Coma/A13 & supercluster & & & & & & & & & & & \\
\hline CGCG & $\overline{\mathrm{UGC}}$ & $\begin{array}{c}\text { NGC/IC } \\
\text { (3) }\end{array}$ & $\begin{array}{c}\mathrm{RA}(2000) \\
\mathrm{h} \text { m s } \\
(4)\end{array}$ & $\begin{array}{rl} & \mathrm{dec} \\
0 & 1 \\
0 & \quad 1 \\
& (5)\end{array}$ & $\begin{array}{l}\text { Type } \\
\text { (6) }\end{array}$ & $\begin{array}{r}m_{p g} \\
\text { (7) }\end{array}$ & $\begin{array}{l}a \\
{ }^{\prime} \\
(8)\end{array}$ & $\begin{array}{l}b \\
\prime \\
(9)\end{array}$ & $\begin{array}{r}\text { Dist } \\
\text { Mpc } \\
(10)\end{array}$ & Memb. & $\begin{array}{r}\theta \\
\text { deg } \\
(12)\end{array}$ & $C_{31}(K)$ & $C_{31}(B)$ & notes \\
\hline $159072 \mathrm{~N}$ & 7938 & 4676 & 124610.19 & 304354.7 & Pec(tides) & 14.8 & 2.20 & 0.40 & 88.4 & Pair & 4.07 & 3.53 & - & * \\
\hline $159072 \mathrm{~S}$ & 7939 & - & 124611.38 & 304325.0 & Pec(tides) & 14.8 & 1.70 & 0.70 & 87.9 & Pair & 4.06 & 3.62 & - & * \\
\hline 130023 & - & 860 & 131504.07 & 243701.3 & $\mathrm{Sa}$ & 14.8 & 0.96 & 0.61 & 54.1 & Group & 4.78 & - & 2.78 & $*$ \\
\hline
\end{tabular}

Notes on morphological type:

VCC 66: HII; VCC 92: M 98: HII and Seyfert; VCC 460: LINER; VCC 836: Seyfert2; VCC 857: LINER; VCC 1003: HII LINER; VCC 1030: LINER, tidally interacting with VCC 1043 (Arp 120); VCC 1043: LINER, tidally interacting with VCC 1030 (Arp 120); VCC 1110: LINER; VCC 1226: Seyfert2, M 49, Arp 134; VCC 1253: Seyfert 2; VCC 1632: LINER HII, M 89; VCC 1673: interacting with VCC 1676?; VCC 1676: interacting with VCC 1673?; VCC 1678: HII; VCC 1690: M 90: LINER, Seyfert; VCC 1727: M 58; LINER, Seyfert 1.9; VCC 1939: LINER; VCC 1972: interacting with VCC 1978 (M 60; Arp 116)?; VCC 1978: interacting with VCC 1972 (Arp 116)?; VCC 2066: HII; VCC 2070: Seyfert 2; CGCG 43041: LINER; CGCG 97087: merging system (Gavazzi et al. 2001); CGCG 130023: HII; CGCG 159072N,S: Arp 242 CGCG 160020: HII; CGCG 160086: starburst; CGCG 160128: HII. 
Table 2. The results of the observations.

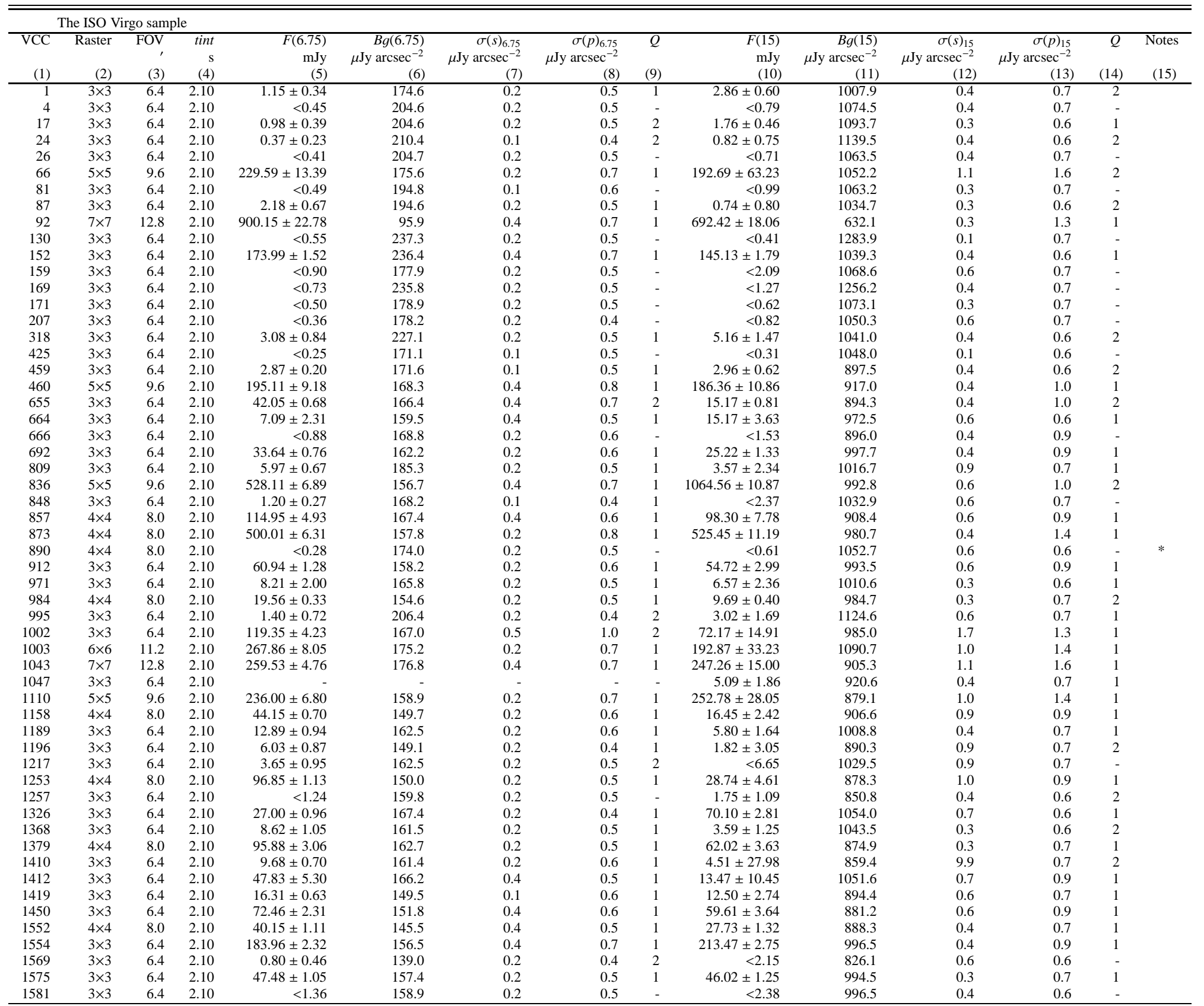


Table 2. continued.

\begin{tabular}{|c|c|c|c|c|c|c|c|c|c|c|c|c|c|c|}
\hline $\begin{array}{r}\mathrm{VCC} \\
\text { (1) }\end{array}$ & $\begin{array}{r}\text { Raster } \\
\text { (2) }\end{array}$ & $\begin{array}{r}\text { FOV } \\
\text { (3) }\end{array}$ & $\begin{aligned} \operatorname{tint} \\
\mathrm{s} \\
(4)\end{aligned}$ & $\begin{array}{r}F(6.75) \\
\text { mJy } \\
(5)\end{array}$ & $\begin{array}{r}B g(6.75) \\
\mu \mathrm{Jy} \operatorname{arcsec}^{-2} \\
(6)\end{array}$ & $\begin{array}{r}\sigma(s)_{6.75} \\
\mu \mathrm{Jy} \operatorname{arcsec}^{-2} \\
(7)\end{array}$ & $\begin{array}{r}\sigma(p)_{6.75} \\
\mu \mathrm{Jy} \operatorname{arcsec}^{-2} \\
(8)\end{array}$ & $\bar{Q}$ & $\begin{array}{r}F(15) \\
\text { mJy } \\
(10)\end{array}$ & $\begin{array}{r}B g(15) \\
\mu \mathrm{Jy} \operatorname{arcsec}^{-2} \\
(11)\end{array}$ & $\begin{array}{r}\sigma(s)_{15} \\
\mu \mathrm{Jy} \operatorname{arcsec} c^{-2} \\
(12)\end{array}$ & $\begin{array}{r}\sigma(p)_{15} \\
\mu \mathrm{Jy} \operatorname{arcsec}-{ }^{-2} \\
(13)\end{array}$ & $\begin{array}{r}Q \\
(14)\end{array}$ & $\begin{array}{l}\text { Notes } \\
\text { (15) }\end{array}$ \\
\hline $\begin{array}{r}111 \\
1596 \\
\end{array}$ & $\frac{(2)}{3 \times 3}$ & $\frac{(3)}{6.4}$ & $\frac{(4)}{2.10}$ & $\begin{array}{r}(3) \\
<0.21\end{array}$ & $\begin{array}{r}\text { (f) } \\
157.4\end{array}$ & $\begin{array}{r}\text { (t) } \\
\end{array}$ & $\begin{array}{r}(8) \\
0.4\end{array}$ & (9) & $\frac{(10)}{<0.62}$ & $\begin{array}{r}(11) \\
945.4\end{array}$ & $\begin{array}{r}(12) \\
0.4\end{array}$ & $\begin{array}{r}\text { (13) } \\
0.7\end{array}$ & (14) & \\
\hline 1673 & $5 \times 5$ & 9.6 & 2.10 & $324.33 \pm 6.48$ & 174.8 & 0.4 & 0.7 & 1 & $319.63 \pm 22.95$ & 973.2 & 1.3 & 1.8 & 1 & $*$ \\
\hline 1675 & $3 \times 3$ & 6.4 & 2.10 & $<1.66$ & 156.3 & 0.4 & 0.4 & & $<0.77$ & 975.1 & 0.1 & 0.7 & - & \\
\hline 1678 & $3 \times 3$ & 6.4 & 2.10 & $<3.48$ & 154.8 & 0.4 & 0.4 & 2 & $<10.93$ & 962.0 & 1.1 & 0.6 & & \\
\hline 1686 & $4 \times 4$ & 8.0 & 2.10 & $25.20 \pm 2.59$ & 143.9 & 0.4 & 0.7 & 2 & $19.42 \pm 4.07$ & 872.3 & 0.6 & 1.0 & 2 & * \\
\hline 1690 & $8 \times 8$ & 14.4 & 2.10 & $830.16 \pm 18.63$ & 144.2 & 0.4 & 0.7 & 1 & $972.71 \pm 36.73$ & 872.0 & 0.7 & 1.0 & 1 & \\
\hline 1699 & $3 \times 3$ & 6.4 & 2.10 & $4.15 \pm 0.74$ & 150.6 & 0.2 & 0.5 & 1 & $14.04 \pm 0.46$ & 991.1 & 0.1 & 0.6 & 1 & \\
\hline 1725 & $3 \times 3$ & 6.4 & 2.10 & $2.32 \pm 0.38$ & 150.9 & 0.1 & 0.4 & 2 & $2.70 \pm 0.46$ & 956.9 & 0.1 & 0.6 & 2 & \\
\hline 1750 & $3 \times 3$ & 6.4 & 2.10 & $0.28 \pm 0.10$ & 156.5 & 0.1 & 0.5 & 3 & $<0.93$ & 940.1 & 0.7 & 0.9 & - & \\
\hline 1757 & $3 \times 3$ & 6.4 & 2.10 & $<0.99$ & 142.2 & 0.1 & 0.4 & - & $<2.27$ & 867.9 & 0.3 & 0.7 & . & \\
\hline 1758 & $3 \times 3$ & 6.4 & 2.10 & $5.92 \pm 0.83$ & 152.9 & 0.2 & 0.4 & 1 & $0.98 \pm 0.99$ & 938.5 & 0.3 & 0.6 & 2 & \\
\hline 1784 & $3 \times 3$ & 6.4 & 2.10 & $<0.68$ & 152.8 & 0.2 & 0.5 & & $<2.69$ & 843.5 & 1.0 & 1.0 & 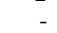 & \\
\hline 1789 & $3 \times 3$ & 6.4 & 2.10 & $1.40 \pm 0.26$ & 210.2 & 0.1 & 0.5 & 2 & $<2.78$ & 1192.2 & 0.7 & 0.9 & - & \\
\hline 1791 & $3 \times 3$ & 6.4 & 2.10 & $1.88 \pm 0.57$ & 151.9 & 0.2 & 0.4 & 2 & $2.97 \pm 0.37$ & 958.6 & 0.1 & 0.6 & 1 & \\
\hline 1804 & $3 \times 3$ & 6.4 & 2.10 & $0.41 \pm 0.17$ & 178.2 & 0.1 & 0.4 & 2 & $<3.62$ & 1020.4 & 1.4 & 0.9 & 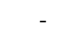 & \\
\hline 1811 & $3 \times 3$ & 6.4 & 2.10 & $74.49 \pm 2.83$ & 158.6 & 0.5 & 0.7 & 1 & $52.59 \pm 2.51$ & 877.3 & 0.4 & 0.7 & 1 & \\
\hline 1952 & $3 \times 3$ & 6.4 & 2.10 & $<0.61$ & 206.7 & 0.2 & 0.5 & . & $<1.05$ & 1010.8 & 0.4 & 0.6 & . & \\
\hline 1970 & $3 \times 3$ & 6.4 & 2.10 & $<0.61$ & 163.1 & 0.2 & 0.5 & - & $<2.74$ & 940.2 & 1.1 & 0.7 & - & \\
\hline 1972 & $6 \times 6$ & 11.2 & 2.10 & $501.09 \pm 2.34$ & 181.0 & 0.4 & 1.0 & 1 & $493.38 \pm 7.28$ & 846.4 & 1.1 & 1.6 & 1 & \\
\hline 1987 & $5 \times 5$ & 9.6 & 2.10 & $1051.86 \pm 27.70$ & 173.0 & 0.5 & 1.0 & 1 & $1101.42 \pm 32.80$ & 820.0 & 0.6 & 1.3 & 1 & \\
\hline 1992 & $3 \times 3$ & 6.4 & 2.10 & $0.58 \pm 0.18$ & 138.6 & 0.1 & 0.4 & 3 & $\begin{array}{l}<1.210 .61 \\
\end{array}$ & 829.1 & 0.4 & 0.7 & & \\
\hline 1999 & $3 \times 3$ & 6.4 & 2.10 & $10.06 \pm 1.04$ & 140.7 & 0.2 & 0.5 & 1 & $3.98 \pm 4.30$ & 811.4 & 1.0 & 2.0 & 2 & \\
\hline 2006 & $3 \times 3$ & 6.4 & 2.10 & $2.93 \pm 0.78$ & 170.3 & 0.1 & 0.4 & 1 & $1.49 \pm 1.83$ & 803.9 & 0.3 & 0.6 & 2 & \\
\hline 2007 & $3 \times 3$ & 6.4 & 2.10 & $1.80 \pm 0.33$ & 202.2 & 0.2 & 0.5 & 1 & $<2.28$ & 983.7 & 0.9 & 0.9 & - & \\
\hline 2023 & $3 \times 3$ & 6.4 & 2.10 & $5.46 \pm 1.05$ & 135.3 & 0.2 & 0.5 & 1 & $5.45 \pm 1.85$ & 786.1 & 0.4 & 0.6 & 1 & \\
\hline 2033 & $3 \times 3$ & 6.4 & 2.10 & $0.59 \pm 0.17$ & 196.5 & 0.1 & 0.4 & 2 & $1.17 \pm 0.88$ & 1097.2 & 0.7 & 0.6 & 3 & \\
\hline 2034 & $3 \times 3$ & 6.4 & 2.10 & $0.19 \pm 0.33$ & 164.4 & 0.2 & 0.4 & 3 & $<0.49$ & 926.7 & 0.1 & 0.7 & - & \\
\hline 2037 & $3 \times 3$ & 6.4 & 2.10 & $<0.75$ & 160.4 & 0.2 & 0.4 & 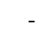 & $<1.72$ & 947.0 & 0.6 & 0.5 & - & \\
\hline 2058 & $5 \times 5$ & 9.6 & 2.10 & $316.50 \pm 9.16$ & 131.6 & 0.4 & 0.6 & 1 & $352.68 \pm 18.06$ & 797.9 & 0.7 & 1.0 & 1 & \\
\hline 2066 & $4 \times 4$ & 8.0 & 2.10 & $\begin{array}{r}68.00 \pm 0.64\end{array}$ & 182.2 & 0.2 & 0.5 & 1 & $68.13 \pm 2.96$ & 1009.8 & 1.1 & 0.7 & 1 & \\
\hline 2070 & $4 \times 4$ & 8.0 & 2.10 & $88.49 \pm 2.95$ & 185.7 & 0.2 & 0.5 & 1 & $66.58 \pm 8.69$ & 1026.9 & 0.7 & 1.0 & 1 & \\
\hline 2087 & $3 \times 3$ & 6.4 & 2.10 & $11.46 \pm 1.51$ & 183.3 & 0.4 & 0.5 & 1 & $7.06 \pm 1.21$ & 1011.2 & 0.3 & 0.7 & 1 & \\
\hline 2094 & $3 \times 3$ & 6.4 & 2.10 & $0.44 \pm 0.19$ & 185.9 & 0.2 & 0.5 & 3 & $<0.46$ & 1013.3 & 0.3 & 0.7 & - & \\
\hline
\end{tabular}


Table 2. continued.

\begin{tabular}{|c|c|c|c|c|c|c|c|c|c|c|c|c|c|c|}
\hline \multicolumn{15}{|c|}{ ipitous : } \\
\hline & & & timt & mJy & $\mu \mathrm{Jy} \operatorname{arcsec}^{-2}$ & $\mu \mathrm{Jy} \operatorname{arcsec}^{-2}$ & $\mu \mathrm{Jy} \operatorname{arcsec}^{-2}$ & 2 & $\mathrm{mJy}$ & $\mu \mathrm{Jy} \operatorname{arcsec}^{-2}$ & $\begin{array}{r}\sigma(s)_{15} \\
\mu \mathrm{Jy} \operatorname{arcsec}^{-2}\end{array}$ & $\begin{array}{r}\sigma(p)_{15} \\
\mu \mathrm{Jy} \operatorname{arcsec}^{-2}\end{array}$ & $Q$ & Notes \\
\hline (1) & (2) & (3) & (4) & (5) & (6) & (7) & (8) & (9) & (10) & (11) & (12) & (13) & (14) & (15) \\
\hline 5 & $3 \times 3$ & 6.4 & 2.10 & $<0.38$ & 204.6 & 0.2 & 0.5 & - & $<0.66$ & 1074.5 & 0.4 & 0.7 & & 4 \\
\hline 79 & $3 \times 3$ & 6.4 & 2.10 & $<0.33$ & 194.8 & 0.1 & 0.6 & - & $<0.62$ & 1063.2 & 0.3 & 0.7 & - & 81 \\
\hline 90 & $7 \times 7$ & 12.8 & 2.10 & $<0.52$ & 95.9 & 0.4 & 0.6 & - & $<0.51$ & 632.1 & 0.3 & 1.1 & - & 92 \\
\hline 147 & $3 \times 3$ & 6.4 & 2.10 & $<0.32$ & 236.4 & 0.2 & 0.6 & - & $<0.55$ & 1039.3 & 0.4 & 1.0 & - & 152 \\
\hline 644 & $3 \times 3$ & 6.4 & 2.10 & $<0.48$ & 166.4 & 0.2 & 0.7 & - & $<0.58$ & 900.8 & 0.4 & 1.0 & - & 655 \\
\hline 823 & $3 \times 3$ & 6.4 & 2.10 & $<0.73$ & 185.3 & 0.2 & 0.5 & - & $<2.46$ & 1016.7 & 0.9 & 0.9 & - & 809 \\
\hline 832 & $5 \times 5$ & 9.6 & 2.10 & $<0.48$ & 156.4 & 0.2 & 0.7 & - & $<0.73$ & 993.2 & 0.6 & 1.0 & - & 836 \\
\hline 884 & $4 \times 4$ & 8.0 & 2.10 & $<0.49$ & 157.8 & 0.2 & 0.7 & - & $<0.74$ & 981.0 & 0.4 & 1.0 & - & 873 \\
\hline 899 & $4 \times 4$ & 8.0 & 2.10 & $<0.50$ & 174.0 & 0.2 & 0.5 & - & $<1.13$ & 1052.7 & 0.6 & 0.6 & - & 890 \\
\hline 1018 & $3 \times 3$ & 6.4 & 2.10 & $7.68 \pm 0.74$ & 167.4 & 0.4 & 0.7 & 2 & $2.02 \pm 3.31$ & 986.1 & 1.4 & 1.0 & 1 & 1002 \\
\hline 1030 & $7 \times 7$ & 12.8 & 2.10 & $119.98 \pm 4.61$ & 177.3 & 0.4 & 0.7 & 1 & $76.48 \pm 8.73$ & 904.9 & 0.6 & 1.1 & 1 & 1043 \\
\hline 1146 & $4 \times 4$ & 8.0 & 2.10 & $9.67 \pm 0.91$ & 149.7 & 0.2 & 0.6 & 1 & $4.83 \pm 3.16$ & 906.6 & 0.9 & 0.9 & 1 & 1158 \\
\hline 1366 & $3 \times 3$ & 6.4 & 2.10 & $<0.38$ & 161.5 & 0.2 & 0.6 & - & $<0.44$ & 1043.5 & 0.3 & 0.7 & - & 1368 \\
\hline 1385 & $4 \times 4$ & 8.0 & 2.10 & $<0.27$ & 162.7 & 0.2 & 0.5 & - & $<0.34$ & 874.9 & 0.3 & 0.7 & - & 1379 \\
\hline 1821 & $3 \times 3$ & 6.4 & 2.10 & $<0.22$ & 151.9 & 0.1 & 0.4 & - & $<0.67$ & 960.3 & 0.4 & 0.6 & - & 1822 \\
\hline 1944 & $6 \times 6$ & 11.2 & 2.10 & $0.32 \pm 0.22$ & 140.4 & 0.2 & 0.6 & 3 & $<1.22$ & 827.4 & 0.7 & 1.0 & - & 1932 \\
\hline 1978 & $6 \times 6$ & 11.2 & 2.10 & $431.24 \pm 7.06$ & 181.0 & 0.4 & 1.0 & 1 & $171.69 \pm 22.18$ & 845.4 & 1.1 & 1.3 & 1 & 1972 \\
\hline 2028 & $3 \times 3$ & 6.4 & 2.10 & $<0.74$ & 135.3 & 0.2 & 0.5 & - & $<1.26$ & 786.1 & 0.4 & 0.6 & - & 2023 \\
\hline \multicolumn{15}{|c|}{ Other galaxies in the Virgo cluster } \\
\hline $\mathrm{VCC}$ & Raster & FOV & $\begin{array}{l}\text { tint } \\
\mathrm{s}\end{array}$ & $\begin{array}{l}F(6.75) \\
\mathrm{mJy}\end{array}$ & $\begin{array}{r}B g(6.75) \\
\mu \mathrm{Jy} \operatorname{arcsec}^{-2}\end{array}$ & $\begin{array}{r}\sigma(s)_{6.75} \\
\mu \mathrm{Jy} \operatorname{arcsec}^{-2}\end{array}$ & $\begin{array}{r}\sigma(p)_{6.75} \\
\mu \mathrm{Jy} \operatorname{arcsec}^{-2}\end{array}$ & $Q$ & $\begin{aligned} F(15) \\
\mathrm{mJy}\end{aligned}$ & $\begin{array}{r}B g(15) \\
\mu \mathrm{Jy} \operatorname{arcsec}^{-2}\end{array}$ & $\begin{array}{r}\sigma(s)_{15} \\
\mu \mathrm{Jy} \operatorname{arcsec}^{-2}\end{array}$ & $\begin{array}{r}\sigma(p)_{15} \\
\mu \mathrm{Jy} \operatorname{arcsec}^{-2}\end{array}$ & $Q$ & Notes \\
\hline (1) & (2) & (3) & (4) & (5) & & (7) alesec & poy alcsec (8) & (9) & $(10)$ & Hojy aresec (11) & (12) & AJy aicsec (13) & (14) & (15) \\
\hline 1226 & $5 \times 5$ & 1.8 & 5.04 & $31.17 \pm 41.41$ & 55.3 & 0.6 & 0.7 & 3 & $10.76 \pm 19.62$ & 259.8 & 0.3 & 0.6 & 3 & $*$ \\
\hline 1508 & $2 \times 2$ & 4.45 & 5.04 & $158.09 \pm 3.92$ & 183.2 & 0.4 & 1.0 & 2 & $201.81 \pm 10.76$ & 1049.5 & 1.0 & 1.8 & 2 & * \\
\hline 1555 & $4 \times 4$ & 8.0 & 5.04 & $1029.23 \pm 37.09$ & 169.4 & 0.4 & 0.8 & 1 & $853.78 \pm 102.39$ & 1043.4 & 1.0 & 1.8 & 1 & \\
\hline 1632 & $5 \times 3$ & $15.2 \times 3.6$ & 1.12 & $180.51 \pm 13.10$ & 175.0 & 0.4 & 0.8 & 2 & $39.31 \pm 25.82$ & 986.5 & 0.7 & 1.4 & 3 & * \\
\hline 1939 & $5 \times 5$ & 1.8 & 5.04 & $11.51 \pm 22.13$ & 70.0 & 0.4 & 0.5 & 3 & $3.49 \pm 26.18$ & 348.9 & 0.4 & 0.3 & 3 & * \\
\hline CGCG43041 & $2 \times 2$ & 4.45 & 5.04 & $189.20 \pm 8.53$ & 130.5 & 1.0 & 1.0 & 2 & $133.95 \pm 10.12$ & 847.5 & 1.1 & 1.7 & 2 & * \\
\hline \multicolumn{15}{|c|}{ Galaxies in the clusters A1367 and Coma } \\
\hline CGCG & Raster & FOV & $\begin{array}{r}\text { tint } \\
\mathrm{s}\end{array}$ & $\begin{array}{r}F(6.75) \\
\text { mJy }\end{array}$ & $\begin{array}{r}B g(6.75) \\
\mu \mathrm{Jy} \operatorname{arcsec}^{-2}\end{array}$ & $\begin{array}{r}\sigma(s)_{6.75} \\
\mu \mathrm{Jy} \operatorname{arcsec}^{-2}\end{array}$ & $\begin{array}{r}\sigma(p)_{6.75} \\
\mu \mathrm{Jy} \operatorname{arcsec}^{-2}\end{array}$ & $\bar{Q}$ & $\begin{array}{r}F(15) \\
\text { mJy }\end{array}$ & $\begin{array}{r}B g(15) \\
\mu \mathrm{Jy} \operatorname{arcsec}^{-2}\end{array}$ & $\begin{array}{r}\sigma(s)_{15} \\
\mu \mathrm{Jy} \operatorname{arcsec}^{-2}\end{array}$ & $\begin{array}{r}\sigma(p)_{15} \\
\mu \mathrm{Jy} \operatorname{arcsec}^{-2}\end{array}$ & $\bar{Q}$ & Notes \\
\hline (1) & (2) & (3) & (4) & (5) & (6) & (7) & (8) & (9) & $(10)$ & $(11)$ & (12) & (13) & (14) & (15) \\
\hline 97062 & $2 \times 2$ & 4.2 & 5.04 & $9.60 \pm 0.48$ & 171.3 & 0.2 & 0.2 & 1 & $7.20 \pm 0.86$ & 916.7 & 0.4 & 0.4 & 1 & \\
\hline 97073 & $2 \times 2$ & 4.2 & 5.04 & $5.78 \pm 0.25$ & 170.9 & 0.2 & 0.2 & 1 & $2.74 \pm 0.44$ & 911.1 & 0.4 & 0.3 & 1 & \\
\hline 97079 & $2 \times 2$ & 4.2 & 5.04 & $6.40 \pm 0.25$ & 171.2 & 0.2 & 0.2 & 1 & $7.48 \pm 0.44$ & 924.0 & 0.4 & 0.4 & 1 & \\
\hline 97087 & $2 \times 2$ & 4.2 & 5.04 & $50.83 \pm 3.30$ & 171.2 & 0.4 & 0.4 & 1 & $52.40 \pm 6.50$ & 919.8 & 0.7 & 0.4 & 1 & \\
\hline $97087 \mathrm{~N}$ & $2 \times 2$ & 4.2 & 5.04 & $2.92 \pm 0.10$ & 171.2 & 0.2 & 0.4 & 2 & $2.09 \pm 0.16$ & 919.8 & 0.4 & 0.4 & 1 & * \\
\hline 97138 & $2 \times 2$ & 4.2 & 5.04 & $1.62 \pm 0.09$ & 167.3 & 0.1 & 0.2 & 1 & $1.08 \pm 0.37$ & 904.9 & 0.6 & 0.4 & 2 & \\
\hline 160020 & $2 \times 2$ & 4.2 & 5.04 & $15.52 \pm 0.36$ & 88.8 & 0.2 & 0.4 & 1 & $17.27 \pm 0.44$ & 528.2 & 0.3 & 0.6 & 1 & \\
\hline 160026 & $2 \times 2$ & 4.2 & 5.04 & $7.89 \pm 0.36$ & $\begin{array}{l}89.6 \\
89.7\end{array}$ & 0.2 & 0.2 & 1 & $6.90 \pm 0.44$ & 542.8 & 0.3 & 0.6 & 1 & \\
\hline 160055 & $2 \times 2$ & 4.2 & 5.04 & $41.77 \pm 0.80$ & 87.0 & 0.2 & 0.4 & 1 & $42.20 \pm 0.95$ & 513.7 & 0.3 & 0.6 & 1 & \\
\hline 160067 & $2 \times 2$ & 4.2 & 5.04 & $14.30 \pm 0.36$ & 100.0 & 0.2 & 0.2 & 1 & $13.41 \pm 0.43$ & 557.7 & 0.3 & 0.4 & 1 & \\
\hline 160076 & $2 \times 2$ & 4.2 & 5.04 & $2.14 \pm 0.36$ & 86.2 & 0.2 & 0.2 & 1 & $1.73 \pm 0.43$ & 558.0 & 0.3 & 0.4 & 1 & \\
\hline 160086 & $2 \times 2$ & 4.2 & 5.04 & $9.18 \pm 0.36$ & 86.2 & 0.2 & 0.4 & 1 & $10.87 \pm 0.44$ & 525.1 & 0.3 & 0.6 & 1 & \\
\hline 160088 & $2 \times 2$ & 4.2 & 5.04 & $19.40 \pm 1.19$ & 88. & 0.2 & 0.4 & 1 & $12.22 \pm 1.42$ & 520.8 & 0.3 & 0.6 & 1 & \\
\hline 160095 & $2 \times 2$ & 4.2 & 5.04 & $14.08 \pm 1.92$ & 88 & 0.2 & 0.4 & 1 & $9.22 \pm 2.27$ & 529.2 & 0.3 & 0.4 & 1 & \\
\hline 160127 & $2 \times 2$ & 4.2 & 5.04 & $3.02 \pm 0.25$ & 84.4 & 0.2 & 0.4 & 1 & $3.42 \pm 0.30$ & 503.4 & 0.3 & 0.4 & 1 & \\
\hline 160128 & $2 \times 2$ & 4.2 & 5.04 & $4.82 \pm 0.36$ & 83.8 & 0.2 & 0.2 & 1 & $4.59 \pm 0.22$ & 505.4 & 0.1 & 0.3 & 1 & \\
\hline 160139 & $2 \times 2$ & 4.2 & 5.04 & $3.07 \pm 0.63$ & 81.8 & 0.2 & 0.2 & 1 & $3.39 \pm 0.38$ & 502.6 & 0.1 & 0.3 & 1 & \\
\hline 160252 & $2 \times 2$ & 4.2 & 5.04 & $36.86 \pm 0.79$ & 86.7 & 0.2 & 0.2 & 1 & $37.10 \pm 0.95$ & 517.9 & 0.3 & 0.4 & 1 & \\
\hline 160260 & $2 \times 2$ & 4.2 & 5.04 & $67.75 \pm 1.19$ & 89.2 & 0.2 & 0.4 & 1 & $68.45 \pm 1.41$ & 530.0 & 0.3 & 0.4 & 1 & \\
\hline
\end{tabular}


Table 2. continued.

\begin{tabular}{|c|c|c|c|c|c|c|c|c|c|c|c|c|c|c|}
\hline VCC & Raster & FOV & $\begin{array}{r}\text { tint } \\
\mathrm{s}\end{array}$ & $\begin{array}{r}F(6.75) \\
\mathrm{mJy}\end{array}$ & $\begin{array}{r}B g(6.75) \\
\mu \mathrm{Jy} \operatorname{arcsec}^{-2}\end{array}$ & $\begin{array}{r}\sigma(s)_{6.75} \\
\mu \mathrm{Jy} \operatorname{arcsec}^{-2}\end{array}$ & $\begin{array}{r}\sigma(p)_{6.75} \\
\mu \mathrm{Jy} \operatorname{arcsec}^{-2}\end{array}$ & $\bar{Q}$ & $\begin{aligned} \begin{array}{l}F(15) \\
\text { mJy }\end{array} & \end{aligned}$ & $\begin{array}{r}B g(15) \\
\mu \mathrm{Jy} \operatorname{arcsec}^{-2}\end{array}$ & $\begin{array}{r}\sigma(s)_{15} \\
\mu \mathrm{Jy} \operatorname{arcsec}^{-2}\end{array}$ & $\begin{array}{r}\sigma(p)_{15} \\
\mu \mathrm{Jy} \operatorname{arcsec}^{-2}\end{array}$ & $\bar{Q}$ & Notes \\
\hline (1) & (2) & (3) & (4) & (5) & (6) & (7) & (8) & (9) & (10) & (11) & (12) & (13) & (14) & (15) \\
\hline $159072 \mathrm{~N}$ & $2 \times 2$ & 2.1 & 2.1 & $65.54 \pm 2.97$ & 35.4 & 0.6 & 0.5 & 1 & $68.82 \pm 5.62$ & 166.5 & 1.1 & 0.7 & 1 & * \\
\hline $159072 \mathrm{~S}$ & $2 \times 2$ & 2.1 & 2.1 & $11.23 \pm 2.05$ & 35.4 & 0.6 & 0.5 & 1 & $11.96 \pm 3.87$ & 166.5 & 1.1 & 0.7 & 1 & * \\
\hline 130023 & $2 \times 2$ & 4.45 & 5.04 & $28.69 \pm 0.80$ & 113.9 & 0.5 & 0.5 & 2 & $63.58 \pm 1.42$ & 602.6 & 0.9 & 0.9 & 2 & * \\
\hline
\end{tabular}

Notes:

$\sigma(s)_{\lambda}$ and $\sigma(p)_{\lambda}$ are given in units of mJy per $6^{\prime \prime} \times 6^{\prime \prime}$ pixel.

VCC 890: in the same frame of VCC 899.

VCC 1508: at $15 \mu \mathrm{m}$ tint $=2.1 \mathrm{~s}$.

VCC 1632: at $15 \mu \mathrm{m}$ tint $=2.1 \mathrm{~s}$.

VCC 1673: in the same frame of VCC 1676

VCC 1676: in the same frame of VCC 1673

VCC 1686: the given value is the average value obtained from the pointed observation of VCC 1686 and that extracted from the image of the nearby VCC 1690

VCC 1929: in the same frame of VCC 1932

VCC 1932: in the same frame of VCC 1929

VCC 1226: images taken with a pixel size of 3 arcsec.

VCC 1939: images taken with a pixel size of 3 arcsec.

CGCG 43041: at $15 \mu \mathrm{m}$ tint $=2.1 \mathrm{~s}$.

CGCG 97087N: serendipitously detected in the frame of CGCG 97087.

CGCG $159072 \mathrm{~N}$ and S: in the same frame, obtained with a pixel size of 3 arcsec.

CGCG 130023: at $15 \mu \mathrm{m}$ tint $=2.1 \mathrm{~s}$. 
A. Boselli et al.: Mid-IR emission of galaxies in the Virgo cluster. III., Online Material p 9

Table 4. Parameters of the resolved galaxies.

\begin{tabular}{|c|c|c|c|c|c|c|c|c|}
\hline$\overline{\mathrm{VCC}}$ & $\begin{array}{r}C_{31}(6.75) \\
(2)\end{array}$ & $\begin{array}{r}r_{5}(6.75) \\
, \\
(3)\end{array}$ & $\begin{array}{r}R_{\mathrm{e}}(6.75) \\
\text { (4) }\end{array}$ & $\begin{array}{r}\mu_{\mathrm{e}}(6.75) \\
\mu \mathrm{Jy} \operatorname{arcsec}^{-2} \\
(5)\end{array}$ & $\begin{array}{r}C_{31}(15) \\
(6)\end{array}$ & $\begin{array}{r}r_{5}(15) \\
, \\
(7)\end{array}$ & $\begin{array}{r}R_{\mathrm{e}}(15) \\
, \\
(8)\end{array}$ & $\begin{array}{r}\mu_{\mathrm{e}}(15) \\
\mu \mathrm{Jy} \operatorname{arcsec}^{-2} \\
(9)\end{array}$ \\
\hline 66 & 3.02 & 111 & 78 & 16 & 2.58 & 104 & 78 & 18 \\
\hline 92 & 3.85 & - & 90 & 46 & 2.28 & 194 & 112 & 40 \\
\hline 460 & 7.26 & 75 & 34 & 66 & 5.16 & 54 & 13 & 323 \\
\hline 655 & 1.98 & 20 & 12 & 39 & 1.87 & 19 & 13 & 20 \\
\hline 692 & 2.99 & 26 & 15 & 34 & 2.65 & 21 & 11 & 31 \\
\hline 836 & 4.91 & 65 & 21 & 422 & 4.21 & 66 & 11 & 2117 \\
\hline 857 & 3.19 & 55 & 91 & 6 & 3.35 & 54 & 114 & 05 \\
\hline 873 & 2.84 & 76 & 43 & 126 & 2.90 & 76 & 41 & 120 \\
\hline 912 & 3.20 & 34 & 15 & 56 & 5.26 & 28 & 18 & 44 \\
\hline 984 & 5.48 & 17 & 50 & 4 & 2.98 & - & 43 & 1 \\
\hline 1002 & 1.73 & 44 & 34 & 18 & 1.69 & 43 & 36 & 12 \\
\hline 1003 & 7.07 & 86 & 48 & 40 & 11.45 & 46 & 72 & 15 \\
\hline 1043 & 5.13 & 54 & 24 & 121 & 4.75 & 53 & 21 & 106 \\
\hline 1110 & 3.19 & 79 & 56 & 22 & 2.99 & 90 & 60 & 21 \\
\hline 1158 & 6.94 & 25 & 20 & 41 & 2.99 & 13 & 171 & 1 \\
\hline 1253 & 5.01 & 32 & 132 & 3 & 4.71 & 18 & 16 & 21 \\
\hline 1379 & 1.80 & 53 & 43 & 18 & 2.12 & 44 & 39 & 14 \\
\hline 1552 & 3.53 & 26 & 28 & 10 & 3.21 & 24 & 23 & 11 \\
\hline 1554 & 2.75 & - & 24 & 117 & 2.82 & - & 21 & 159 \\
\hline 1555 & 2.95 & 151 & 102 & 38 & 2.51 & 142 & 88 & 49 \\
\hline 1673 & 1.91 & 63 & 31 & 70 & 2.57 & - & 27 & 87 \\
\hline 1676 & 3.57 & - & 37 & 231 & 3.72 & - & 35 & 279 \\
\hline 1690 & 3.36 & 107 & 64 & 60 & 5.12 & 96 & 61 & 70 \\
\hline 1727 & 2.80 & 107 & 77 & 25 & 3.13 & 109 & 68 & 31 \\
\hline 1811 & 2.74 & 36 & 19 & 31 & 2.85 & 34 & 19 & 28 \\
\hline 1813 & 5.34 & 54 & 36 & 8 & 8.23 & 23 & 47 & 45 \\
\hline 1929 & 3.20 & 28 & 23 & 15 & 2.81 & 29 & 19 & 18 \\
\hline 1932 & 2.67 & 55 & 26 & 131 & 2.60 & 58 & 26 & 152 \\
\hline 1972 & 2.67 & - & 33 & 92 & 2.78 & 74 & 31 & 96 \\
\hline 1978 & 5.27 & - & 72 & 27 & 6.71 & - & 77 & 10 \\
\hline 1987 & 2.41 & 113 & 56 & 104 & 2.62 & 115 & 57 & 99 \\
\hline 2058 & 2.39 & 75 & 37 & 51 & 2.43 & 78 & 38 & 50 \\
\hline 2066 & 2.44 & 24 & 10 & 122 & 2.34 & 23 & 10 & 112 \\
\hline 2070 & 5.30 & 52 & 65 & 8 & 2.67 & 53 & 170 & 3 \\
\hline
\end{tabular}


A. Boselli et al.: Mid-IR emission of galaxies in the Virgo cluster. III., Online Material p 10

Table 5. Supplementary parameters of the observations.

\begin{tabular}{|c|c|c|c|}
\hline \multicolumn{4}{|c|}{ The ISO Virgo sample } \\
\hline $\begin{array}{l}\text { Target name } \\
\text { (1) }\end{array}$ & $\begin{array}{l}\text { Filter } \\
(2)\end{array}$ & $\begin{array}{l}\text { ISO TDT number } \\
\text { (3) }\end{array}$ & $\begin{array}{r}\text { Raster orientation } \\
\text { (4) }\end{array}$ \\
\hline VCC 1 & LW2,LW3 & 22600301 & 21.2600 \\
\hline $\operatorname{VCC} 4$ & LW2 & 23501217 & 18.5900 \\
\hline VCC 4 & LW3 & 23501149 & 18.6000 \\
\hline VCC 17 & LW2 & 23501218 & 18.9000 \\
\hline VCC 17 & LW3 & 23501150 & 18.9100 \\
\hline VCC 24 & LW2,LW3 & 23501902 & 19.7500 \\
\hline VCC 26 & LW2 & 23501216 & 18.8300 \\
\hline VCC 26 & LW3 & 23501148 & 18.8400 \\
\hline VCC 66 & LW2,LW3 & 22701301 & 21.6700 \\
\hline VCC 81 & LW2 & 23501219 & 18.8800 \\
\hline VCC 81 & LW3 & 23501151 & 18.8900 \\
\hline VCC 87 & LW2 & 23501220 & 18.6600 \\
\hline VCC 87 & LW3 & 23501152 & 18.6700 \\
\hline VCC 92 & LW2,LW3 & 19900303 & 28.0100 \\
\hline VCC 130 & LW2 & 23901022 & 19.7500 \\
\hline VCC 130 & LW3 & 23900954 & 19.7500 \\
\hline VCC 152 & LW2 & 23900808 & 19.8200 \\
\hline VCC 152 & LW3 & 22700934 & 21.9800 \\
\hline VCC 159 & LW2 & 22802524 & 22.0000 \\
\hline VCC 159 & LW3 & 22802456 & 22.0100 \\
\hline VCC 169 & LW2 & 23901021 & 19.8000 \\
\hline VCC 169 & LW3 & 23900953 & 19.8000 \\
\hline VCC 171 & LW2 & 22802525 & 21.9900 \\
\hline VCC 171 & LW3 & 22802457 & 22.0000 \\
\hline VCC 207 & LW2 & 22802523 & 22.0600 \\
\hline VCC 207 & LW3 & 22802455 & 22.0700 \\
\hline VCC 318 & LW2 & 23900809 & 20.1100 \\
\hline VCC 318 & LW3 & 22700935 & 22.1800 \\
\hline VCC 425 & LW2 & 22802526 & 22.0100 \\
\hline VCC 425 & LW3 & 22802458 & 22.0200 \\
\hline VCC 459 & LW2,LW3 & 23401803 & 18.6100 \\
\hline VCC 460 & LW2 & 23401976 & 18.3700 \\
\hline VCC 460 & LW3 & 23500583 & 18.1200 \\
\hline VCC 655 & LW2 & 23401910 & 18.7600 \\
\hline VCC 655 & LW3 & 23500536 & 18.5100 \\
\hline VCC 664 & LW2 & 22701411 & 21.7800 \\
\hline VCC 664 & LW3 & 23100537 & 20.8900 \\
\hline VCC 666 & LW2,LW3 & 23402404 & 18.9000 \\
\hline VCC 692 & LW2 & 22701412 & 21.8300 \\
\hline VCC 692 & LW3 & 23100538 & 20.9500 \\
\hline VCC 809 & LW2,LW3 & 23502005 & 19.9800 \\
\hline VCC 836 & LW2 & 22701477 & 21.8300 \\
\hline VCC 836 & LW3 & 23100584 & 20.9200 \\
\hline VCC 848 & LW2,LW3 & 22801006 & 22.5600 \\
\hline VCC 857 & LW2 & 23401960 & 18.6800 \\
\hline VCC 857 & LW3 & 23500568 & 18.4200 \\
\hline VCC 873 & LW2 & 22701461 & 21.7800 \\
\hline VCC 873 & LW3 & 23100569 & 20.8300 \\
\hline VCC 890/9 & LW2,LW3 & 22801514 & 22.4600 \\
\hline VCC 912 & LW2 & 22701413 & 21.8700 \\
\hline VCC 912 & LW3 & 23100539 & 20.9500 \\
\hline VCC 971 & LW2 & 22801315 & 22.5700 \\
\hline VCC 971 & LW3 & 22701141 & 22.6800 \\
\hline VCC 984 & LW2 & 22701462 & 21.8800 \\
\hline VCC 984 & LW3 & 23100570 & 20.9500 \\
\hline VCC 995 & LW2,LW3 & 23901207 & 19.5500 \\
\hline VCC 1002 & LW2 & 22801314 & 22.5300 \\
\hline VCC 1002 & LW3 & 22701140 & 22.6500 \\
\hline VCC 1003 & LW2 & 23100990 & 21.2100 \\
\hline VCC 1003 & LW3 & 23600292 & 20.1500 \\
\hline VCC 1043 & LW2 & 23501494 & 19.8900 \\
\hline VCC 1043 & LW3 & 22600995 & 22.1500 \\
\hline VCC 1047 & LW2 & 23501421 & 20.0700 \\
\hline VCC 1047 & LW3 & 22600947 & 22.2300 \\
\hline VCC 1110 & LW2 & 23402178 & 19.1100 \\
\hline VCC 1110 & LW3 & 23402285 & 19.1000 \\
\hline VCC 1158 & LW2 & 22600465 & 22.2300 \\
\hline VCC 1158 & LW3 & 22600573 & 22.2200 \\
\hline VCC 1189 & LW2 & 22801316 & 22.5100 \\
\hline VCC 1189 & LW3 & 22701142 & 22.6400 \\
\hline VCC 1196 & LW2 & 22600422 & 22.1500 \\
\hline VCC 1196 & LW3 & 22600548 & 22.1400 \\
\hline VCC 1217 & LW2 & 23100917 & 21.2200 \\
\hline VCC 1217 & LW3 & 23600243 & 20.1300 \\
\hline VCC 1253 & LW2 & 22600464 & 22.2300 \\
\hline VCC 1253 & LW3 & 22600572 & 22.2200 \\
\hline VCC 1257 & LW2,LW3 & 23402008 & 19.1200 \\
\hline VCC 1326 & LW2 & 23100918 & 21.2400 \\
\hline VCC 1326 & LW3 & 23600244 & 20.1400 \\
\hline
\end{tabular}


A. Boselli et al.: Mid-IR emission of galaxies in the Virgo cluster. III., Online Material p 11

Table 5. continued.

\begin{tabular}{|c|c|c|c|}
\hline $\begin{array}{l}\text { Target name } \\
\text { (1) }\end{array}$ & $\begin{array}{l}\text { Filter } \\
\text { (2) }\end{array}$ & $\begin{array}{l}\text { ISO TDT number } \\
\text { (3) }\end{array}$ & $\begin{array}{r}\text { Raster orientation } \\
\text { (4) }\end{array}$ \\
\hline VCC 1368 & LW2 & 23100919 & 21.2400 \\
\hline VCC 1368 & LW3 & 23600245 & 20.1200 \\
\hline VCC 1379 & LW2 & 23402163 & 19.3300 \\
\hline VCC 1379 & LW3 & 23402271 & 19.3200 \\
\hline VCC 1410 & LW2,LW3 & 23402509 & 19.3400 \\
\hline VCC 1412 & LW2 & 23100920 & 21.3300 \\
\hline VCC 1412 & LW3 & 23600246 & 20.2400 \\
\hline VCC 1419 & LW2 & 22600423 & 22.3500 \\
\hline VCC 1419 & LW3 & 22600549 & 22.3400 \\
\hline VCC 1450 & LW2 & 22600424 & 22.3000 \\
\hline VCC 1450 & LW3 & 22600550 & 22.3000 \\
\hline VCC 1552 & LW2 & 22601467 & 22.3700 \\
\hline VCC 1552 & LW3 & 22600675 & 22.4600 \\
\hline VCC 1554 & LW2 & 22701227 & 22.7700 \\
\hline VCC 1554 & LW3 & 22801453 & 22.6300 \\
\hline VCC 1569 & LW2,LW3 & 22601110 & 22.3600 \\
\hline VCC 1575 & LW2 & 22701228 & 22.7200 \\
\hline VCC 1575 & LW3 & 22801454 & 22.5800 \\
\hline VCC 1581 & LW2 & 22802028 & 22.6300 \\
\hline VCC 1581 & LW3 & 22801960 & 22.6300 \\
\hline VCC 1596 & LW2,LW3 & 22802633 & 22.4500 \\
\hline VCC 1673/6 & LW2,LW3 & 23501602 & 20.5600 \\
\hline VCC 1675 & LW2 & 22802034 & 22.5200 \\
\hline VCC 1675 & LW3 & 22801966 & 22.5200 \\
\hline VCC 1678 & LW2 & 22701226 & 22.8100 \\
\hline VCC 1678 & LW3 & 22801452 & 22.6600 \\
\hline VCC 1686 & LW2 & 22601466 & 22.4700 \\
\hline VCC 1686 & LW3 & 22600674 & 22.5700 \\
\hline VCC 1690 & LW2,LW3 & 22600704 & 22.5700 \\
\hline VCC 1699 & LW2 & 22701229 & 22.7900 \\
\hline VCC 1699 & LW3 & 22801455 & 22.6500 \\
\hline VCC 1725 & LW2 & 22802032 & 22.5100 \\
\hline VCC 1725 & LW3 & 22801964 & 22.5100 \\
\hline VCC 1726 & LW2 & 22802027 & 22.6300 \\
\hline VCC 1726 & LW3 & 22801959 & 22.6300 \\
\hline VCC 1727 & LW2,LW3 & 23501305 & 20.5000 \\
\hline VCC 1730 & LW2 & 22701230 & 22.9000 \\
\hline VCC 1730 & LW3 & 22801456 & 22.7700 \\
\hline VCC 1750 & LW2 & 22801629 & 22.6600 \\
\hline VCC 1750 & LW3 & 22801861 & 22.6600 \\
\hline VCC 1757 & LW2 & 22601425 & 22.5300 \\
\hline VCC 1757 & LW3 & 22600651 & 22.6200 \\
\hline VCC 1758 & LW2 & 22801630 & 22.6000 \\
\hline VCC 1758 & LW3 & 22801862 & 22.5900 \\
\hline VCC 1784 & LW2 & 23402736 & 19.9300 \\
\hline VCC 1784 & LW3 & 23500768 & 19.7600 \\
\hline VCC 1789 & LW2 & 24100938 & 20.9300 \\
\hline VCC 1789 & LW3 & 24101070 & 20.9300 \\
\hline VCC 1791 & LW2 & 22802035 & 22.6000 \\
\hline VCC 1791 & LW3 & 22801967 & 22.6000 \\
\hline VCC 1804 & LW2,LW3 & 23600411 & 20.8200 \\
\hline VCC 1811 & LW2,LW3 & 23500807 & 19.8600 \\
\hline VCC 1813 & LW2 & 23901179 & 20.0100 \\
\hline VCC 1813 & LW3 & 23901179 & 20.0100 \\
\hline VCC 1822 & LW2 & 22801631 & 22.7200 \\
\hline VCC 1822 & LW3 & 22801863 & 22.7100 \\
\hline VCC 1869 & LW2 & 23500980 & 19.9500 \\
\hline VCC 1869 & LW3 & 23500980 & 19.9500 \\
\hline VCC 1885 & LW2 & 23402737 & 20.0000 \\
\hline VCC 1885 & LW3 & 23500769 & 19.8300 \\
\hline VCC 1918 & LW2 & 24100939 & 20.7100 \\
\hline VCC 1918 & LW3 & 24101071 & 20.7100 \\
\hline VCC $1929 / 32$ & LW2 & 22600206 & 22.8300 \\
\hline VCC $1929 / 32$ & LW3 & 22600206 & 22.8300 \\
\hline VCC 1952 & LW2 & 24300141 & 19.9000 \\
\hline VCC 1952 & LW3 & 23503173 & 21.3600 \\
\hline VCC 1970 & LW2 & 23503042 & 20.9300 \\
\hline VCC 1970 & LW3 & 23502974 & 20.9300 \\
\hline VCC 1972 & LW2 & 24101391 & 19.1000 \\
\hline VCC 1972 & LW3 & 22601693 & 22.9000 \\
\hline VCC 1987 & LW2 & 24101381 & 18.6400 \\
\hline VCC 1987 & LW3 & 22601688 & 22.8200 \\
\hline VCC 1992 & LW2,LW3 & 22601745 & 22.8900 \\
\hline VCC 1999 & LW2 & 22601333 & 22.8400 \\
\hline VCC 1999 & LW3 & 22601259 & 22.8500 \\
\hline
\end{tabular}


A. Boselli et al.: Mid-IR emission of galaxies in the Virgo cluster. III., Online Material p 12

Table 5. continued.

\begin{tabular}{llcr}
\hline $\begin{array}{l}\text { Target name } \\
(1)\end{array}$ & $\begin{array}{l}\text { Filter } \\
(2)\end{array}$ & $\begin{array}{c}\text { ISO TDT number } \\
(3)\end{array}$ & $\begin{array}{r}\text { Raster orientation } \\
(4)\end{array}$ \\
\hline VCC 2006 & LW2 & 24101331 & 18.9000 \\
VCC 2006 & LW3 & 22601657 & 22.9100 \\
VCC 2007 & LW2 & 2430140 & 19.7900 \\
VCC 2007 & LW3 & 23503172 & 21.3100 \\
VCC 2023 & LW2 & 22601332 & 22.9100 \\
VCC 2023 & LW3 & 22601258 & 22.9200 \\
VCC 2033 & LW2,LW3 & 24300212 & 19.6900 \\
VCC 2034 & LW2 & 23503043 & 20.9900 \\
VCC 2034 & LW3 & 23502975 & 20.9900 \\
VCC 2037 & LW2 & 23503044 & 20.9800 \\
VCC 2037 & LW3 & 23502976 & 20.9900 \\
VCC 2058 & LW2 & 22601382 & 23.0000 \\
VCC 2058 & LW3 & 22601289 & 23.0100 \\
VCC 2066 & LW2 & 24300377 & 19.0000 \\
VCC 2066 & LW3 & 24300478 & 18.9900 \\
VCC 2070 & LW2,LW3 & 24101613 & 20.0200 \\
VCC 2087 & LW2 & 24300315 & 19.0800 \\
VCC 2087 & LW3 & 24300447 & 19.0800 \\
VCC 2094 & LW2,LW3 & 24300546 & 19.2400 \\
\hline
\end{tabular}

Galaxies in the clusters A1367 and Coma

\begin{tabular}{llcr}
\hline Target name & Filter & ISO TDT number & Raster orientation \\
$(1)$ & $(2)$ & $(3)$ & $(4)$ \\
\hline 97062 & LW2,LW3 & 22201301 & 19.4100 \\
97073 & LW2,LW3 & 22201102 & 19.4600 \\
97079 & LW2,LW3 & 22201303 & 19.4600 \\
97087 & LW2,LW3 & 22201104 & 19.5100 \\
97138 & LW2,LW3 & 22201606 & 19.6000 \\
160020 & LW2,LW3 & 21501131 & 28.0000 \\
160026 & LW2,LW3 & 21501132 & 28.0300 \\
160055 & LW2 & 61000240 & 13.5000 \\
160055 & LW2,LW3 & 21400934 & 28.8200 \\
160067 & LW2,LW3 & 22803435 & 21.8100 \\
160076 & LW2,LW3 & 21400936 & 29.0400 \\
160086 & LW2,LW3 & 21400937 & 29.0900 \\
160088 & LW2,LW3 & 21500866 & 28.6400 \\
160095 & LW2 & 61700846 & 9.78000 \\
160095 & LW2,LW3 & 21500968 & 28.6700 \\
160095 & LW3 & 61700747 & 9.79001 \\
160127 & LW2,LW3 & 21401169 & 29.5000 \\
160128 & LW2,LW3 & 21401170 & 29.5900 \\
160139 & LW2,LW3 & 21401171 & 29.8800 \\
160252 & LW2,LW3 & 21500865 & 28.6000 \\
160252 & LW3 & 61600443 & 10.2600 \\
160260 & LW2,LW3 & 21500967 & 28.6100 \\
\hline
\end{tabular}

Other galaxies in the Virgo cluster

\begin{tabular}{llcr} 
Other galaxies in the Virgo cluster & & \\
\hline Target name & Filter & ISO TDT number & Raster orientation \\
$(1)$ & $(2)$ & $(3)$ & $(4)$ \\
\hline $1226^{*}$ & LW2,LW3 & 22700815 & 22.5500 \\
1508 & LW2,LW3 & 23600326 & 20.8700 \\
1555 & LW2,LW3 & 23100103 & 21.9800 \\
1632 & LW2,LW3 & 23902129 & 19.2100 \\
$1939^{*}$ & LW2,LW3 & 39600117 & -155.150 \\
CGCG43041 & LW2,LW3 & 22500757 & 23.4300 \\
\hline & & & \\
Other galaxies in the Coma/A1367 supercluster & & & \\
\hline Target name & Filter & ISO TDT number & Raster orientation \\
$(1)$ & $(2)$ & $(3)$ & $(4)$ \\
\hline $159072 \mathrm{~N}, \mathrm{~S}^{*}$ & LW2,LW3 & 24602014 & 9.56000 \\
130023 & LW2,LW3 & 24300658 & 16.6300 \\
\hline
\end{tabular}

Notes:

Galaxies marked with an asterisk have been observed at high resolution with a pixel size of 3 arcsec 
A. Boselli et al.: Mid-IR emission of galaxies in the Virgo cluster. III., Online Material p 13
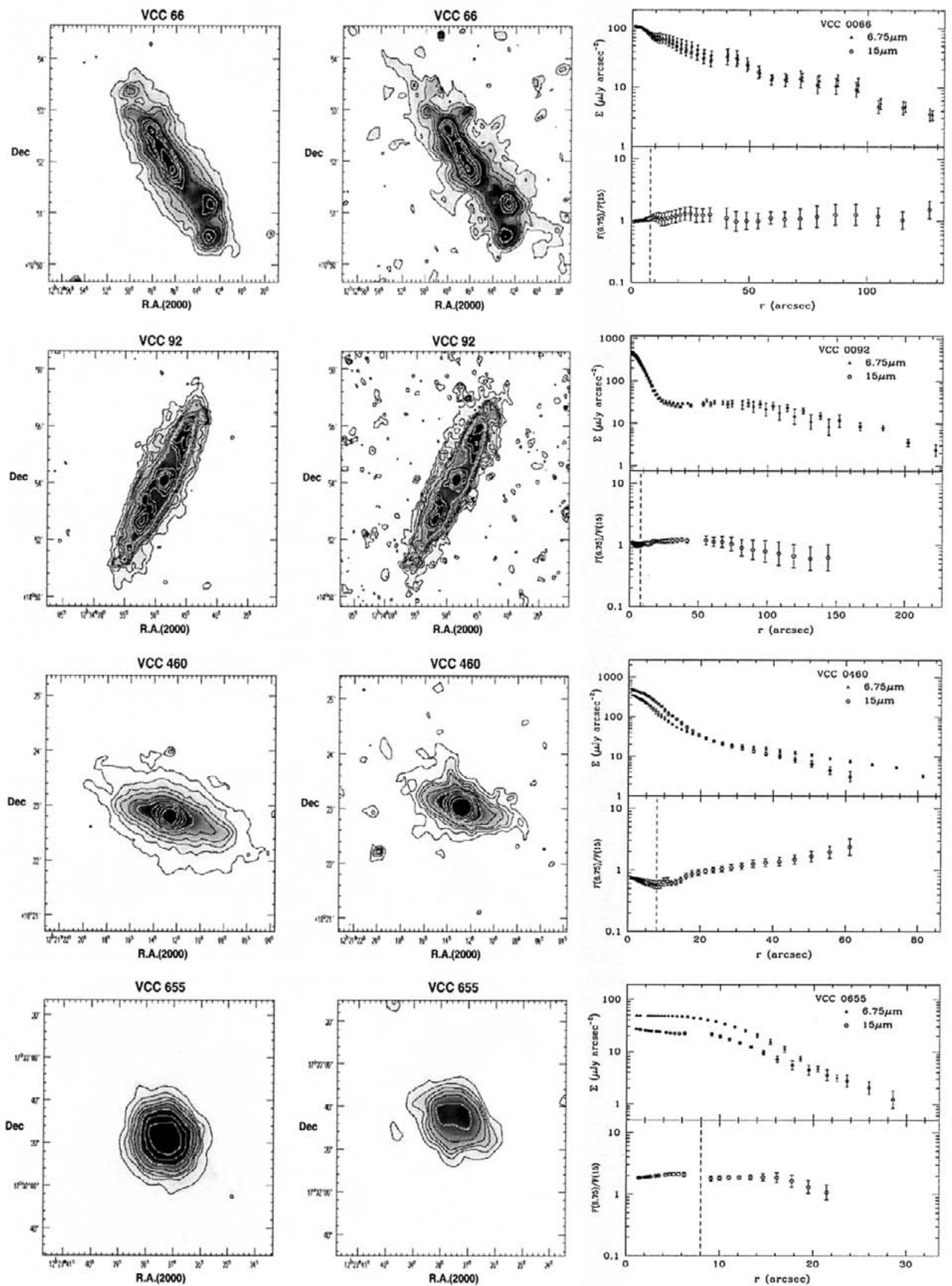

Fig. 1. The gray-scale level images at $6.75 \mu \mathrm{m}$ (left) and $15 \mu \mathrm{m}$ (centre) of the 34 resolved galaxies; contours are at 2, 4, 6, 8, 12 (black), 20, 30, 40, 60, 100 (white) $\mu \mathrm{Jy} \operatorname{arcsec}^{-2}$ (coordinates are $\mathrm{J} 2000$ ). Radial (6.75 and $15 \mu \mathrm{m}$ ) and colour profiles $(6.75 / 15 \mu \mathrm{m}$ ratio) of each galaxy, obtained integrating along elliptical annuli, are given in the right side panel. A vertical dashed line in the colour profile indicates the 8 arcsec diffraction limit of the $15 \mu \mathrm{m}$ image. 
A. Boselli et al.: Mid-IR emission of galaxies in the Virgo cluster. III., Online Material p 14
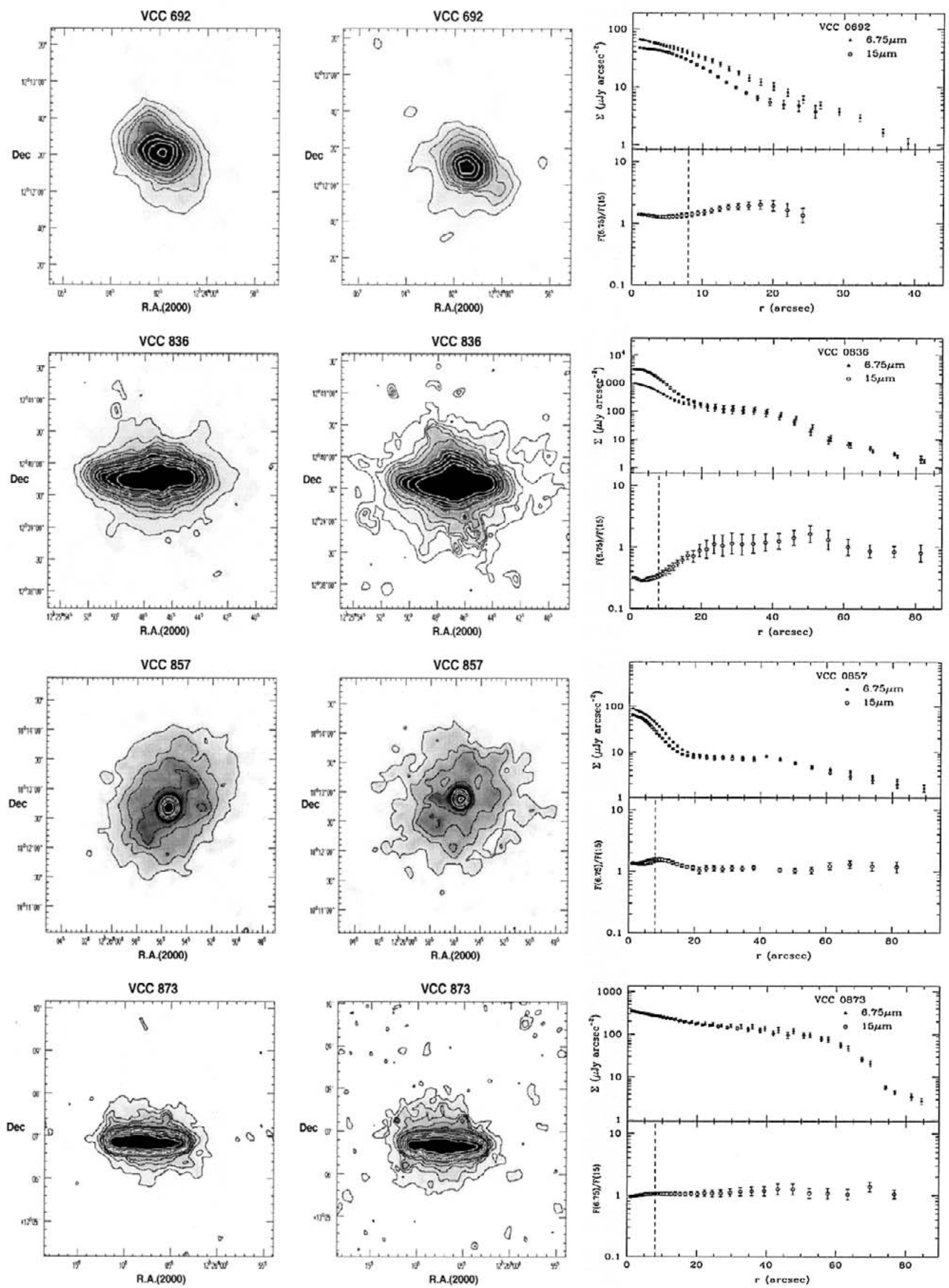

Fig. 1. continued. 
A. Boselli et al.: Mid-IR emission of galaxies in the Virgo cluster. III., Online Material p 15
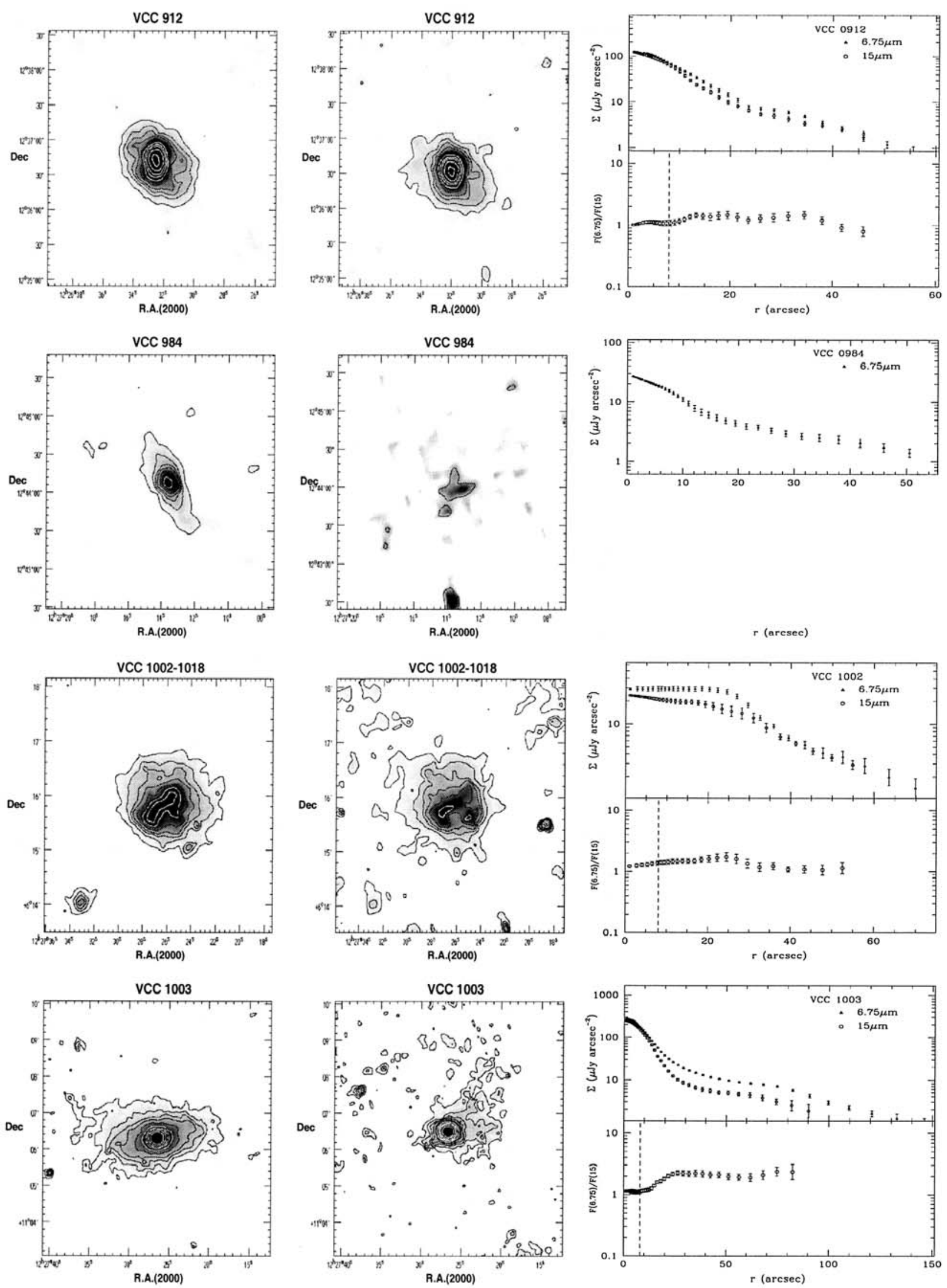

Fig. 1. continued. 
A. Boselli et al.: Mid-IR emission of galaxies in the Virgo cluster. III., Online Material p 16
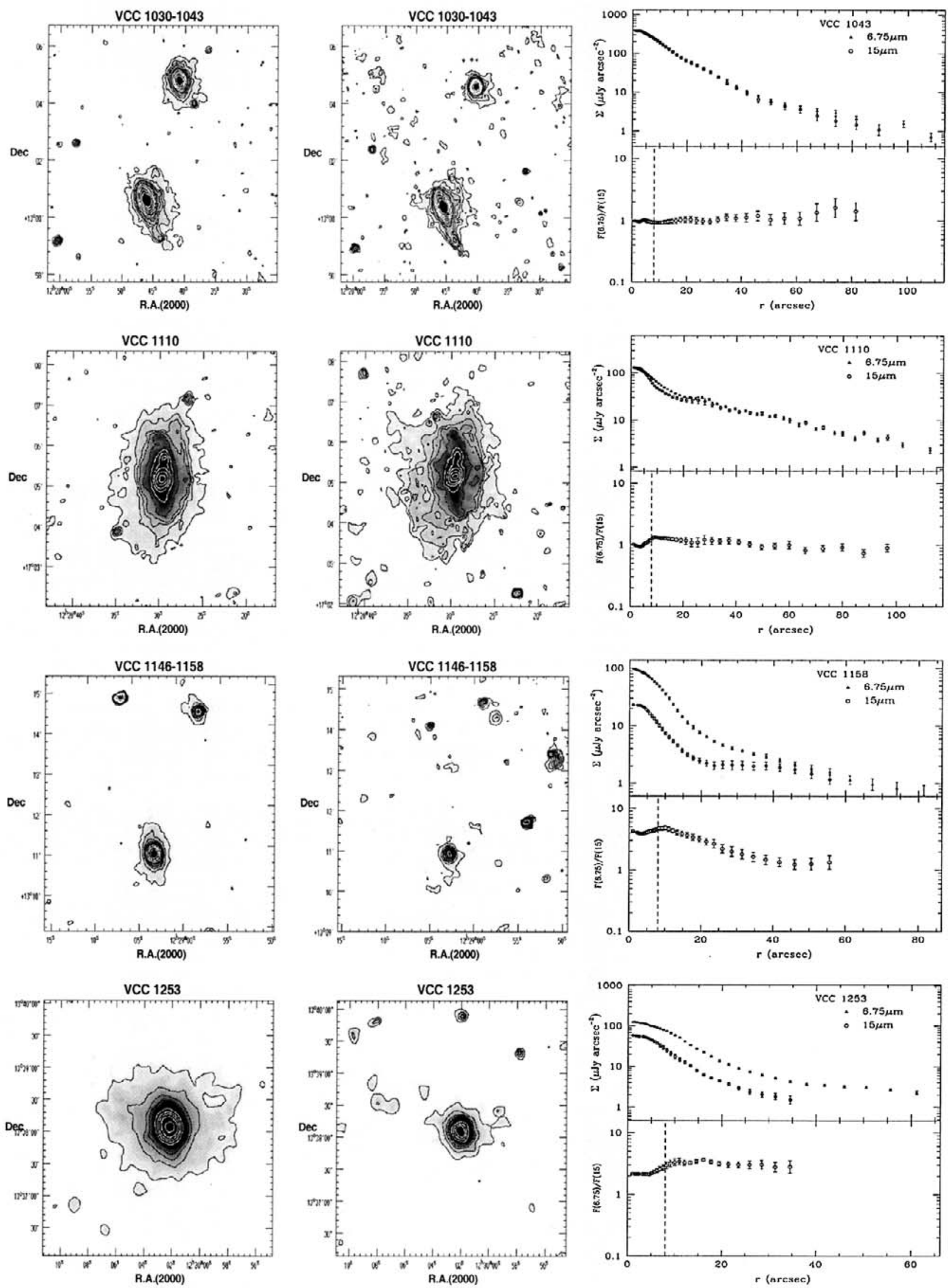

Fig. 1. continued. 
A. Boselli et al.: Mid-IR emission of galaxies in the Virgo cluster. III., Online Material p 17
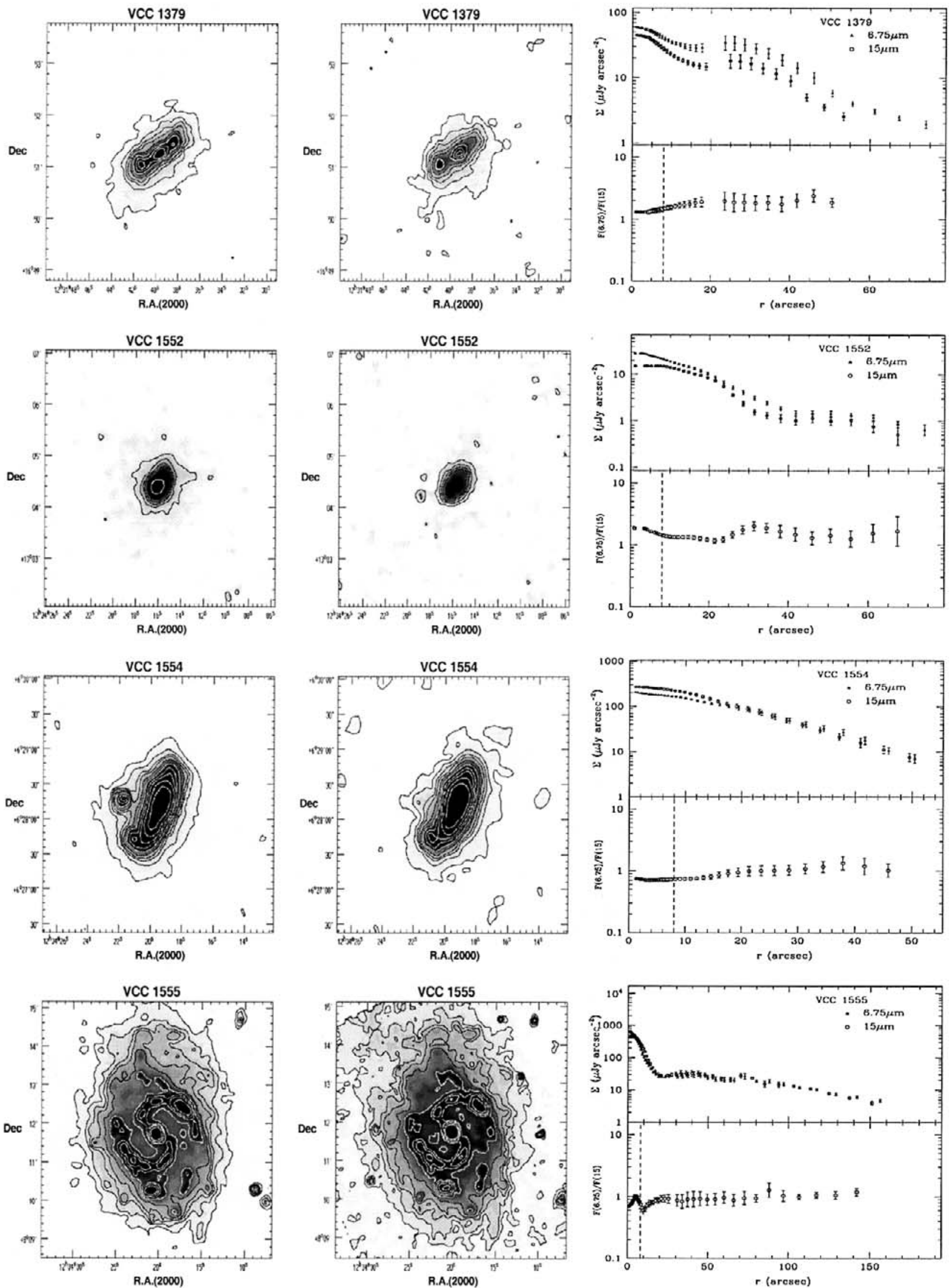

Fig. 1. continued. 
A. Boselli et al.: Mid-IR emission of galaxies in the Virgo cluster. III., Online Material p 18
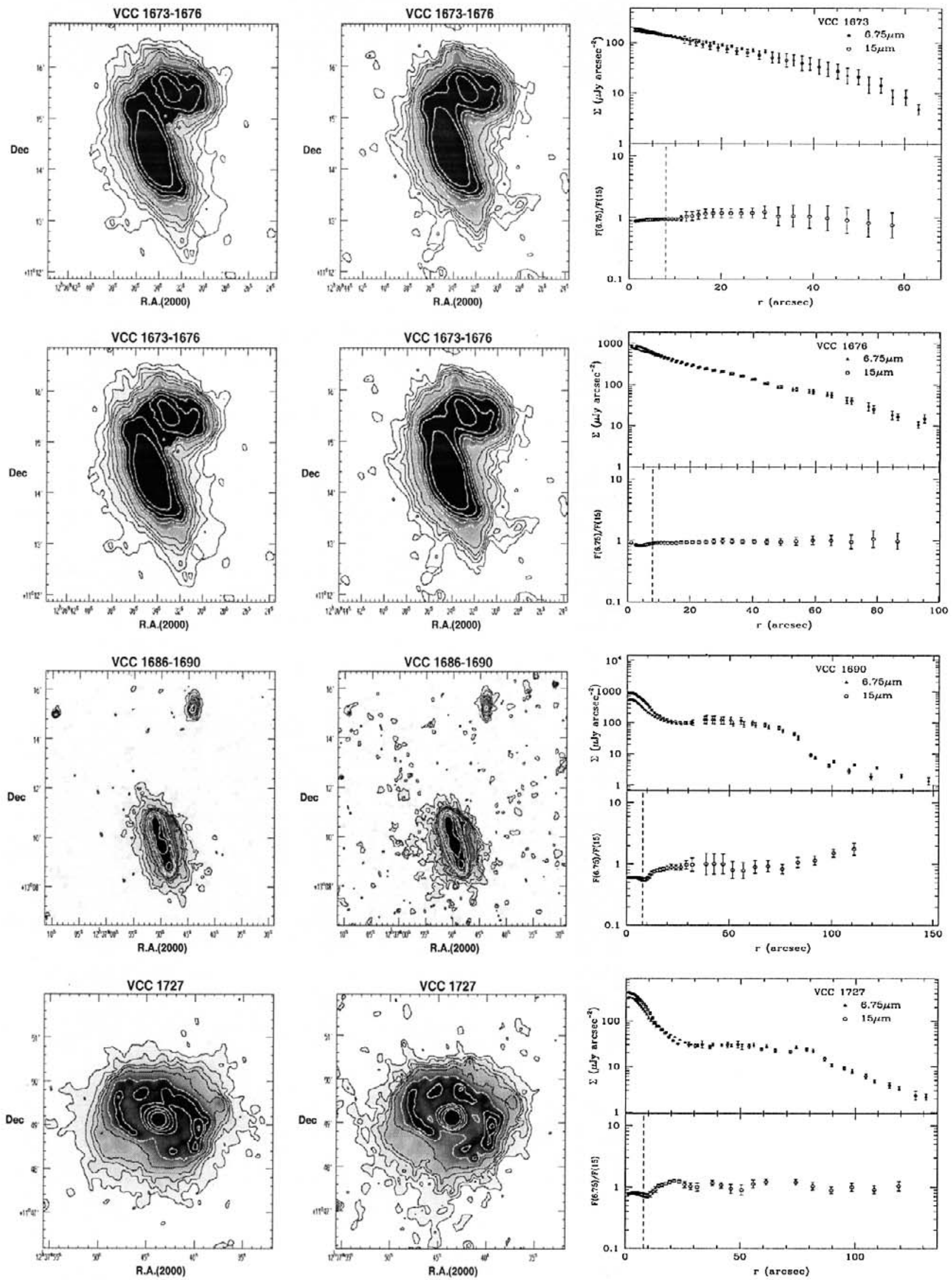

Fig. 1. continued. 
A. Boselli et al.: Mid-IR emission of galaxies in the Virgo cluster. III., Online Material p 19
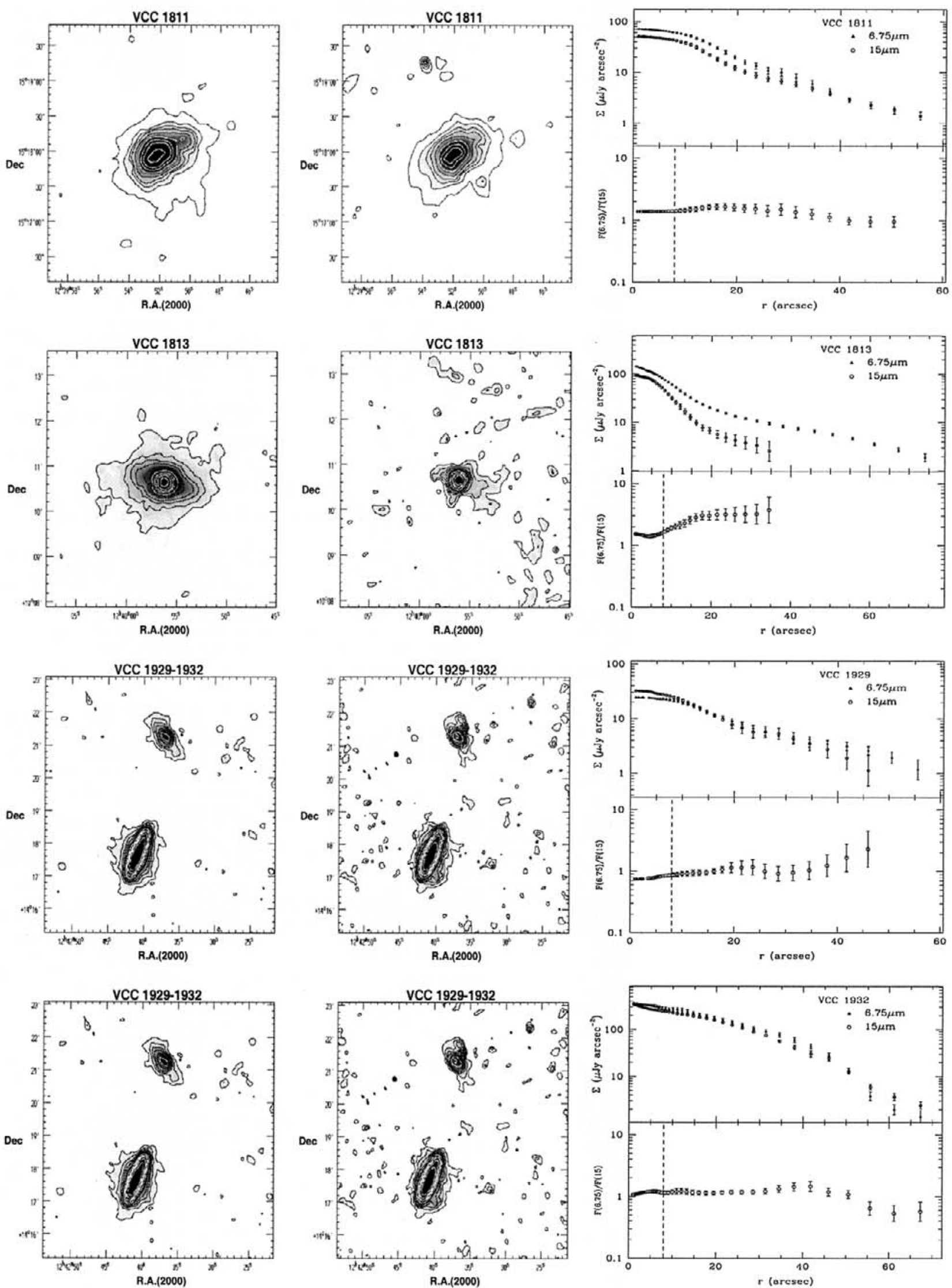

Fig. 1. continued. 
A. Boselli et al.: Mid-IR emission of galaxies in the Virgo cluster. III., Online Material p 20
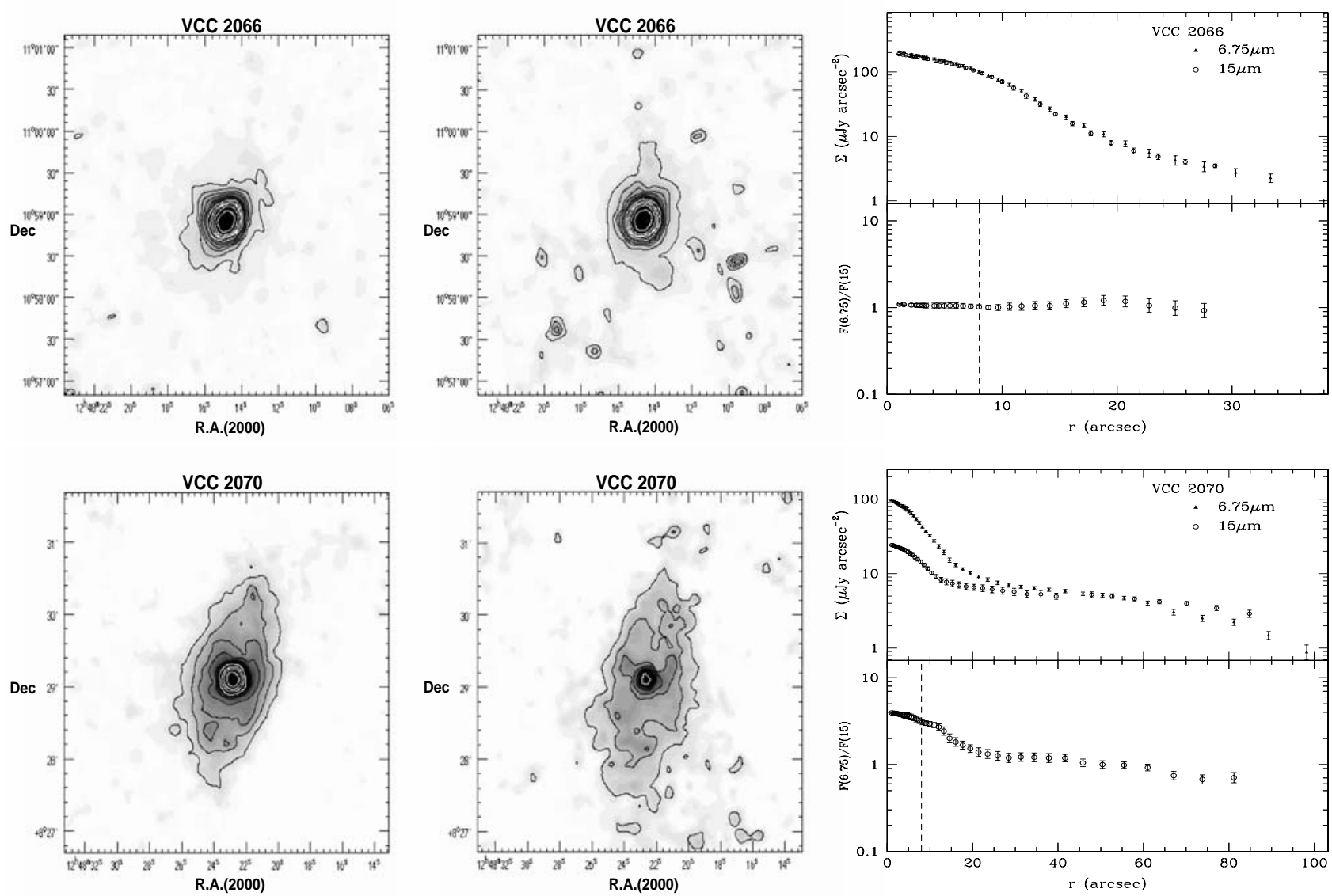

Fig. 1. continued. 
A. Boselli et al.: Mid-IR emission of galaxies in the Virgo cluster. III., Online Material p 21
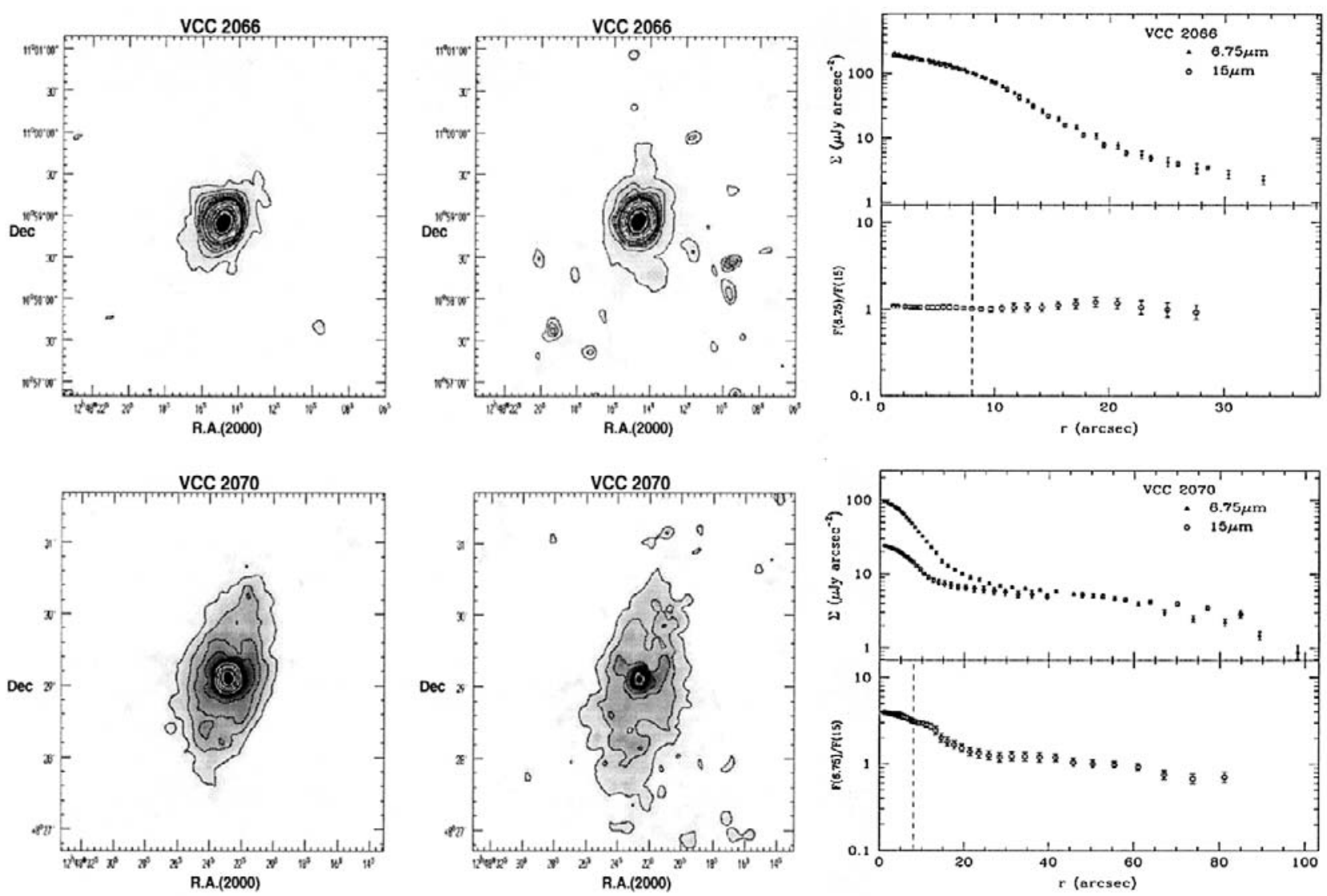

Fig. 1. continued. 
A. Boselli et al.: Mid-IR emission of galaxies in the Virgo cluster. III., Online Material p 22
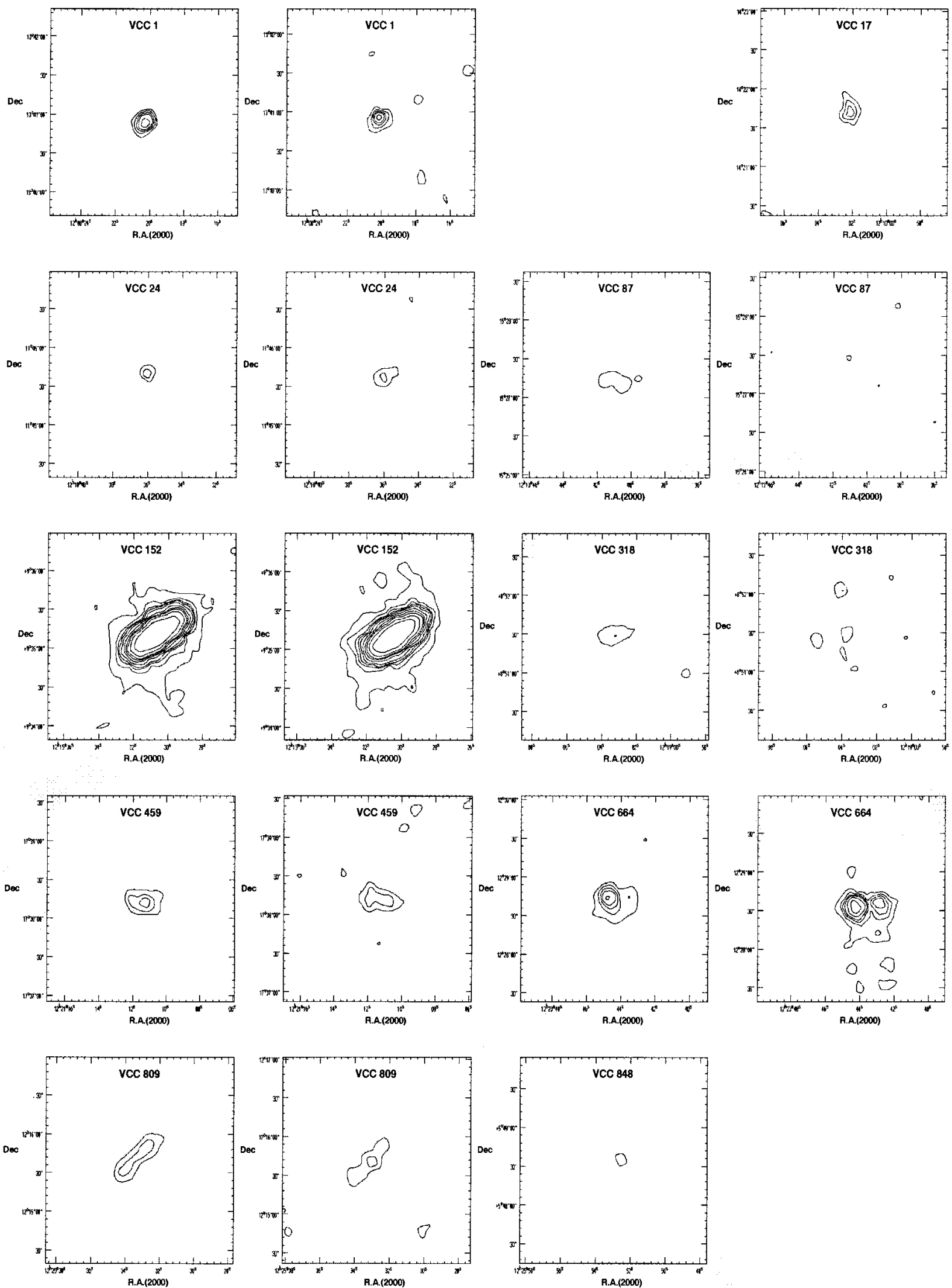

Fig. 2. The contour level images of galaxies detected with a surface brightness brighter than $2 \mu \mathrm{Jy} \operatorname{arcsec}^{-2}$. For each galaxy, the left image is at $6.75 \mu \mathrm{m}$ and the right one at $15 \mu \mathrm{m}$; as for the images of the fully resolved galaxies, contours are at 2, 4, 6, 8, 12, 20, 30, 40, 60, 100 $\mu \mathrm{Jy} \operatorname{arcsec}^{-2}$ (coordinates are $\mathbf{J} 2000$ ). 
A. Boselli et al.: Mid-IR emission of galaxies in the Virgo cluster. III., Online Material p 23
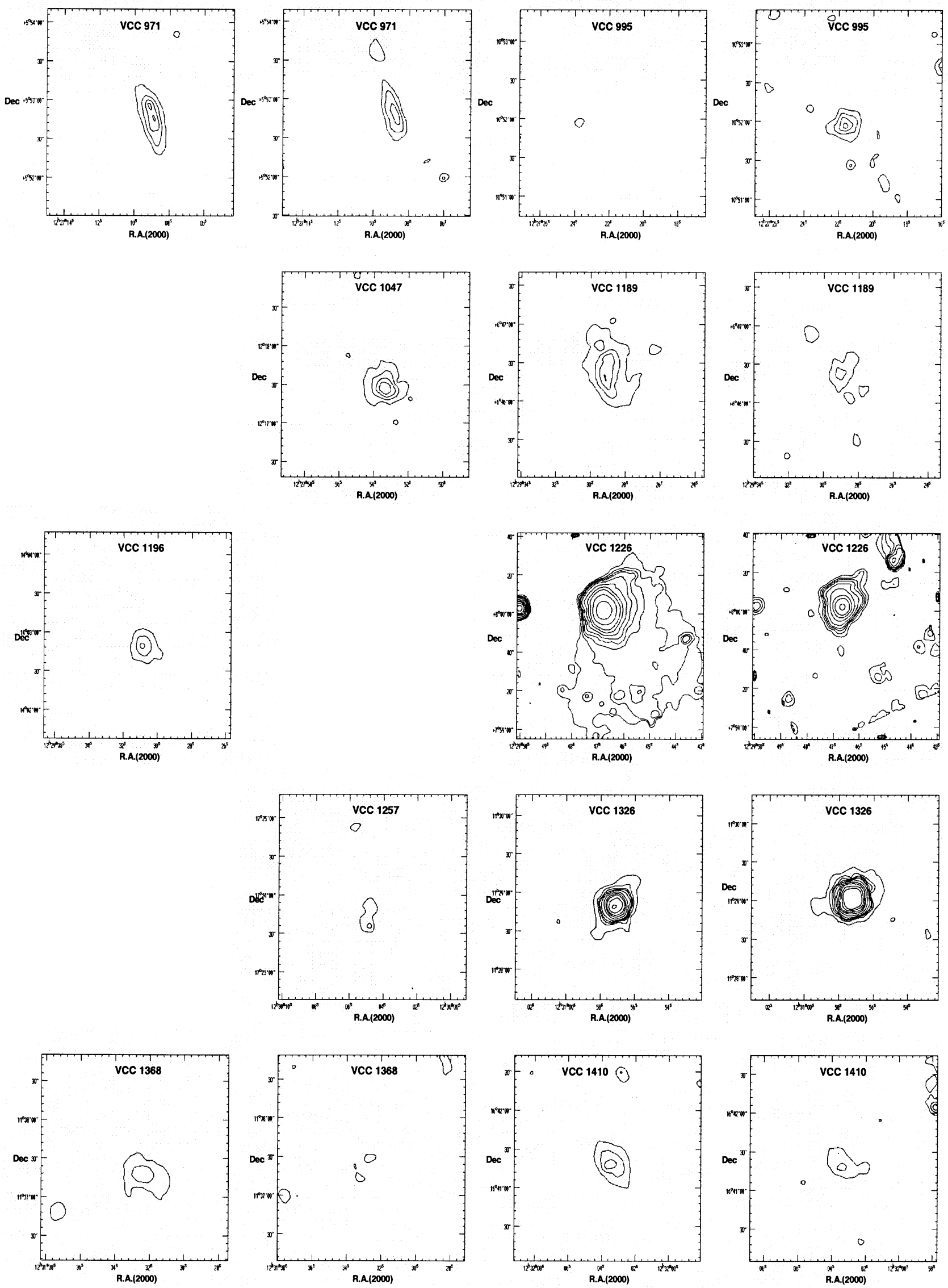

Fig. 2. continued. 
A. Boselli et al.: Mid-IR emission of galaxies in the Virgo cluster. III., Online Material p 24
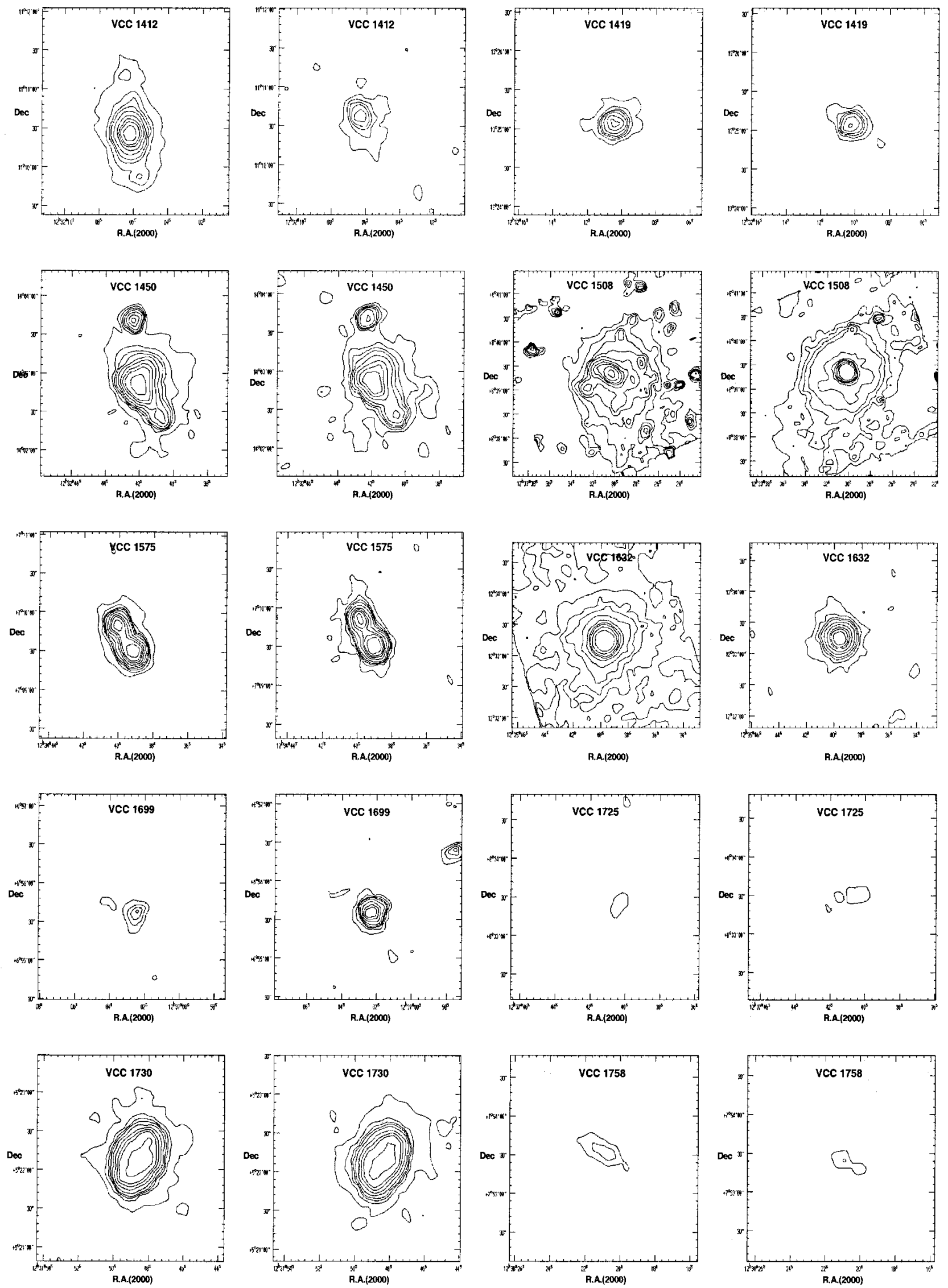

Fig. 2. continued. 
A. Boselli et al.: Mid-IR emission of galaxies in the Virgo cluster. III., Online Material p 25
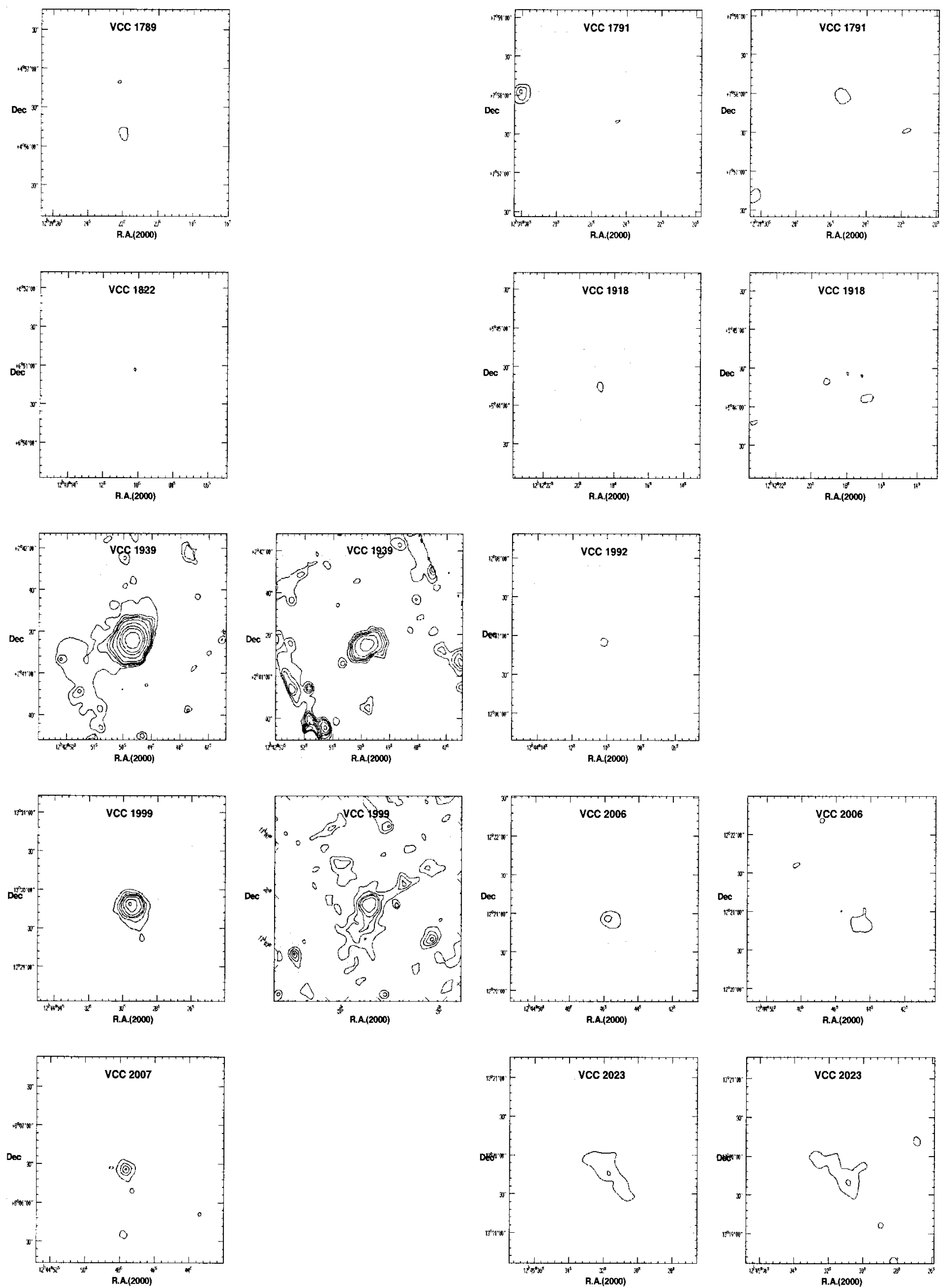

Fig. 2. continued. 
A. Boselli et al.: Mid-IR emission of galaxies in the Virgo cluster. III., Online Material p 26
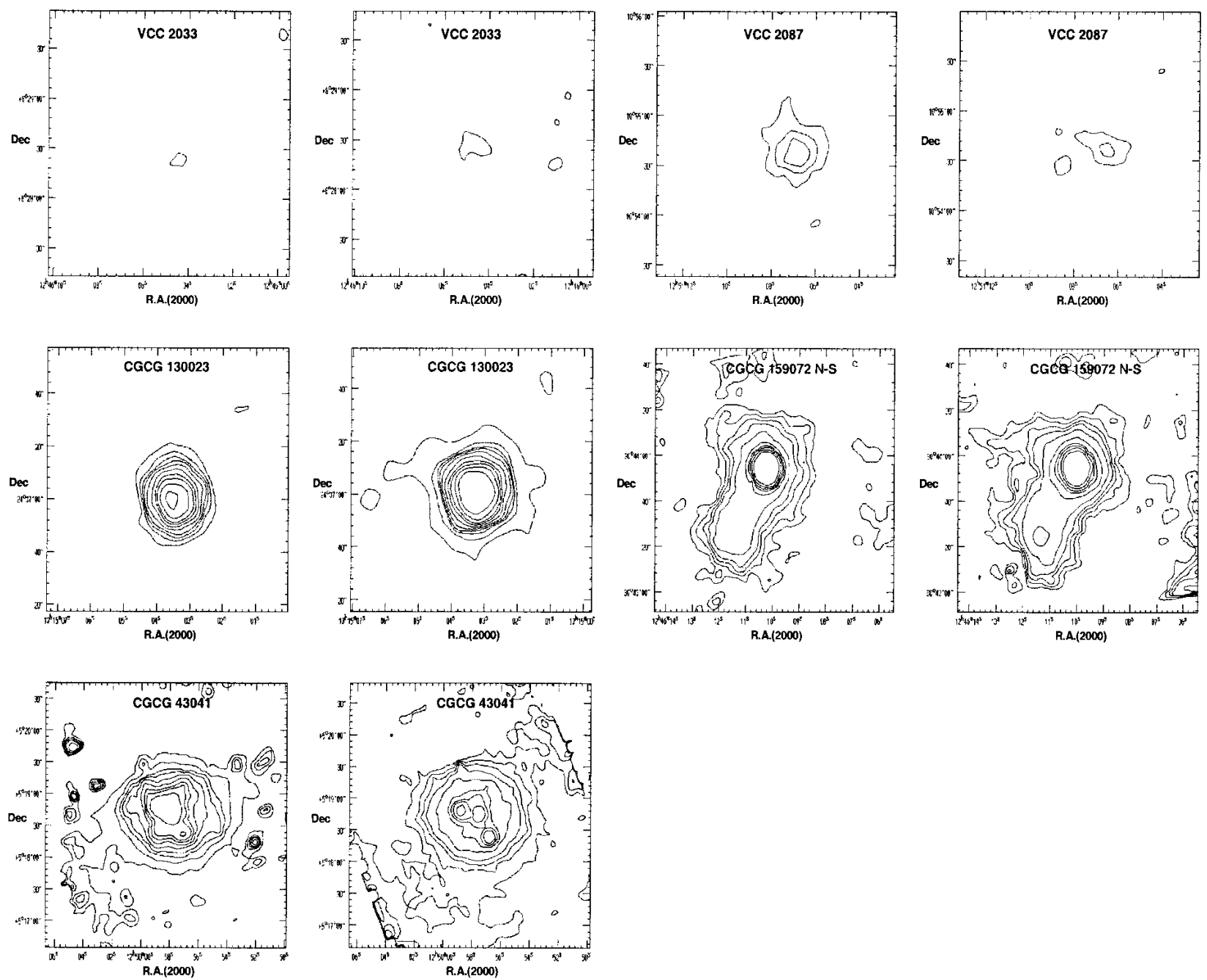

Fig. 2. continued. 